\title{
Platelets in thrombosis and haemostasis
}

Citation for published version (APA):

Li, L. (2022). Platelets in thrombosis and haemostasis: synergy with thrombin generation. [Doctoral Thesis, Maastricht University]. Gildeprint Drukkerijen. https://doi.org/10.26481/dis.20220124II

Document status and date:

Published: 01/01/2022

DOI:

10.26481/dis.20220124\|

Document Version:

Publisher's PDF, also known as Version of record

\section{Please check the document version of this publication:}

- A submitted manuscript is the version of the article upon submission and before peer-review. There can be important differences between the submitted version and the official published version of record.

People interested in the research are advised to contact the author for the final version of the publication, or visit the DOI to the publisher's website.

- The final author version and the galley proof are versions of the publication after peer review.

- The final published version features the final layout of the paper including the volume, issue and page numbers.

Link to publication

\footnotetext{
General rights rights.

- You may freely distribute the URL identifying the publication in the public portal. please follow below link for the End User Agreement:

www.umlib.nl/taverne-license

Take down policy

If you believe that this document breaches copyright please contact us at:

repository@maastrichtuniversity.nl

providing details and we will investigate your claim.
}

Copyright and moral rights for the publications made accessible in the public portal are retained by the authors and/or other copyright owners and it is a condition of accessing publications that users recognise and abide by the legal requirements associated with these

- Users may download and print one copy of any publication from the public portal for the purpose of private study or research.

- You may not further distribute the material or use it for any profit-making activity or commercial gain

If the publication is distributed under the terms of Article $25 \mathrm{fa}$ of the Dutch Copyright Act, indicated by the "Taverne" license above, 


\section{Platelets in thrombosis and haemostasis: synergy with thrombin generation}




\section{D) Maastricht \\ University}
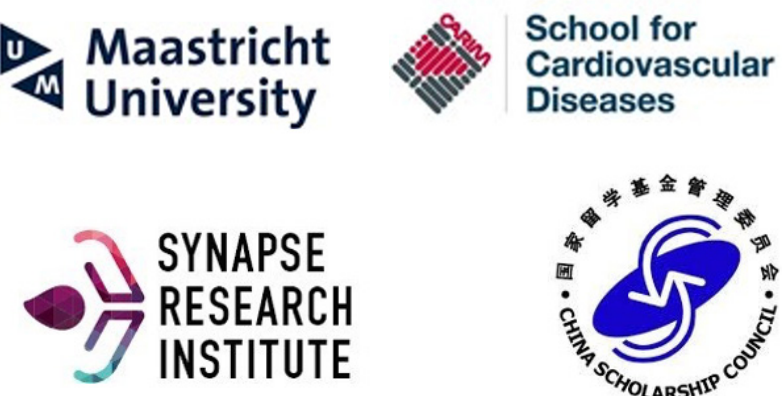

\section{Diseases}

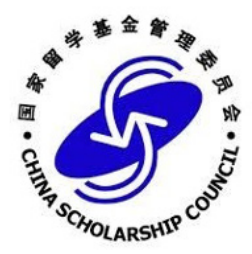

The research in this thesis was supported by an unrestricted grant from Synapse Research Institute and by grants from the China Scholarship Council. The printing of this thesis was supported by Synapse Research Institute.

Cover design: $\quad$ Mingliang Zhu

Lay-out: $\quad$ Ilse Modder | www.ilsemodder.nl

Printed by: Gildeprint Enschede | www.gildeprint.nl

ISBN: $\quad$ 978-94-6419-424-1

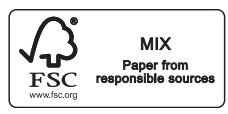

(C) Li Li (2022)

No part of this book may be reproduced or transmitted to any form or by any means without prior permission in writing by the author, or when appropriate, by the publishers of the publications. 


\title{
Platelets in thrombosis and haemostasis: synergy with thrombin generation
}

\author{
Dissertation
}

To obtain the degree of Doctor at Maastricht University, on the authority of the Rector Magnificus, Prof. dr. Rianne M. Letschert, in accordance with the decision of the Board of Deans, to be defended in public on

Monday 24th January 2022 at 13:00 hours

by

Li Li 


\section{Promotor}

Prof. dr. Hugo ten Cate

\section{Co-promotors}

Dr. Dana Huskens

Dr. Mark Roest

\section{Assessment committee}

Prof. dr. Yvonne Henskens, chair

Prof. em. Johan Heemskerk

Dr. Rory Koenen

Prof. dr. Ton Lisman (Groningen)

Prof. dr. Monique Minnema (UMC Utrecht) 


\section{CONTENTS}

Chapter $1 \quad$ General introduction and outline

Chapter 2 Platelet activation via glycoprotein VI initiates thrombin generation: a potential role for platelet-derived factor IX?

Chapter 3 Patients with multiple myeloma have a disbalanced whole blood thrombin generation profile

Chapter 4 Acute exacerbations of COPD are associated with a 65 prothrombotic state through platelet-monocyte complexes, endothelial activation and increased thrombin generation

Chapter $5 \quad$ Flow cytometric analysis of platelet function to detect high on-treatment residual platelet reactivity in patients on dual antiplatelet therapy

Chapter $6 \quad$ Flow cytometric analysis of platelet function to improve 95 the recognition of thrombocytopathy

Chapter $7 \quad$ Analytical characterization and reference interval of an enzyme-linked immunosorbent assay for active von Willebrand factor

Chapter $8 \quad$ Summary, discussion and prospects 



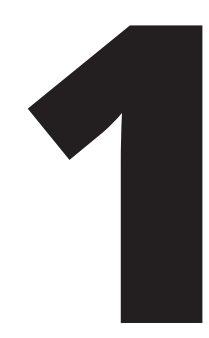

General introduction and outline 


\section{GENERAL INTRODUCTION}

\section{Haemostasis}

The hemostatic reaction to vascular injury requires a complex and highly regulated mechanism that involves platelets, red blood cells, (anti-)coagulation proteins, and vascular wall components (endothelial cells and von Willebrand factor (VWF)) to form a localized hemostatic plug that prevents bleeding [1].

\section{The role of platelets in haemostasis}

Resting circulating platelets have a discoid shape and do not interact with the intact vessel wall. When the vessel wall is damaged, the reaction of platelets can be roughly dissected into adhesion, activation and aggregation (Figure 1).

Under physiological conditions, circulating VWF is organized in a compact globular conformation and does not spontaneously interact with platelets [2, 3]. Immobilization of VWF to exposed subendothelial collagen or elevated shear stress (as in stenosis) results in the unfolding of VWF and in the expose of its A1 domain allowing platelets to transiently bind via GPIba, to slow down, and to further bind to matrix proteins via other receptors like a2ß1 and GPVI, all leading to a stable platelet adhesion [4-6].

Platelet adhesion allows collagen GPVI interaction, and hence platelet activation. This platelet activation results in cytoskeletal rearrangements (shape change) and exposure of phosphatidylserine (PS) at platelet surface, platelet granule release and integrin activation $[7,8]$.

Platelet secretion is characterized by the release of a-granule proteins including membrane bound receptors (P-selectin, CD63 and CD40L), coagulation related factors (including factor (F)V, FVIII, protein S, antithrombin (AT), plasminogen/plasmin, plasminogen activator inhibitor 1 (PAI-1), C1-inhibitor, nexin 1 and 2, cytokines, chemokines, growth factors, microbicidal proteins and immune mediators), and secretion of dense granules containing high concentrations of adenine nucleotides (ADP and ATP), serotonin, histamine, $\mathrm{Ca}^{2+}, \mathrm{Mg}^{2+}, \mathrm{K}^{+}$, pyrophosphate and polyphosphate (PolyP). The secreted ADP together with locally generated thromboxane $\mathrm{A}_{2}$ and traces of thrombin induce the secondary platelet activation response via the $P 2 Y_{1} / P 2 Y_{12}$, thromboxane (TP) and PAR-1/PAR-4 receptors, respectively, evoking an amplification of platelet activation [9-12].

In addition to granule release, integrin allbß3 activation is another characteristic of platelet activation. Active allb $\beta 3$ binds to fibrinogen, which is bound to another platelet to form platelet-fibrinogen bridges. Multiple platelet fibrinogen complexes will lead to platelet aggregates formation [7]. At low shear rate, platelet aggregation is 
mainly mediated by allbß3-fibrinogen interactions, with soluble agonists maintaining the activated state of the integrins. At higher shear rate, platelet-platelet interactions become increasingly dependent on VWF and its binding to GPIba receptors [13-16] .

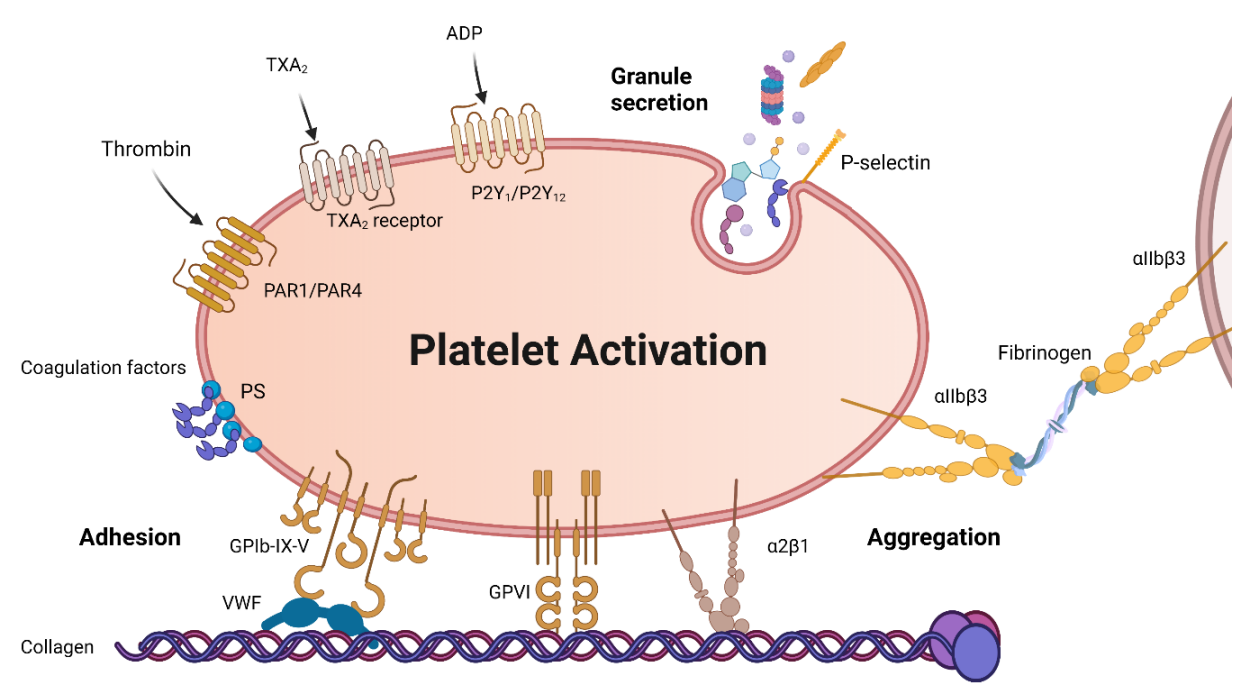

Figure 1. Platelet's reactions during hemostasis, see above paragraph for detailed explanations, illustration created with Biorender.com.

\section{Coagulation}

In venous blood, the shear rates are lower than in arteries. At low shear rates the enzymatic coagulation process is the most important pathway in haemostasis and thrombosis. The physiological coagulation pathway is triggered by tissue factor (TF) exposed at sites of vascular injury. TF binds FVIIa, which is present in the blood. The TFFVIla complex activates FIX and FX into FIXa and FXa, respectively. FXa can activate more FVII, accelerating the start of the coagulation. During initiation of coagulation, FXa alone can cleave prothrombin to produce trace amounts of thrombin, and this thrombin can activate coagulation factors FV, FVIII and FXI. Thrombin is the central player of coagulation, that converts fibrinogen to fibrin monomers, which polymerizes and are cross-linked into a dense clot to seal the wound. In addition to TF, coagulation can be triggered by the contact of FXII with negatively charged surfaces, such as polyphosphates (polyP), double stranded DNA or misfolded proteins. Active FXII activates FXI and FIX, which further activate FX and prothrombin.

To prevent excessive thrombin formation, there are several natural anticoagulant proteins present in the circulation, including tissue factor pathway inhibitor (TFPI) which 
inhibits FVIla and FXa; antithrombin and alpha2-macroglobulin that target FIXa, FXa and thrombin. Also thrombin binds to thrombomodulin (TM) on endothelial cells and activates protein $\mathrm{C}$, which then, together with its cofactor protein S, degrades FVIIIa and FVa to limit thrombin generation [17].

\section{Fibrinolysis}

Besides limiting clot growth, degradation of the fibrin clot (fibrinolysis), is crucial to maintain a balance in haemostasis. Plasmin is the key protease of the fibrinolytic system, cleaving fibrin into fibrin degradation products (FDPs). During clot development, plasmin is generated from plasminogen, which is mediated by tissue-type plasminogen activator (tPA), released from endothelial cells, and to a lesser extent, by urokinase-type plasminogen activator (UPA) [18].

\section{Platelets' participation in coagulation}

The interplay between platelets and coagulation is more effective than the two processes separately $[19,20]$ (Figure 2). At the initial stage of coagulation activation, platelets are activated by thrombin via the PAR- 1 and PAR-4 receptors and via the GPIbIX-V complex, resulting in a strong platelet activation, exposure of phosphatidylserine (PS) and amplification of coagulation, respectively. The PS on the platelet membrane can act as assembly sites for the tenase complex (FIXa together with FVIIla activating FX) and the prothrombinase complex (FXa together with FVa activating prothrombin), increasing the activities of these complexes by nearly 1000-fold, resulting in a burst of thrombin in the propagation phase of coagulation [21-23]. Furthermore, activated platelets supply partially activated FV from a granules to support the coagulation cascade and PolyP from dense granules that may cause FXII activation and promotion of FXI back-activation by thrombin [24-27].

\section{Platelets and inflammation}

Apart from its traditionally well-known role in hemostasis, there is growing recognition that platelets also have a critical role in inflammation and immune responses [28]. Platelets can directly or indirectly interact with bacteria via allb33 or GPIba, leading to platelet activation and aggregation [29-32]. During activation, platelets secrete a wide range of mediators of inflammation, including P-selectin, CD40L, platelet factor 4, macrophage inflammatory protein (MIP)-1a, interleukin (IL)-1 and IL-8 that have predominantly proinflammatory effects [28]. Especially, platelet P-selectin expression and subsequent formation of platelet-leukocyte aggregates upregulates leukocyte pro-inflammatory functions [33]. Furthermore, platelet TLR4 can interact with bacterial lipopolysaccharide (LPS), increasing platelet adhesion to fibrinogen and inducing the formation of neutrophil extracellular traps (NETs) that ensnare bacteria [34, 35]. Taken together, activated platelets may serve as a link for hemostatic and inflammatory responses. 


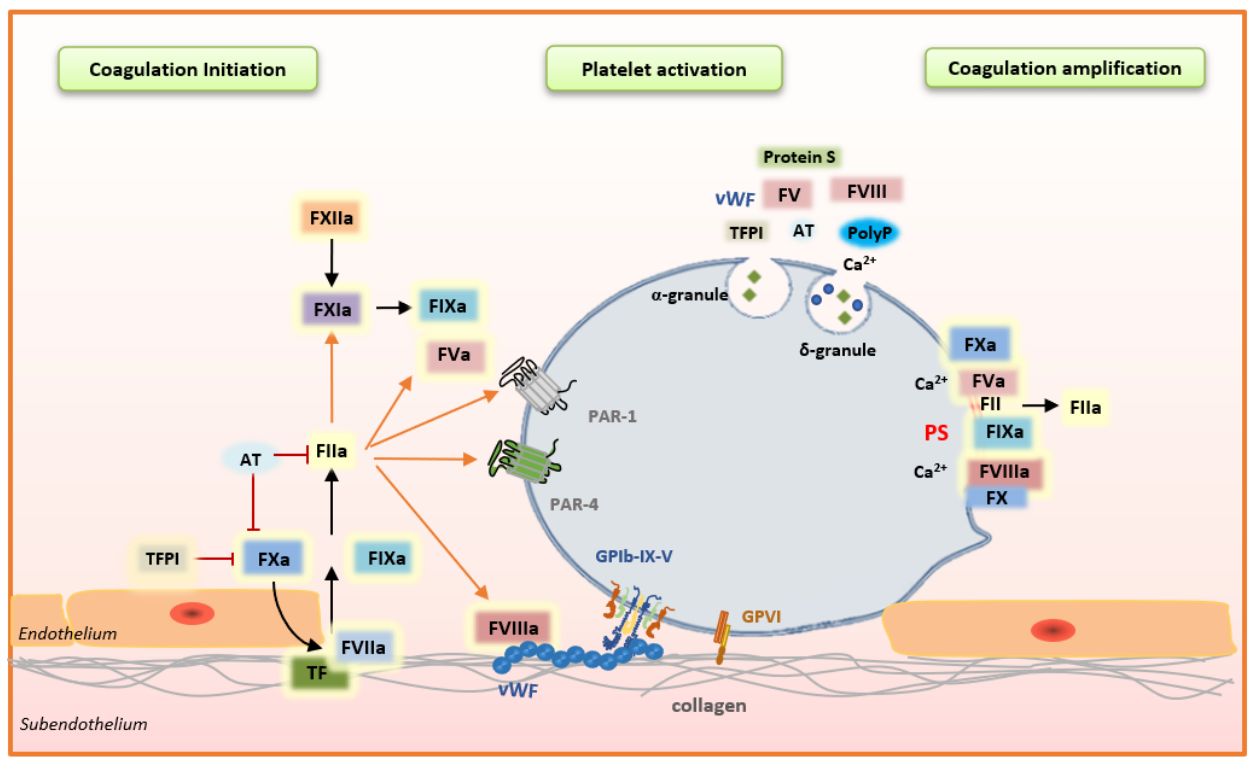

Figure 2. Coagulation and platelet-based amplification of coagulation, adapted from Sang et al., Blood reviews 2021, 46:100733.

\section{Platelet function abnormalities in diseases}

Since the essential role of platelets in the maintenance of normal haemostasis, (congenital) defects in platelet reactivity can lead to easy or spontaneous bruising, mucocutaneous bleeds, or prolonged bleeding [36-38]. Platelet defects can be caused by disturbed intracellular signaling pathways (thromboxane A synthase (Ghosal syndrome ) and cytosolic phospholipase A2), by the presence and function of platelet receptors (defective or low levels of allbß3 (Glanzmann's thrombasthenia), deficiency of GPIb-IX-V (Bernard-Soulier syndrome), GPVI deficiency, P2Y 12 receptor deficiency), in granule secretion (alpha granule formation defect (grey platelet syndrome) and dense granule deficiency (Hermansky-Pudlack and Chediak-Higashi syndrome)), or by the defective scrambling of phospholipids on platelets (Scott syndrome).

\section{Platelet function (reactivity) tests}

Several platelet reactivity assays have been developed to assist in the diagnosis of platelet related disorders of haemostasis and to assess anti-platelet therapy [39, 40]. Tests to measure platelet aggregation under static conditions are light transmission aggregometry (LTA), VerifyNow and PlateletWorks. Furthermore, the Multiplate (impedance-based aggregometry) measures aggregation under stirring conditions while the cone and platelet analyzer (CPA) and platelet function analyzer (PFA-100/PFA200) measure aggregation in the presence of shear forces [41]. Tests to detect platelet 
secretion defects focus on measuring the ATP release from dense granules (luminometry test) or on assessing granule number and function (transmission electron microscopy (TEM)/immunofluorescence assays). Furthermore, there are microfluidics/flow chamber assays that assess platelet deposition and thrombus growth on coated surfaces under flow by microscopy and there are flow cytometric assays analysing the expression of activation markers on platelet surfaces with fluorescence-labelled antibodies.

For the diagnosis of bleeding disorders, LTA is the most commonly used and is therefore considered as the gold standard in clinical practice [42]. The technique determines platelet aggregation percentage in platelet-rich plasma (PRP) by measuring the increase in light transmission in response to the addition of a platelet agonist. However, LTA has several limitations as it is not very sensitive for mild platelet function disorders and it is quite laborious, time consuming and requires large volumes of blood and a minimal platelet count of $150 * 10^{9} / L[42,43]$.

The use of flow cytometry has been recommended as an integral component of the investigation of platelet function disorders under several guidelines [37, 44]. The method of detecting activated platelets in whole blood by using activation-dependent monoclonal antibodies and flow cytometry was first described in 1987 by Shattil et al [45]. Similar to the agonist panel in LTA, multiple agonists (e.g. PAR-1 activating peptides like thrombin receptor activating peptide-6 (TRAP-6), PAR-4 activating peptides, ADP, crosslinked collagen-related peptide (CRP-XL) or convulxin, arachidonic acid or thromboxane A2 mimetic) can be used to assess the common platelet signaling pathways leading to platelet activation. Platelet reactivity can be determined by measuring platelet activation markers such as a-granule membrane markers (P-selectin, CD40L), dense granule membrane marker (CD63) or by detection of activated integrin, allb $\beta 3$ (antibody PAC-1 or the binding of fluorochrome-conjugated fibrinogen). Besides the classical markers of platelet activation, flow cytometry can be used to assess the expression of anionic phospholipids on activated platelets for testing platelet procoagulant activity (fluorochrome-conjugated annexin-V or lactadherin). Also, VWF-binding (anti-VWF antibody staining) can be indicative of VWF-mediated platelet agglutination and biallelic Bernard-Soulier syndrome, platelet type VWD and acquired VWD $[46,47]$.

The flow cytometric measurement of platelet reactivity has several advantages. The single cell-based analysis makes the test largely independent of platelet count, therefore it is feasible to measure platelet function in blood from patients with severe thrombocytopenia. However, a platelet count lower than $10 \times 10^{9} / \mathrm{mL}$ might influence in vitro platelet activation due to the decreased release of ADP, which serves as important amplifier of platelet activation [48]. Furthermore, only small amounts of whole blood are required making the technique suitable for measuring platelet response in neonatal 
samples. However, at this moment, protocols to measure platelet reactivity with flow cytometry still vary considerably between different laboratories, and each laboratory needs to establish intra-laboratory standardization, validation and determination of reference ranges.

\section{In vivo platelet activation evaluated by platelet-monocyte complexes (PMCs)}

Although P-selectin is considered as the 'gold standard' marker of platelet activation, in vivo circulating degranulated platelets rapidly lose their surface P-selectin, decreasing the sensitivity of P-selectin measurement as platelet activation status evaluation [49]. Besides measuring P-selectin expression, there is increasing interest in measuring platelet-monocyte complexes. Upon activation, platelets can form complexes with monocytes via the binding between P-selectin and P-selectin glycoprotein ligand-1 (PSGL-1), which is constitutively expressed on leukocyte surfaces. The complexes are further stabilized by the binding of platelet GPIba and monocyte macrophage-1 antigen (Mac-1) [50]. Circulating PMCs can be quantified with flow cytometry as the number of events double positive for platelet markers and monocyte markers. There has been some research suggesting that other than P-selectin, PMCs may have the potential to be a marker for measuring in vivo platelet activation [51] and increased PMCs were found in stable coronary artery disease, unstable angina and acute myocardial infarction [52-56].

\section{Clinical tests to measure coagulation}

The two classic clotting-based methods, prothrombin time (PT) and activated partial thromboplastin time (APTT), are the most used first line screening to test bleeding phenotype [57]. Both tests measure the time taken for a plasma sample to form a dense fibrin clot after samples are triggered with either an extrinsic or intrinsic activator (kaolin). Besides classical endpoint assays, there are viscoelastic assays and thrombin generation (TG) assays measuring the kinetics of fibrin or thrombin formation, respectively. The viscoelastic assays utilize mechanical rotation to detect the dynamics of clot formation, stabilization and dissolution in whole blood sample, thus providing the time of clot formation and also information on platelet function and fibrinolysis [58]. TG assays, for instance the Calibrated Automated Thrombography (CAT), measure the concentrations of thrombin formed over time by monitoring the cleavage of a thrombin substrate [59, 60]. Studies have shown that TG assays can be used to elucidate coagulation mechanisms, to investigate haemorrhagic coagulopathies, to monitor antithrombotic drugs and to predict risk of venous thromboembolism [59, 61].

Many efforts have been made to develop assays that can measure thrombin formation in whole blood and recently, with some optimizations that prevent erythrocyte sedimentation during measurement, a novel whole-blood thrombin generation (WBTG) assay was developed [62]. WB-TG is one step closer to physiology by involving the 
intrinsic blood cells instead of synthetic phospholipids. Furthermore, because recent findings have suggested that abnormal blood cell characteristics may contribute to coagulopathies, this assay may be a solution for investigating the prothrombotic role of blood cells in thrombotic disorders.

\section{OUTLINE OF THE THESIS}

The first part of this thesis is to provide some insights into the interplay between platelets and coagulation, with 2 studies investigating platelet activation and coagulation profiles in patients who have increased thrombosis risk.

The complex interplay between platelets and the coagulation system has been underestimated for decades. Although the awareness that many steps in platelet thrombus formation are closely connected to the different stages of thrombin formation, little is known about the mechanisms behind it. For example, collagen can not only trigger coagulation via activation of FXII, it can also trigger coagulation independently of FXII. In chapter 2 we explored a novel mechanism of coagulation initiation via collagendependent platelet activation in vitro. To investigate this mechanism in more detail, we used the CAT method, and TG was initiated with the GPVI-specific agonists collagenrelated peptide (CRP-XL, cross-linked peptide composed of GPO repeats) and convulxin (venom of the snake Crotalus durissus terrificus) that bind selectively to GPVI and not to the integrin a2 $\beta 1[63,64]$.

Although the interplay between platelets and the coagulation system is important, and many disease- and therapy-related thrombotic events are induced by damaged or affected platelets rather than by changes in coagulation factors, there are still no accessible tools to study this in clinical research. This may explain the lack of associations between plasma coagulation tests and thrombotic incidents. Viscoelastic tests, such as ROTEM and TEG are used to study blood cell-mediated thrombotic risk, however, these tests are not specific to be a serious alternative for plasma coagulation tests or platelet function tests. Our group recently developed a whole blood thrombin generation test (WB-TG) that shows good correlation with plasma thrombin generation and this test was used in chapter 3 to investigate the whole blood coagulation profile in multiple myeloma (MM) patients and healthy controls. Multiple myeloma is associated with an increased incidence of venous thromboembolism (VTE), despite a high prevalence of thrombocytopenia and anaemia. The real cause of the high VTE risk remains unclear, despite several studies that have been performed on the relation between global coagulation and VTE risk. In this study, platelet function, WB-TG and plasma TG were measured to investigate the paradoxical high prevalence of bleeding symptoms versus 
an increased thrombosis risk.

In chapter 4, the prothrombotic state in chronic obstructive pulmonary disease (COPD) patients following acute exacerbation was investigated. Exacerbations are associated with increased systemic inflammation, which may drive coagulation. In this prospective cohort study, we determined how acute exacerbations in COPD affects platelet activation, the endothelium, plasmatic coagulation and fibrinolysis. We also investigated the association with inflammation as the prevalence of thrombotic events is known to increase during and shortly after acute exacerbations of COPD, but the precise mechanism is unknown.

In a second part of this thesis, we focus on the whole blood flow cytometric platelet activation test (WB-PACT). In chapter 5, platelet function was measured in patients on dual anti-platelet therapy. Dual antiplatelet therapy (DAPT) with aspirin and the P2Y receptor antagonist is commonly prescribed as secondary prevention for patients after percutaneous angioplasty with stent implantation, and it was shown to effectively reduce future ischemic events in patients with atherosclerotic cardiovascular disease. Several studies indicated high on-treatment residual platelet reactivity (HRPR) in patients on DAPT with higher risk of thrombotic events. In this study platelet function triggered with ADP was measured with the WB-PACT and compared with the golden standard LTA to detect high on treatment residual platelet reactivity. In chapter 6, a population of patients with suspected thrombocytopathy were tested with WB-PACT. Although LTA is the 'gold standard' platelet function test to define suspected bleeding disorders, it still lacks sensitivity for mild platelet function defects, and it does not predict the risk of bleeding complication among patients with various platelet defects. Therefore, there is an urgent need for alternative methods to give complimentary information about mild platelet function defects. In this study, both the WB-PACT and LTA were conducted using multiple agonists to determine platelet function. Furthermore, the VWF function application of the WB-PACT was compared with the ristocetin induced agglutination measurement for detecting VWF disfunction. Both tests use ristocetin to activate VWF, however, this does not reflect the in vivo situation. In Chapter 7, an immunosorbent assay was characterized to directly detect circulating VWF in its active conformation. This assay is based on a recombinant llama-derived antibody directed against a cryptic epitope in the A1 domain of VWF. The aims of the current study were to (1) characterize the analytical performance of this assay; (2) provide a reference interval for active VWF and (3) determine correlations between this assay and other, established VWF assays and a whole blood flow cytometric assay for platelet-VWF binding.

Chapter 8 provides a summary of findings in this thesis and possible future research directions. 


\section{REFERENCES}

1. Ivanciu L, Stalker TJ: Spatiotemporal regulation of coagulation and platelet activation during the hemostatic response in vivo. Journal of thrombosis and haemostasis : JTH 2015, 13(11):1949-1959.

2. Slayter H, Loscalzo J, Bockenstedt P, Handin Rl: Native conformation of human von Willebrand protein. Analysis by electron microscopy and quasi-elastic light scattering. The Journal of biological chemistry 1985, 260(14):8559-8563.

3. Huizinga EG, Tsuji S, Romijn RA, Schiphorst ME, de Groot PG, Sixma J), Gros P: Structures of glycoprotein Ibalpha and its complex with von Willebrand factor A1 domain. Science (New York, NY) 2002, 297(5584):1176-1179.

4. Ruggeri ZM, Orje JN, Habermann R, Federici AB, Reininger AJ: Activation-independent platelet adhesion and aggregation under elevated shear stress. Blood 2006, 108(6):1903-1910.

5. Schneider SW, Nuschele S, Wixforth A, Gorzelanny C, Alexander-Katz A, Netz RR, Schneider MF: Shear-induced unfolding triggers adhesion of von Willebrand factor fibers. Proceedings of the National Academy of Sciences of the United States of America 2007, 104(19):7899-7903.

6. Ruggeri ZM: The role of von Willebrand factor in thrombus formation. Thromb Res 2007, 120 Suppl 1(Suppl 1):S5-9.

7. Broos K, Feys HB, De Meyer SF, Vanhoorelbeke K, Deckmyn H: Platelets at work in primary hemostasis. Blood reviews 2011, 25(4):155-167.

8. Freyssinet JM, Toti F: Formation of procoagulant microparticles and properties. Thromb Res 2010, 125 Suppl 1:S46-48.

9. Hollopeter G, Jantzen HM, Vincent D, Li G, England L, Ramakrishnan V, Yang RB, Nurden P, Nurden A, Julius D et al: Identification of the platelet ADP receptor targeted by antithrombotic drugs. Nature 2001, 409(6817):202-207.

10. Li Z, Zhang G, Le Breton GC, Gao X, Malik AB, Du X: Two waves of platelet secretion induced by thromboxane A2 receptor and a critical role for phosphoinositide 3-kinases. The Journal of biological chemistry 2003, 278(33):30725-30731.

11. Kim SO, Lim CT, Lam SC, Hall SE, Komiotis D, Venton DL, Le Breton GC: Purification of the human blood platelet thromboxane A2/prostaglandin H2 receptor protein. Biochemical pharmacology 1992, 43(2):313-322.

12. Kahn ML, Nakanishi-Matsui M, Shapiro MJ, Ishihara H, Coughlin SR: Protease-activated receptors 1 and 4 mediate activation of human platelets by thrombin. The Journal of clinical investigation 1999, 103(6):879-887.

13. Munnix IC, Cosemans JM, Auger JM, Heemskerk JW: Platelet response heterogeneity in thrombus formation. Thrombosis and haemostasis 2009, 102(6):1149-1156.

14. Jackson SP: The growing complexity of platelet aggregation. Blood 2007, 109(12):5087-5095.

15. Ruggeri ZM: Platelet adhesion under flow. Microcirculation 2009, 16(1):58-83.

16. Rana A, Westein E, Niego B, Hagemeyer CE: Shear-Dependent Platelet Aggregation: Mechanisms and Therapeutic Opportunities. Frontiers in cardiovascular medicine 2019, 6:141.

17. Dahlbäck B, Villoutreix BO: The anticoagulant protein C pathway. FEBS letters 2005, 579(15):3310-3316. 
18. Cesarman-Maus G, Hajjar KA: Molecular mechanisms of fibrinolysis. British journal of haematology 2005, 129(3):307-321.

19. Swieringa F, Spronk HMH, HeemskerkJWM, van der Meijden PEJ: Integrating platelet and coagulation activation in fibrin clot formation. Research and practice in thrombosis and haemostasis 2018, 2(3):450460.

20. Sang $Y$, Roest M, de Laat B, de Groot PG, Huskens D: Interplay between platelets and coagulation. Blood reviews 2021, 46:100733.

21. Monroe DM, Hoffman M, Roberts HR: Platelets and thrombin generation. Arteriosclerosis, thrombosis, and vascular biology 2002, 22(9):1381-1389.

22. Bevers EM, Comfurius P, Zwaal RF: Platelet procoagulant activity: physiological significance and mechanisms of exposure. Blood reviews 1991, 5(3):146-154.

23. HeemskerkJW, Mattheij NJ, Cosemans JM: Platelet-based coagulation: different populations, different functions. Journal of thrombosis and haemostasis : JTH 2013, 11(1):2-16.

24. Wood JP, Fager AM, Silveira JR, Tracy PB: Platelet-derived factor Va expressed on the surface of the activated platelet is GPI-anchored. Blood 2008, 112(11):219-220.

25. Camire RM, Kalafatis M, Simioni P, Girolami A, Tracy PB: Platelet-derived factor Va/Va Leiden cofactor activities are sustained on the surface of activated platelets despite the presence of activated protein C. Blood 1998, 91(8):2818-2829.

26. Gould WR, Silveira JR, Tracy PB: Unique in vivo modifications of coagulation factor $V$ produce a physically and functionally distinct platelet-derived cofactor: characterization of purified plateletderived factor V/Na.J Biol Chem 2004, 279(4):2383-2393.

27. Müller F, Mutch NJ, Schenk WA, Smith SA, Esterl L, Spronk HM, Schmidbauer S, Gahl WA, Morrissey $J$ H, Renné T: Platelet polyphosphates are proinflammatory and procoagulant mediators in vivo. Cell 2009, 139(6):1143-1156

28. Thomas MR, Storey RF: The role of platelets in inflammation. Thrombosis and haemostasis 2015, 114(3):449-458.

29. Plummer C, Wu H, Kerrigan SW, Meade G, Cox D, lan Douglas CW: A serine-rich glycoprotein of Streptococcus sanguis mediates adhesion to platelets via GPIb. British journal of haematology 2005, 129(1):101-109.

30. O'Seaghdha M, van Schooten CJ, Kerrigan SW, Emsley J, Silverman GJ, Cox D, Lenting PJ, Foster TJ: Staphylococcus aureus protein A binding to von Willebrand factor A1 domain is mediated by conserved IgG binding regions. The FEBS journal 2006, 273(21):4831-4841.

31. Brennan MP, Loughman A, Devocelle M, Arasu S, Chubb AJ, Foster TJ, Cox D: Elucidating the role of Staphylococcus epidermidis serine-aspartate repeat protein $\mathrm{G}$ in platelet activation. Journal of thrombosis and haemostasis : JTH 2009, 7(8):1364-1372.

32. Shannon O, Hertzén E, Norrby-Teglund A, Mörgelin M, Sjöbring U, Björck L: Severe streptococcal infection is associated with M protein-induced platelet activation and thrombus formation. Molecular microbiology 2007, 65(5):1147-1157.

33. Semple JW, Italiano JE, Jr., Freedman J: Platelets and the immune continuum. Nature reviews Immunology 2011, 11(4):264-274. 
34. Clark SR, Ma AC, Tavener SA, McDonald B, Goodarzi Z, Kelly MM, Patel KD, Chakrabarti S, McAvoy E, Sinclair GD et al: Platelet TLR4 activates neutrophil extracellular traps to ensnare bacteria in septic blood. Nature medicine 2007, 13(4):463-469.

35. Andonegui G, Kerfoot SM, McNagny K, Ebbert KV, Patel KD, Kubes P: Platelets express functional Toll-like receptor-4. Blood 2005, 106(7):2417-2423.

36. van Ommen $\mathrm{CH}$, Peters M: The bleeding child. Part I: primary hemostatic disorders. European journal of pediatrics 2012, 171(1):1-10.

37. Gresele P: Diagnosis of inherited platelet function disorders: guidance from the SSC of the ISTH. Journal of thrombosis and haemostasis : JTH 2015, 13(2):314-322.

38. Nurden AT, Nurden P: Congenital platelet disorders and understanding of platelet function. British journal of haematology 2014, 165(2):165-178.

39. Harrison P: Platelet function analysis. Blood reviews 2005, 19(2):111-123.

40. Spurgeon BEJ, Naseem KM: Platelet Flow Cytometry: Instrument Setup, Controls, and Panel Performance. Cytometry B Clin Cytom 2020, 98(1):19-27.

41. Jurk K, Shiravand Y: Platelet Phenotyping and Function Testing in Thrombocytopenia.J Clin Med 2021, 10(5)

42. Cattaneo M, Cerletti C, Harrison P, Hayward CP, Kenny D, Nugent D, Nurden P, Rao AK, Schmaier AH, Watson SP et al: Recommendations for the Standardization of Light Transmission Aggregometry: A Consensus of the Working Party from the Platelet Physiology Subcommittee of SSC/ISTH. Journal of thrombosis and haemostasis : JTH 2013.

43. Hayward CP, Pai M, Liu Y, Moffat KA, Seecharan J, Webert KE, Cook RJ, Heddle NM: Diagnostic utility of light transmission platelet aggregometry: results from a prospective study of individuals referred for bleeding disorder assessments. Journal of thrombosis and haemostasis : JTH 2009, 7(4):676-684.

44. Gresele P, Harrison P, Bury L, Falcinelli E, Gachet C, Hayward CP, Kenny D, Mezzano D, Mumford AD, Nugent D et al: Diagnosis of suspected inherited platelet function disorders: results of a worldwide survey. Journal of thrombosis and haemostasis : JTH 2014, 12(9):1562-1569.

45. Shattil SJ, Cunningham M, Hoxie JA: Detection of activated platelets in whole blood using activationdependent monoclonal antibodies and flow cytometry. Blood 1987, 70(1):307-315.

46. Lindahl TL, Fagerberg $\mathrm{H}$, Larsson A: A new flow cytometric method for measurement of von Willebrand factor activity. Scandinavian journal of clinical and laboratory investigation 2003, 63(3):217-223.

47. Giannini S, Cecchetti L, Mezzasoma AM, Gresele P: Diagnosis of platelet-type von Willebrand disease by flow cytometry. Haematologica 2010, 95(6):1021-1024.

48. Boknas N, Macwan AS, Sodergren AL, Ramstrom S: Platelet function testing at low platelet counts: When can you trust your analysis? Research and practice in thrombosis and haemostasis 2019, 3(2):285-290.

49. Michelson AD, Barnard Mr Fau - Hechtman HB, Hechtman Hb Fau - MacGregor H, MacGregor H Fau - Connolly RJ, Connolly Rj Fau - Loscalzo J, Loscalzo J Fau - Valeri CR, Valeri CR: In vivo tracking of platelets: circulating degranulated platelets rapidly lose surface P-selectin but continue to circulate and function. (0027-8424 (Print)).

50. Evangelista V, Smyth SS: Chapter 16 - Interactions Between Platelets, Leukocytes and the Endothelium 
A2 - Michelson, Alan D. In: Platelets (Third Edition). edn.: Academic Press; 2013: 295-312.

51. Michelson AD, Barnard MR, Krueger LA, Valeri CR, Furman MI: Circulating monocyte-platelet aggregates are a more sensitive marker of in vivo platelet activation than platelet surface P-selectin: studies in baboons, human coronary intervention, and human acute myocardial infarction. Circulation 2001, 104(13):1533-1537.

52. Furman MI, Benoit SE, Barnard MR, Valeri CR, Borbone ML, Becker RC, Hechtman HB, Michelson AD: Increased platelet reactivity and circulating monocyte-platelet aggregates in patients with stable coronary artery disease. Journal of the American College of Cardiology 1998, 31(2):352-358.

53. Totani L, Evangelista V: Platelet-leukocyte interactions in cardiovascular disease and beyond. Arteriosclerosis, thrombosis, and vascular biology 2010, 30(12):2357-2361.

54. Kopp CW, Gremmel T, Steiner S, Seidinger D, Minar E, Maurer G, Huber K: Platelet-monocyte cross talk and tissue factor expression in stable angina vs. unstable angina/non ST-elevation myocardial infarction. Platelets 2011, 22(7):530-536.

55. Furman MI, Barnard MR, Krueger LA, Fox ML, Shilale EA, Lessard DM, Marchese P, Frelinger AL, 3rd, Goldberg RJ, Michelson AD: Circulating monocyte-platelet aggregates are an early marker of acute myocardial infarction. Journal of the American College of Cardiology 2001, 38(4):1002-1006.

56. Rutten B, Tersteeg C, Vrijenhoek JE, van Holten TC, Elsenberg EH, Mak-Nienhuis EM, de Borst GJ, Jukema JW, Pijls NH, Waltenberger J et al: Increased platelet reactivity is associated with circulating platelet-monocyte complexes and macrophages in human atherosclerotic plaques. PloS one 2014, 9(8):e105019.

57. Lippi G, Favaloro EJ: Hemostasis practice: state-of-the-art. Journal of Laboratory and Precision Medicine 2018, 3:67-67.

58. Nogami K: The utility of thromboelastography in inherited and acquired bleeding disorders. British journal of haematology 2016, 174(4):503-514.

59. Tripodi A: Thrombin Generation Assay and Its Application in the Clinical Laboratory. Clinical chemistry 2016, 62(5):699-707.

60. Hemker HC, Giesen P, Al Dieri R, Regnault V, de Smedt E, Wagenvoord R, Lecompte T, Beguin S: Calibrated automated thrombin generation measurement in clotting plasma. Pathophysiology of haemostasis and thrombosis 2003, 33(1):4-15.

61. Ten Cate H: Thrombin generation in clinical conditions. Thromb Res 2012, 129(3):367-370.

62. Wan J, Konings J, Yan Q, Kelchtermans H, Kremers R, de Laat B, Roest M: A novel assay for studying the involvement of blood cells in whole blood thrombin generation. Journal of thrombosis and haemostasis : JTH 2020, 18(6):1291-1301.

63. Achison M, Joel C, Hargreaves PG, Sage SO, Barnes MJ, Farndale RW: Signals elicited from human platelets by synthetic, triple helical, collagen-like peptides. Blood coagulation \& fibrinolysis : an international journal in haemostasis and thrombosis 1996, 7(2):149-152.

64. Polgár J, Clemetson JM, Kehrel BE, Wiedemann M, Magnenat EM, Wells TN, Clemetson KJ: Platelet activation and signal transduction by convulxin, a C-type lectin from Crotalus durissus terrificus (tropical rattlesnake) venom via the p62/GPVI collagen receptor. The Journal of biological chemistry 1997, 272(21):13576-13583. 



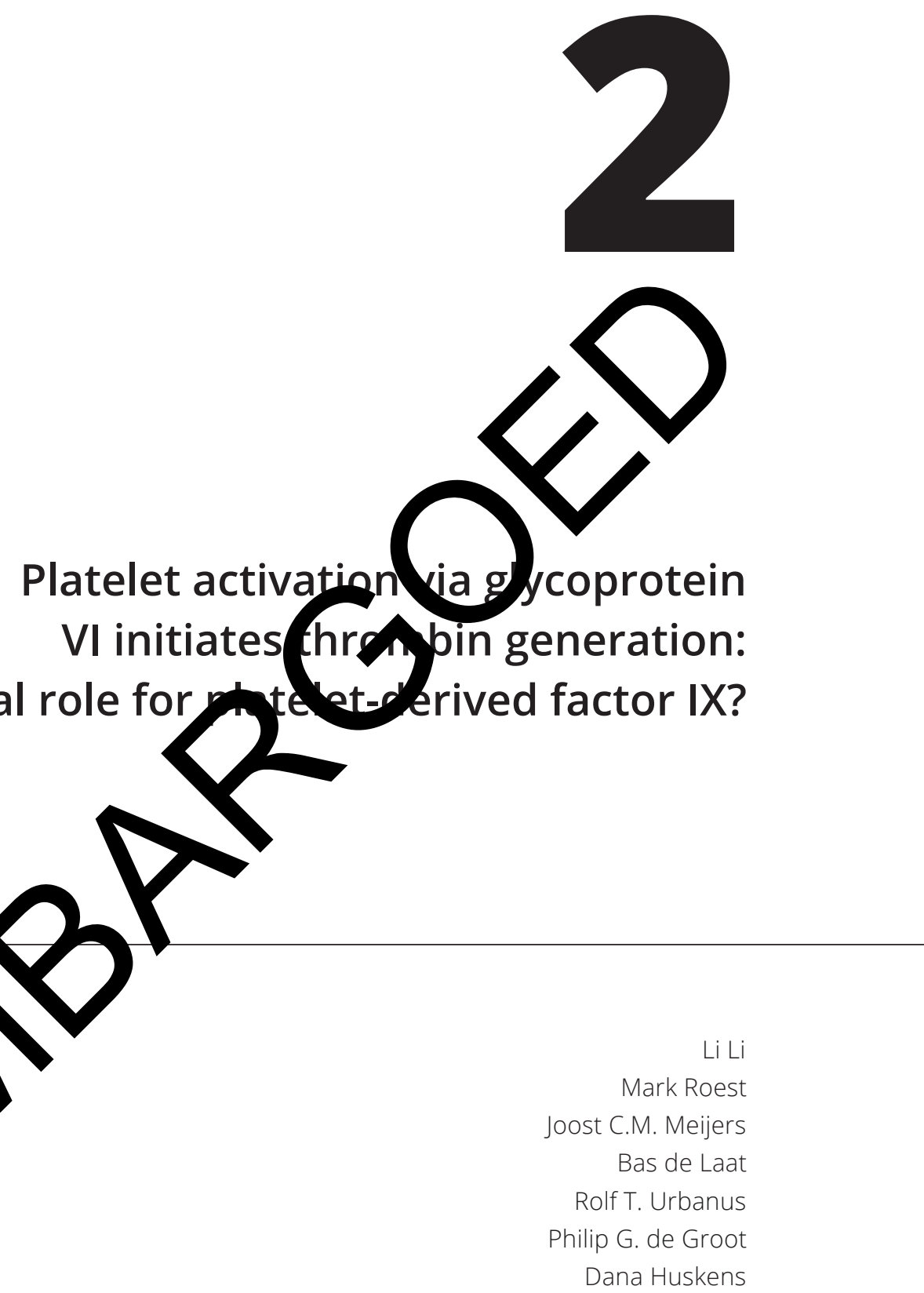




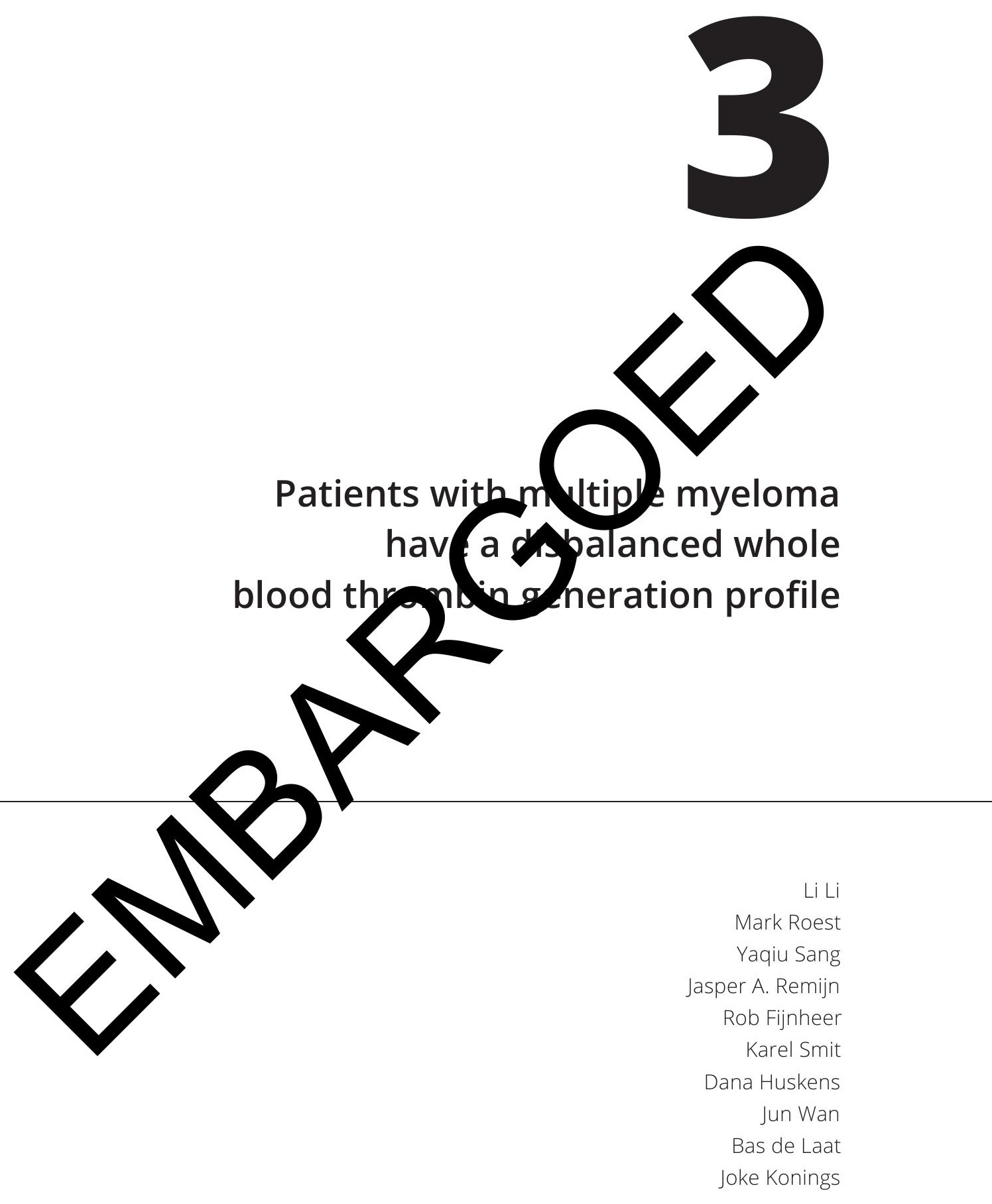




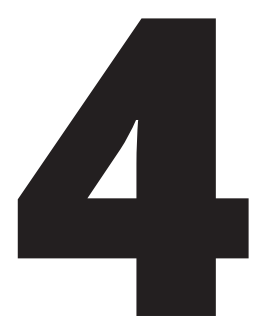

\section{Acute exacerbations of COPD are associated with a prothrombotic state through platelet- monocyte complexes, endothelial activation and increased thrombin generation}

Dana Huskens

Janine J.J. Hulstein

Mark Roest

Philip G. de Groot

Hugo ten Cate

Bas de Laat

Jasper A. Remijn

Sami O. Simons 


\section{ABSTRACT}

\section{Introduction}

Patients with chronic obstructive pulmonary disease (COPD) are at increased risk for cardiovascular events, particularly following an acute exacerbation (AE-COPD). Exacerbations are associated with increased systemic inflammation, which may drive coagulation. This prospective cohort study aimed to determine how an AE-COPD affects platelet activation, the endothelium, plasmatic coagulation and fibrinolysis, and its association with systemic inflammation.

\section{Materials and methods}

Fifty-two patients with an AE-COPD were included. Blood samples at admission, at day 3 of treatment and at convalescence were available for 32 patients. Platelet-monocyte complex (PMC) formation, monocyte Mac-1 expression and platelet (re)activity (P-selectin expression, allbß3 activation) were measured by flow cytometry. Von Willebrand Factor (VWF), thrombin generation (TG) and clot lysis time (CLT) were determined as measures of endothelial activation, plasmatic coagulation and fibrinolysis, respectively.

\section{Results}

Exacerbations were associated with increased PMCs (MFI 31.3 vs 23.8, $p=0.004$ ) and Mac-1 (MFI 38.2 vs 34.8, p = 0.006) compared to convalescence, but not with changes in platelet (re)activity. VWF (antigen, activity, active fraction) and TG (peak, ETP and velocity index) were all significantly higher during AE-COPD compared to convalescence. PMCs, Mac-1, VWF and TG were positively associated with systemic inflammation (CRP). CLT was prolonged in AE-COPD patients with systemic inflammation. Moreover, platelet hyperreactivity on admission was associated with an increased risk for exacerbation relapse.

\section{Conclusions}

Acute exacerbations are associated with an inflammation-associated prothrombotic state, characterized by increased PMCs, endothelial activation and plasmatic coagulation. Our findings provide direction for future studies on biomarkers predicting the risk of exacerbation relapse and cardiovascular events. 


\section{INTRODUCTION}

Cardiovascular disease (CVD) is the most important comorbidity associated with chronic obstructive pulmonary disease (COPD) due to its impact on overall prognosis, including mortality [1]. Importantly, the prevalence of thrombotic events, such as myocardial infarction, stroke and pulmonary embolism, is known to increase during and shortly after acute exacerbations of COPD (AE-COPD), but the precise mechanisms remain unclear [2-4].

COPD exacerbations are most frequently caused by viral and/or bacterial infections [5]. The inflammatory response that is needed to control the infection, might also affect coagulation during an AE-COPD [6]. First, inflammatory stimuli may induce platelet activation, resulting in allb $\beta 3$ (glycoprotein [GP]Ilbllla) activation, thereby mediating platelet adhesion and aggregation [7,8]. Activated platelets also secrete procoagulant substances, such as ADP and fibrinogen, as well as P-selectin [7]. P-selectin can bind to its counterreceptor P-selectin glycoprotein ligand-1 (PSGL-1), constitutively expressed on the membrane of monocytes. Binding of platelets to monocytes results in the expression of the monocyte integrin Mac-1 [9]. Several studies have demonstrated the importance of these platelet-monocyte complexes (PMCS) as a marker of platelet activation $[8,10]$, but also as an early process in the pathogenesis of atherothrombosis [11-13]. Second, inflamed endothelium releases von Willebrand Factor (VWF), which mediates adhesion of platelets to the vessel wall and subsequent thrombus growth [14]. Third, inflammation also promotes thrombin generation (TG). Thrombin is the key effector of plasmatic coagulation, but also has an array of effects on endothelial cells, monocytes, and platelets [15].

The interplay between inflammation and coagulation during AE-COPD suggests that activation of platelets, the endothelium and the plasmatic coagulation may all play a role in the pathogenesis of CVD in these patients. Few studies investigated platelet activation during AE-COPD, and found increased PMCs without changes in platelet P-selectin expression [16, 17]. In addition, increased VWF antigen (VWF:Ag) levels and VWF ristocetin cofactor activity (VWF:RCo) have been reported in AE-COPD patients [18]. Active VWF and VWF propeptide (VWFpp) provide additional information about VWF processing and the chronicity of endothelial activation, but these have not been studied in AE-COPD $[19,20]$. Moreover, whereas a previous study found increased thrombinantithrombin complex (TAT), a surrogate marker of thrombin formation during AECOPD, full thrombin generation profiles have not been determined in AE-COPD [21]. Lastly, previous reports mostly focused on the effects of an AE-COPD on coagulation, while limited information is available on how fibrinolysis is affected. 
Together, platelets, endothelium and circulating coagulation and fibrinolytic factors determine the overall thrombotic tendency, but they have been scarcely studied together in AE-COPD patients. Therefore, the current study aimed to determine how an AE-COPD affects platelet activity and PMC formation, endothelial function, plasmatic coagulation and fibrinolysis. We hypothesized that during an AE-COPD all pro-coagulant factors are in a heightened state of activation, while fibrinolysis may be decreased, and that these changes are associated with increased systemic inflammation.

\section{METHODS}

\section{Study subjects and design}

In this prospective observational cohort study, we recruited 52 subjects with a confirmed diagnosis of an AE-COPD (in accordance with the GOLD 2019 definition [22]), admitted to the Department of Respiratory Medicine of the Gelre Hospitals Apeldoorn, the Netherlands, between December 2018 and June 2019. Inclusion criteria were: (1) age $\geq 40$ years, (2) current or former smoker ( $\geq 10$ pack-years) (3) moderate to severe COPD (GOLD $\geq 2$ ) and (4) hospital admission due to an AE-COPD. An AE-COPD was defined as an acute worsening of respiratory symptoms requiring additional therapy [22]. Exclusion criteria were: use of platelet function inhibitors (therapy with acetylsalicylic acid was allowed), vitamin $\mathrm{K}$ antagonists or direct oral anticoagulants; concomitant diagnosis of asthma, hepatic and/or renal failure, malignancies (excluding basal cell carcinoma) and chronic inflammatory diseases (including systemic lupus erythematosus, chronic kidney disease, rheumatoid arthritis and all other inflammatory joint diseases, psoriasis and inflammatory bowel diseases). Eligible patients were asked to participate within $24 \mathrm{~h}$ after admission. All patients received exacerbation treatment in accordance with national Dutch guidelines and were treated with steroids (100\%) and antibiotics (27\%). The majority of patients (87\%) also received prophylactic low molecular weight heparin on admission based on current guidelines on antithrombotic therapy.

\section{Ethics}

Patients were included after written informed consent, complying with the Declaration of Helsinki (version 2013). This study was approved by the Medical Research Ethics Committee of Maastricht University, the Netherlands (METC azM/UM, reference NL66067.068.18).

\section{Blood collection and processing}

Blood samples were obtained during AE-COPD (within $24 \mathrm{~h}$ of admission, "AE-COPD"), 3 days later ("day 3 of treatment") and (when clinically stable) on average 8 weeks (min 6, max 12 weeks) after discharge ("convalescence"). Of note, the timepoint at day 3 of their hospital stay was included as patients received exacerbation treatment that could 
potentially affect coagulation. Venous blood was collected by venipuncture into EDTA and 3.2\% sodium citrate vacuum tubes (BD Vacutainer System, Franklin Lakes, USA). Blood cell counts, routine coagulation tests (additional detail on these tests and VWF assays is provided in an online data supplement) and flow cytometric assays were performed within $4 \mathrm{~h}$ after blood collection. The remaining blood was centrifuged twice at $2840 \mathrm{~g}$ for $10 \mathrm{~min}$. Platelet-poor plasma (PPP) was stored at $-80{ }^{\circ} \mathrm{C}$ until further analyses.

\section{PMCs and Mac-1 expression}

Whole blood $(20 \mu \mathrm{L})$ was incubated for 15 min with a $20 \mu \mathrm{L}$ reaction mixture consisting of monoclonal antibodies against monocyte CD14 (APC-conjugated) (Becton Dickinson) and platelet allbß3 (anti-CD41a PE-conjugated) for PMCs or antibodies against monocyte CD14 (APC-conjugated) and CD11b (FITC-conjugated) for Mac-1 expression. Subsequently, BD FACS ${ }^{\text {TM }}$ Lysing solution (Becton-Dickinson, diluted 10-fold with milliQ) was added, vortexed and incubated for $10 \mathrm{~min}$ at room temperature. Samples were stored at $4{ }^{\circ} \mathrm{C}$ until analysis (on the same day) by flow cytometry (Navios, Beckman Coulter, Woerden, the Netherlands). Gating was performed as described previously [16].

\section{Platelet activation and reactivity}

Flow cytometric analysis of the activation of allbß3 and expression of P-selectin (CD62P) was used to determine platelet activation in response to thrombin-activated peptide (TRAP), cross-linked collagen-related peptide (CRP-XL) and adenosine diphosphate (ADP), as described previously [23]. Results are expressed as median fluorescent intensity (MFI), calculated by subtracting the MFI of the unstimulated control from the $\mathrm{MFI}$ of the condition with agonist.

\section{Plasmatic coagulation}

Calibrated automated thrombinography (CAT) TG in PPP was performed as described previously [24].

\section{Fibrinolysis}

Fibrinolysis was assessed by measuring the clot lysis time (CLT), as described previously [25]. Samples were tested in triplicate and preheated at $37^{\circ} \mathrm{C}$ for $10 \mathrm{~min}$. A total of $80 \mu \mathrm{l}$ plasma was incubated $\left(10 \mathrm{~min}, 37^{\circ} \mathrm{C}\right)$ with $20 \mu \mathrm{l}$ of a bovine serum albumin (5\%) solution containing tissue plasminogen activator (t-PA, Actilyse, Boehringer Ingelheim, Germany), TF and PL at final concentrations of $200 \mathrm{ng} / \mathrm{ml}, 5 \mathrm{pM}$ and $4 \mu \mathrm{M}$, respectively. Fibrin clot formation was started by adding $20 \mu \mathrm{l}$ of a preheated $\mathrm{CaCl}_{2} / \mathrm{BSA} 60$ solution (final concentration $16.7 \mathrm{mM}$ ). The optical density at $405 \mathrm{~nm}$ was measured every $20 \mathrm{~s}$ for $1 \mathrm{~h}$ at $37^{\circ} \mathrm{C}$ using a SpectraMax M2 plate reader (Molecular Devices, Sunnyvale, CA, USA). The CLT was calculated as the time from half-maximal fibrin formation to halfmaximal fibrin degradation. 


\section{Statistical analysis}

Conformity to the normal distribution was evaluated with Q-Q plots and the Shapiro-Wilk test. Categorical variables were expressed as percentages and continuous variables as mean \pm SD or median (interquartile range, IQR). Comparisons between the three timepoints (for 32 patients with complete follow-up) were performed by repeated-measures ANOVA with post-hoc Bonferroni test or non-parametric Friedman tests with posthoc Wilcoxon signed-rank tests and Bonferroni correction for multiple comparisons. Comparisons between two independent groups (at baseline, all 52 subjects) were performed by independent samples t-test or non-parametric Mann-Whitney $U$ test. Associations were assessed using Spearman's rank correlation tests (all 52 subjects). A p-value of 0.05 was considered statistically significant. All analyses were performed using Statistical Package for Social Sciences (SPSS Inc., Chicago, IL, USA) version 25.

\section{RESULTS}

\section{Baseline characteristics of the study population}

Fifty-two patients admitted to the Gelre Hospitals Apeldoorn with a confirmed AE-COPD were included in this study (Fig. 1). Three patients (6\%) died during the follow-up as a consequence of respiratory failure. Moreover, 17 patients did not show up for the blood draw at convalescence (on average 8 weeks after discharge). Thus, blood samples at all 3 time points were obtained for 32 subjects (Fig. 1, Table S1).

\section{Eligible based on inclusion criteria $(n=171)$}

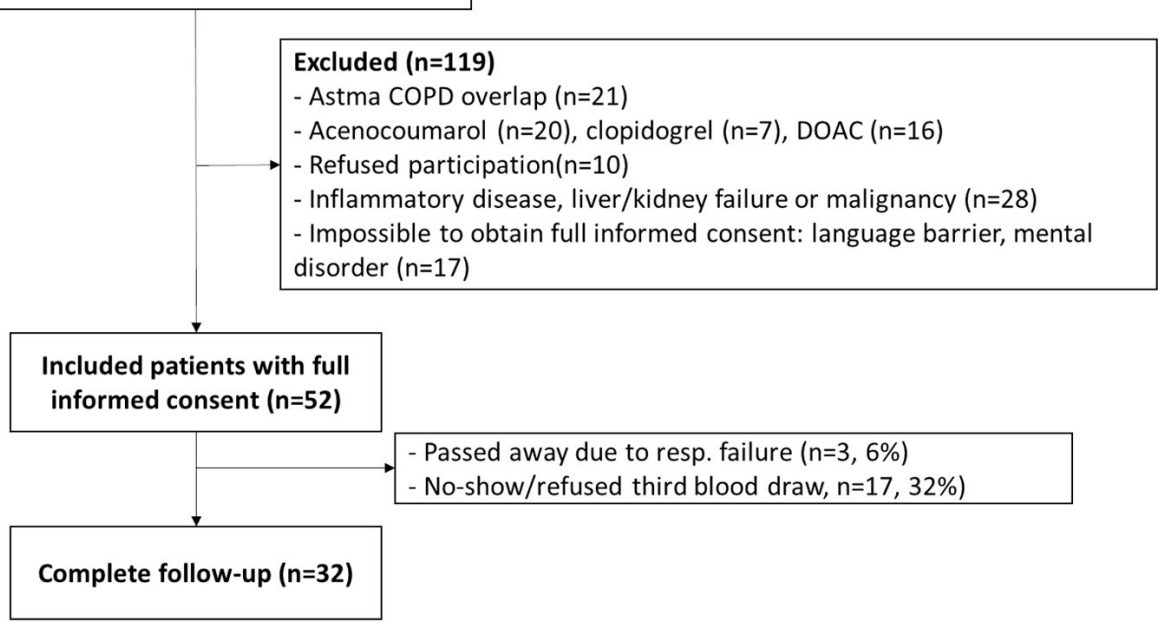

Figure 1. Inclusion flow-chart. 
Baseline characteristics of the study participants are summarized in Table 1. In approximately half of the patients, the AE-COPD had a proven infectious cause. At presentation, 26 of the patients had CRP levels $>10 \mathrm{mg} / \mathrm{L}$, indicative of systemic inflammation, of which 18 had a proven viral $(n=6)$, bacterial $(n=9)$ or combined $(n=3)$ infectious cause. Fifteen patients (28.8\%) had a history of CVD, for which they all received aspirin. Data are presented as the mean \pm SD or median (IQR) for normally distributed and non-parametric continuous variables, respectively, and as the absolute number [percentage of total ( $n=52)$ ] for categorical variables. * Hypoxemia was defined as oxygen saturation $<90 \%$ while breathing room air. † Including deep venous thrombosis, ischemic stroke, transient ischemic attack, pulmonary embolism, acute coronary syndrome, heart failure. Abbreviations: FEV1, forced expiratory volume in $1 \mathrm{~s}$; VC, vital capacity; LMWH: prophylactic low-molecular-weight heparin; SAMA/LAMA, short-acting/long-acting muscarinic antagonist; SABA/LABA, shortacting/long-acting beta-agonists; ICS, inhaled corticosteroids; CRP, C-reactive protein; AECOPD, acute exacerbation of COPD; CVD, cardiovascular disease.

Table 1. Baseline patient characteristics.

\begin{tabular}{|c|c|}
\hline Variable & Mean \pm SD; Median (IQR); $\mathrm{n}[\%]$ \\
\hline $\mathrm{N}$ & 52 \\
\hline Age, years & $66.5 \pm 10.4$ \\
\hline Male, $n[\%]$ & $21[40.4 \%]$ \\
\hline Body mass index, $\mathrm{kg} / \mathrm{m}^{2}$ & $25.1(5.3)$ \\
\hline Current smoker, $n[\%]$ & $16[30.8 \%]$ \\
\hline Smoking history, pack-years & $31.5 \pm 12.9$ \\
\hline \multicolumn{2}{|l|}{ GOLD score, $n[\%]$} \\
\hline II & 15 [28.8\%] \\
\hline III & 22 [42.3\%] \\
\hline IV & 15 [28.8\%] \\
\hline \multicolumn{2}{|l|}{ Pulmonary function } \\
\hline FEV1, I & $0.92(0.53)$ \\
\hline FEV1 predicted, $\%$ & $36.0(17.4)$ \\
\hline VC, I & $2.5 \pm 0.9$ \\
\hline VC predicted, \% & $74.6 \pm 18.7$ \\
\hline Tiffeneau index, $\%$ & $37.0(16.0)$ \\
\hline \multicolumn{2}{|l|}{ Inhalation medication, $n[\%]$} \\
\hline Short-acting bronchodilators (SAMA/SABA) & $45[86.5 \%]$ \\
\hline Monotherapy long-acting (LABA/LAMA) & $7[13.5 \%]$ \\
\hline Double therapy (LABA + LAMA/LABA + ICS) & 16 [30.8\%] \\
\hline Triple therapy (LABA + LAMA + ICS) & $25[48.1 \%]$ \\
\hline Emphysema, $n[\%]$ & $34[65.4 \%]$ \\
\hline Exacerbation rate, $n$ per year & $2(2)$ \\
\hline Hypoxemia* at presentation, $n[\%]$ & 19 [36.5\%] \\
\hline CRP $>10 \mathrm{mg} / \mathrm{L}$ at presentation, $n[\%]$ & $26[50.0 \%]$ \\
\hline Infectious cause of AE-COPD, $n[\%]$ & $27[51.9 \%]$ \\
\hline Viral infection & 12 [23.1\%] \\
\hline Bacterial infection & $10[19,2 \%]$ \\
\hline Combined bacterial and viral infection & $5[9.6 \%]$ \\
\hline Duration of hospitalization, days & $6(4)$ \\
\hline LMWH during hospital stay, $n[\%]$ & $45[86.5 \%]$ \\
\hline History of CVD ${ }^{\dagger}, n[\%]$ & $15[28.8 \%]$ \\
\hline On aspirin therapy, $n[\%]$ & $15[28.8 \%]$ \\
\hline
\end{tabular}

Data are presented as the mean \pm SD or median (IQR) for normally distributed and non-parametric continuous variables, respectively, and as the absolute number [percentage of total $(n=52)$ ] for categorical variables. * Hypoxemia was defined as oxygen saturation $<90 \%$ while breathing room 
air. † Including deep venous thrombosis, ischemic stroke, transient ischemic attack, pulmonary embolism, acute coronary syndrome, heart failure. Abbreviations: FEV1, forced expiratory volume in $1 \mathrm{~s}$; VC, vital capacity; LMWH: prophylactic low-molecular-weight heparin; SAMA/LAMA, shortacting/long-acting muscarinic antagonist; SABA/LABA, short-acting/long-acting beta-agonists; ICS, inhaled corticosteroids; CRP, C-reactive protein; AE-COPD, acute exacerbation of COPD; CVD, cardiovascular disease.

\section{Increased PMC formation and monocyte activation during AE-COPD}

PMCs were significantly increased during AE-COPD (median 31.3, IQR 15.3) compared to after three days of treatment (median 20.8, IQR 8.2, p < 0.001) and convalescence (median 23.8, IQR 7.9, $\mathrm{p}=0.004$ ) (Fig. 2A). There was a small, positive correlation between PMCs and CRP levels (Fig. 2B, open circles).

Monocyte Mac-1 expression was also increased during AE-COPD (median 38.2, IQR 20.0) compared to during treatment (median 34.0, IQR 9.9, $p=0.001$ ) and convalescence (median 34.8, IQR 12.5, $p=0.006$ ) (Fig. 2C). There was a positive correlation between monocyte Mac-1 expression and PMC formation ( $r=0.353, p<0.001$ ) (Fig. 2D). Patients with CRP levels $>10 \mathrm{mg} / \mathrm{L}$ at baseline had significantly higher monocyte Mac-1 expression (median MFI 38.7, IQR 14.5) than patients with CRP below this cut-off (33.5, IQR 11.4) $(p=0.002)$ and the correlation between CRP and Mac- 1 was also significant $(r=0.423$, $p<0.001$ ) (Fig. 2B, closed circles).

\section{Platelet P-selectin expression and allb $\beta 3$ activation are not affected by AE-COPD}

P-selectin expression, either unstimulated or in response to TRAP, CRP-XL and ADP, was not significantly different between AE-COPD, day 3 of treatment and convalescence (Table 2, top half). Similarly, activation of the P-selectin expression and allb $\beta 3$ activation were measured by flow cytometry as markers of platelet activation. Whole blood was incubated without (unstimulated, representing spontaneous/"in vivo" platelet activation) or with one of the platelet agonists TRAP, CRP or ADP. Data are presented as the median $(\mathrm{IQR})(\mathrm{n}=32)$. Abbreviations: TRAP, thrombin receptor activating peptide; CRP-XL, crosslinked collagen-related peptide; ADP, adenosine diphosphate; MFI, median fluorescent intensity; ns, non-significant. 

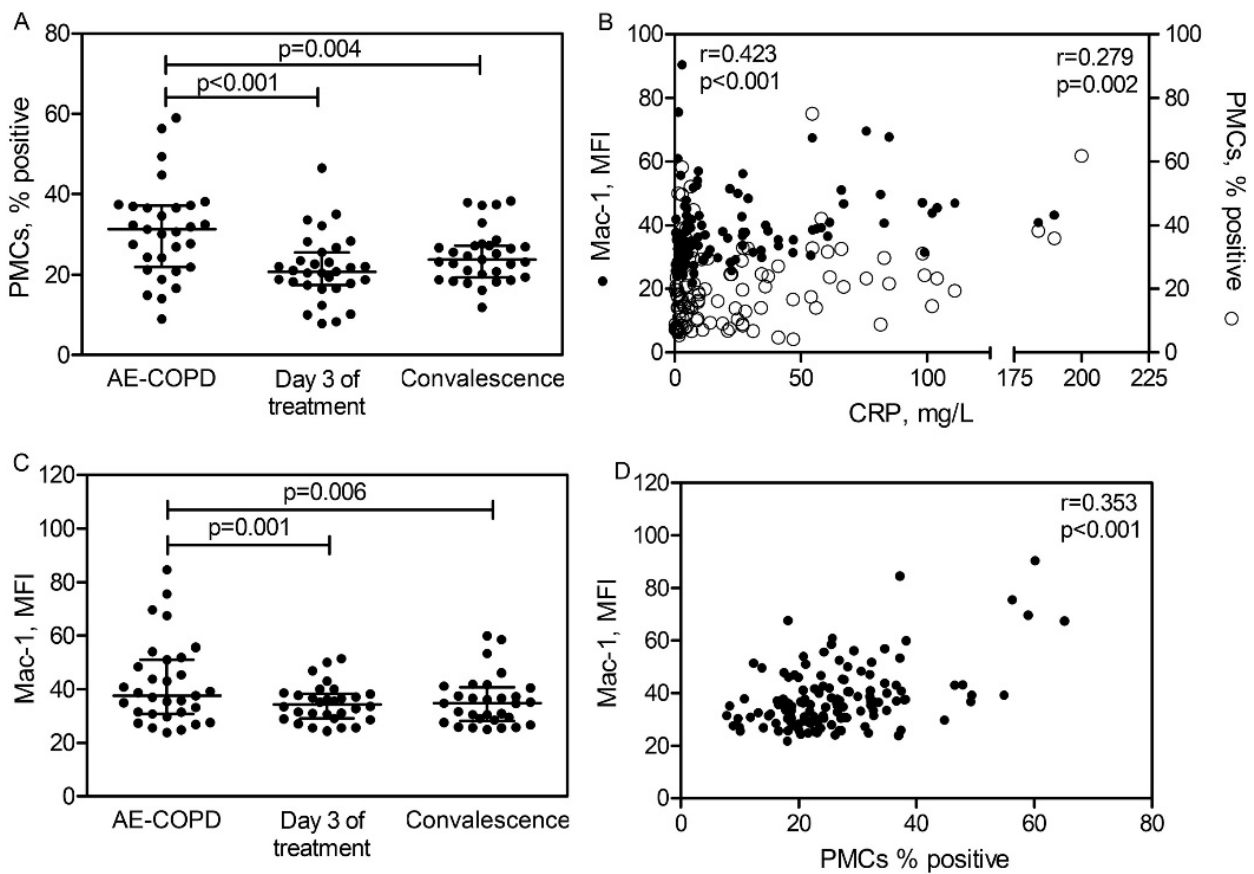

Figure 2. Platelet-monocyte complexes and monocyte Mac-1 expression during and after AE-COPD. Circulating PMCS (A) and Mac-1 expression (C) were measured at the 3 time points $(n=32)$. The horizontal line represents the median, whiskers indicate the first and third quartiles. Significant differences are indicated with corresponding p-values. (B) The correlation between PMCs (right axis, open circles with dashed linear regression) and Mac-1 expression (left axis, closed circles with solid linear regression) with inflammation, measured as CRP. Corresponding Spearman correlation coefficients and $\mathrm{p}$-values are indicated in the upper left and right corners for Mac-1 and PMCs, respectively (data from all three time points, $n=52$ ). (D) Correlation between PMCs and Mac-1 expression (data from all 3 time points, $n=52$ ). Abbreviations: PMCs, platelet-monocyte complexes; MFI, median fluorescent intensity.

Table 2. Platelet (re)activity during and after AE-COPD.

\begin{tabular}{|c|c|c|c|c|}
\hline Parameter & AE-COPD & Day 3 of treatment & Convalescence & P-value \\
\hline \multicolumn{5}{|c|}{ P-selectin expression (MFI) } \\
\hline Unstimulated & $0.32(0.07)$ & $0.32(0.08)$ & $0.32(0.12)$ & $n s, p=0.552$ \\
\hline TRAP & $26.2(8.6)$ & $25.0(10.1)$ & $23.9(6.0)$ & $n s, p=0.156$ \\
\hline CRP-XL & $24.4(9.4)$ & $24.3(7.8)$ & $24.8(8.0)$ & $n s, p=0.055$ \\
\hline ADP & $5.8(8.0)$ & $5.6(5.1)$ & $6.4(7.2)$ & $n s, p=0.129$ \\
\hline \multicolumn{5}{|c|}{ allb $\beta 3$ activation (MFI) } \\
\hline Unstimulated & $0.53(0.30)$ & $0.57(0.27)$ & $0.64(0.27)$ & $n s, p=0.249$ \\
\hline TRAP & $2.6(2.2)$ & $2.1(2.6)$ & $3.2(2.7)$ & $n s, p=0.051$ \\
\hline CRP-XL & $19.1(10.5)$ & $20.9(13.1)$ & $20.2(9.2)$ & $n s, p=0.897$ \\
\hline ADP & $13.3(7.6)$ & $11.9(9.2)$ & $13.6(11.0)$ & $n s, p=0.084$ \\
\hline
\end{tabular}




\section{Endothelial activation during AE-COPD}

Endothelial function was evaluated by measuring various VWF parameters. VWF:Ag levels were significantly elevated during AE-COPD (median 218\%, IQR 113\%) and at day 3 of treatment (median 217\%, IQR 126\%) compared to convalescence (median 182\%, IQR 110\%) (Fig. 3A). Similarly, VWF:RCo activity was increased during AE-COPD (median 157\%, IQR 89\%, $p=0.009$ ) and day 3 of treatment (median 158\%, IQR 64\%, $p=0.002$ ) compared to convalescence (median 135\%, IQR 71\%) (Fig. 3B).
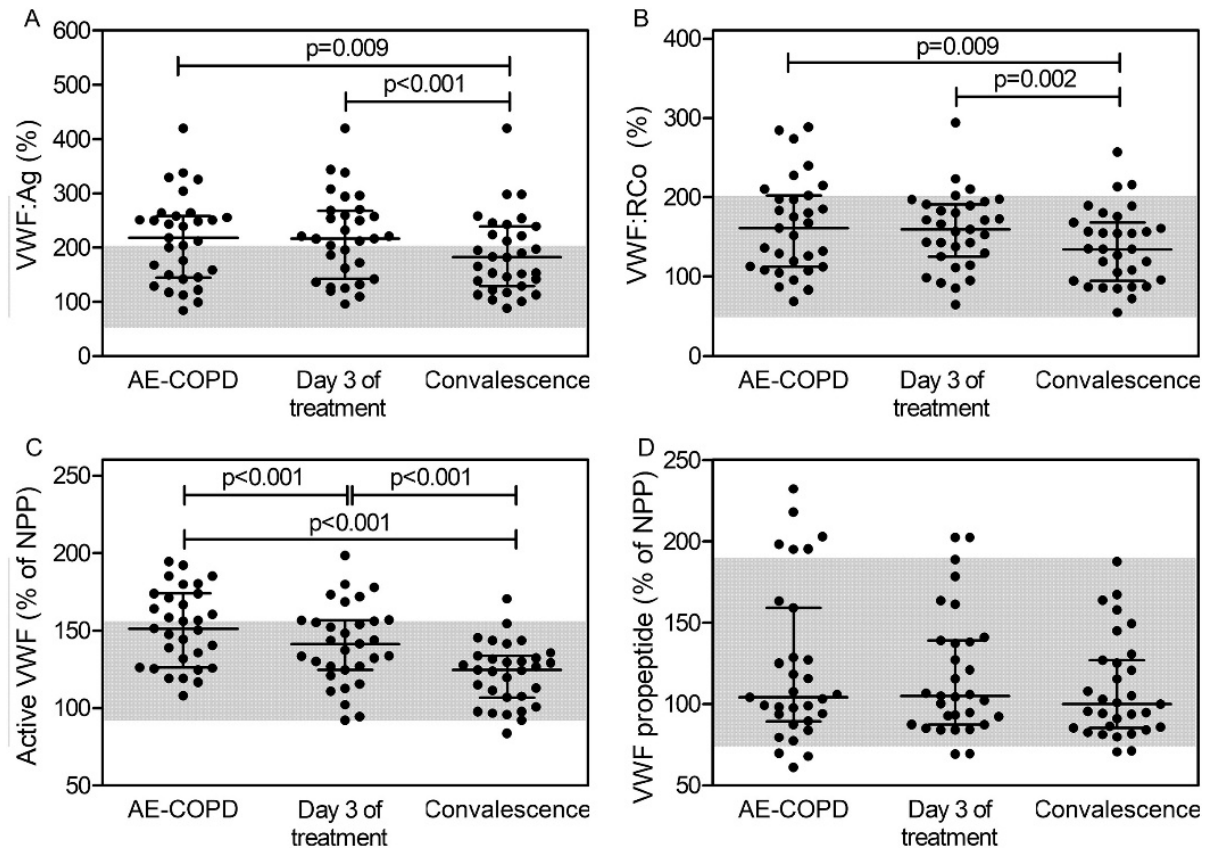

Figure 3. Endothelial cell activation measured by circulating VWF parameters. (A) VWF:Ag, (B) VWF:RCo, (C) active VWF and (D) VWF propeptide at the 3 sample time points. The horizontal line represents the median, whiskers indicate the first and third quartiles. Significant differences are indicated with corresponding p-values $(n=32)$. Abbreviations: NPP, normal pooled plasma; VWF:Ag, VWF antigen; VWF:RCo, VWF ristocetin cofactor activity; AE-COPD, acute exacerbation of COPD.

Active VWF levels were highest during AE-COPD (median 151\%, IQR 48), followed by a drop during treatment (median 142\%, IQR 32, p < 0.001) and lowest levels at convalescence (median 125\%, IQR 27, p < 0.001) (Fig. 3C). In contrast, VWFpp levels remained stable over time (Fig. 3D). CRP correlated significantly with active VWF (Spearman $r=0.395$, $p<0.001$ ), VWF:Ag (Spearman $r=0.475, p<0.001$ ) and VWFpp (Spearman $r=0.312$, $p=0.001$ ) levels. 


\section{Plasmatic coagulation is increased during AE-COPD}

The total (ETP) and maximum (peak) amount of thrombin generated and the velocity of thrombin formation (VI) were all significantly increased during AE-COPD compared to convalescence, at all concentrations (0,1 and 5 pM) of TF (Table 3). The ETP (at 1 and 5 pM TF) was also increased during AE-COPD (1.502 \pm 356 and $1.503 \pm 335 \mathrm{nM}$ min, respectively) compared to day 3 of treatment (1.440 \pm 332 and $1.418 \pm 271 \mathrm{nM}$ min, respectively, both $p<0.001)$. The "time-parameters" lagtime and TTP were not significantly different between the timepoints.

Table 3. Thrombin generation during and after AE-COPD.

\begin{tabular}{|c|c|c|c|c|}
\hline & AE-COPD & Day 3 treatment & Convalescence & p-value \\
\hline \multicolumn{5}{|l|}{$5 \mathrm{pM}$ TF } \\
\hline LT, S & $3.6(3.3-3.9)$ & $3.3(3.0-4.0)$ & $3.6(3.1-4.0)$ & $n s, p=0.103$ \\
\hline ETP, nM.min & $1503 \pm 335^{* \dagger}$ & $1418 \pm 271 \dagger$ & $1400 \pm 360 *$ & $\begin{array}{l}* p=0.015 \\
t p<0.001\end{array}$ \\
\hline Peak, $n M$ & $283(240-321)^{\star}$ & $260(230-308)$ & $251(209-290)^{*}$ & ${ }^{*} p=0.003$ \\
\hline TTP, s & $6.6(6.0-7.0)$ & $6.3(6.0-7.3)$ & $6.7(6.2-7.3)$ & $n s, p=0.350$ \\
\hline $\mathrm{Vl}, n M / \min$ & $101.3 \pm 39.0 *$ & $91.8 \pm 39.8$ & $80.2 \pm 36.1^{*}$ & ${ }^{*} p=0.01$ \\
\hline \multicolumn{5}{|l|}{$1 \mathrm{pM} \mathrm{TF}$} \\
\hline LT, S & $5.8(5.4-6.6)$ & $6.0(5.3-6.8)$ & $6.0(5.2-6.6)$ & $n s, p=0.095$ \\
\hline ETP, nM.min & $1502 \pm 356 * \dagger$ & $1440 \pm 332 \dagger$ & $1375 \pm 361$ * & $\begin{array}{l}* p=0.008 \\
t p<0.001\end{array}$ \\
\hline Peak, $n M$ & $303(261-350)^{\star}$ & $278(233-328)$ & $265(218-291)^{\star}$ & ${ }^{*} p=0.001$ \\
\hline TTP, $\mathrm{s}$ & $8.7(8.0-9.3)$ & $8.5(8.2-9.9)$ & $8.9(8.0-9.9)$ & $n s, p=0.481$ \\
\hline VI, $n M / m i n$ & $116.7 \pm 42.6^{*}$ & $107.4 \pm 48.8$ & $90.7 \pm 41.9 *$ & ${ }^{*} p=0.002$ \\
\hline \multicolumn{5}{|l|}{$0 \mathrm{pM} \mathrm{TF}$} \\
\hline LT, S & $11.0(9.8-12.6)$ & $11.8(10.2-12.5)$ & $11.5(9.5-13.5)$ & ns \\
\hline ETP, nM.min & $1452 \pm 354^{\star}$ & $1379 \pm 305^{*}$ & $1331 \pm 295$ & ${ }^{*} p<0.001$ \\
\hline Peak, $n M$ & $355(308-390)^{*}$ & $335(281-386) \dagger$ & $315(264-347)^{\star}+$ & $\begin{array}{l}{ }^{*} p=0.003 \\
t p=0.016\end{array}$ \\
\hline TTP, $s$ & $13.0(12.0-15.0)$ & $13.8(12.3-15.2)$ & $13.9(11.5-15.8)$ & $n s, p=0.787$ \\
\hline $\mathrm{VI}, n M / \min$ & $162.7 \pm 48.1^{\star}$ & $152.4 \pm 60.0 \dagger$ & $133.0 \pm 43.1^{* \dagger}$ & $\begin{array}{l}{ }_{*} p=0.003 \\
t p=0.008\end{array}$ \\
\hline
\end{tabular}

Of note, other global assays for plasmatic coagulation, the prothrombin time (PT) and activated partial thromboplastin time (aPTT), did not show any differences between the 3 time points (Table S2). CRP correlated significantly with the ETP (Spearman $r$ between 0.244 and 0.315 ), peak ( $r$ between 0.361 and 0.405 ) and velocity index ( $r$ between 0.364 and 0.450) of TG stimulated with 0, 1 and 5 pM (Table S3). In line with this, patients with CRP>10 mg/L had a significantly elevated peak, ETP and velocity index compared to those without systemic inflammation (Fig. S1).

Data are presented as mean \pm SD for normally distributed variables and median (25th-75th quartile) for non-parametric variables. * $+\dagger$ and $\S$ indicate the values that are significantly different from each other $(n=32)$. Abbreviations: AE-COPD, acute exacerbation of COPD; TF, tissue factor; LT, lagtime; ETP, endogenous thrombin potential; 
TTP, time to thrombin peak; VI, velocity index; ns, non-significant at the $p=0.05$ level. Fibrinogen was significantly elevated during AE-COPD (mean $4.8 \pm$ SD 1.5) compared to day 3 of treatment (mean $4.2 \pm$ SD 1.3) (Table S2). Levels of FVIII were highest at day 3 of treatment (median 198, IQR 87) compared to onset AE-COPD (median 185, IQR 91) and convalescence (median 153, IQR 84). Fibrinogen and FVIII levels correlated with CRP (Spearman $r=0.722$ and 0.339, respectively, both $p<0.001$ ) and were significantly increased in patients with signs of systemic inflammation (fibrinogen mean 5.4, SD $1.2 \mathrm{~g} / \mathrm{L}$; FVIII median 154\%, IQR 78\%) compared to patients without systemic inflammation (fibrinogen mean 3.6, SD 0.7 g/L; FVIII median 205\%, IQR 78\%). D-dimer levels, a marker of in vivo coagulation activation, were not different between the 3 time points (Table S2).

\section{Fibrinolysis rate is decreased in patients with systemic inflammation}

To study the influence of an AE-COPD on fibrinolysis we measured the clot lysis time $(\mathrm{CLT})$ at all three study time points. At day 3 of in-hospital treatment, the clot lysis time was significantly longer (median 22.9 min, IQR 9.4 min) compared to during AE-COPD (20.8 min, IQR 8.7 min, $p=0.001$ ) (Fig. S2, panel A; Table S2). At convalescence, the CLT returned back to the AE-COPD level (median 20.4, IQR 7.6, $p=0.001$ ). A significant, positive correlation between CLT and CRP levels ( $r=0.297, p=0.01$ ) was observed. Moreover, during AE-COPD, patients with systemic inflammation (median 21.7 min, IQR 6.5) had significantly longer CLT than those without systemic inflammation (median 17.7 min, IQR 10.2, $p=0.013$ ) (Fig. S2, panel B).

\section{Clinical outcomes in relation to coagulation parameters}

Three patients died during hospital admission. Clinical outcomes could be determined for all other 49 patients. Three patients suffered a cardiovascular event during the study: during admission, one patient suffered a non-ST segment elevation myocardial infarction and one had acute Takotsubo cardiomyopathy, while after discharge one patient had an unprovoked pulmonary embolism. No remarkable baseline (AE-COPD) coagulation parameter values were observed in these 3 patients, and given the small number of events we refrained from further post-hoc analyses.

Twenty-one patients suffered from a relapse of AE-COPD within the study follow-up period. Post-hoc analysis showed that platelet hyperreactivity at baseline, i.e. at hospital admission, was significantly associated with AE-COPD relapse after hospital discharge: patients who would later relapse had significantly higher platelet allbß3 activation in response to TRAP (MFI 7.9, IQR 4.8, $p=0.001$ ), CRP-XL(MFI 44.8, IQR 21.0, $p=0.007$ ) and ADP (MFI 31.6, IQR 11.1, $\mathrm{p}=0.017$ ) at hospital admission compared to patients who did not have a relapse during the follow up (TRAP MFI 4.5, IQR 3.8; CRP-XL MFI 35.4, IQR 24.8; ADP MFI 23.6, IQR 10.9). 


\section{DISCUSSION}

Acute exacerbations of COPD are associated with a prothrombotic state that is characterized by both platelet- and endothelial activation, as well as by an increase in plasmatic coagulation potential. Moreover, activation of these coagulation parameters was associated with increased systemic inflammatory markers, suggesting that both inflammation and coagulation may drive the prothrombotic state in AE-COPD.

Despite the evidence that AE-COPDs are associated with an increased risk of arterial and venous thrombotic events $[1,26]$, there are surprisingly few studies directly linking AECOPD to the function and activation status of the coagulation system. In one prospective cohort study, Wedzicha et al. found that fibrinogen levels are elevated in stable COPD and further increase during an exacerbation [27]. Two smaller studies have shown an increase in circulating platelet-monocyte complexes (PMCS) during an AE-COPD $[16,17]$. Our study extends these results by showing that an AE-COPD is a "pan-thrombotic" state in which platelets, monocytes, the endothelium and plasmatic coagulation are all activated. Our finding that activated VWF is elevated during an AE-COPD is especially relevant since active VWF can directly interact with platelets, and is therefore highly thrombogenic. The observed simultaneous activation of the whole coagulation system supports the hypothesis that an AE-COPD itself is a thrombogenic trigger and thereby increases the risk of both arterial and venous thromboembolic events. Furthermore, measurements at day 3 of in-hospital treatment showed, on average, a beneficial effect of treatment, i.e. a decrease in prothrombotic markers such as TG and active VWF, likely as a result of strongly reduced inflammation. However, fibrinolysis, as measured by CLT was significantly prolonged at day 3. Further studies are needed to gain more insight into the effects of in-hospital exacerbation therapy on coagulation and fibrinolysis.

In addition, the coagulation system might potentially contribute to the occurrence of COPD exacerbations. Some studies have shown that even at baseline there might be some activation of the coagulation system in patients with stable COPD $[28,29]$. It is hypothesized that platelet- and endothelial activation might affect COPD progression and exacerbation rate by impairing microvascular pulmonary blood flow and maintaining inflammation in COPD [30]. In the MESA and SPIROMICS cohort studies chronic aspirin use was found to attenuate emphysema progression and was associated with a lower risk of exacerbations [31,32]. This is in line with our observation of increased VWF:Ag but stable VWFpp, which suggests chronic, rather than acute, endothelial activation [20]. Chronic endothelial activation in early adulthood has been associated with future emphysema development [33]. Moreover, we report significantly higher platelet reactivity during the index exacerbation in patients who later suffer from a recurrent exacerbation. Given the post-hoc nature of this analysis, the multiple comparisons, 
potential index event bias and risk of confounding, this finding should be interpreted very prudently.

We profiled thrombin generation to assess changes in plasmatic coagulation during an AE-COPD. Thrombin peak, ETP and velocity of thrombin generation were significantly increased during AE-COPD, while D-dimer levels, a marker of in vivo coagulation activation, did not change. In addition, the time until clot formation, reflected by the aPTT, PT and TG lagtime, were comparable at all time points. Together, this implies that the increased thrombin generation potential mainly serves to stabilize clots. Increased peak TG has been associated with subsequent risk of venous thromboembolism (VTE), independent of established VTE risk factors [34]. TG peak, ETP and velocity significantly correlated with inflammation, possibly through the inflammatory cytokine IL-6, which stimulates fibrinogen and FVIII release $[35,36]$. Elevated FVIII and fibrinogen levels during AE-COPD contribute to the increased TG [27, 37]. Moreover, Mac-1 on activated monocytes catalyzes the conversion of FX to FXa, which also boosts TG [38]. Of note, the high TG peak and velocity index in the absence of added TF indicate that in vivo processes increase the availability of TF. These processes are likely linked to systemic inflammation, as IL-6 and CRP induce TF expression on monocytes, and apoptosis of several cell types increases circulating TF-bearing microparticles [39]. Together, these results underscore the close relationship between inflammation and coagulation in the occurrence of thromboembolic events after an AE-COPD.

Contrary to our hypothesis, we did not find "in vivo" platelet activation and hyperreactivity as measured by allbß3 activation and P-selectin expression, either unstimulated or after stimulation with platelet agonists, respectively (Table 2). PMC formation may be a more sensitive marker of platelet activation, as circulating platelets rapidly shed surface P-selectin but continue to circulate in complex with monocytes [10]. The fact that we also found an increased mean platelet volume (Table S1), another surrogate marker of platelet activation, is in line with the increased PMC formation and supports that platelet activation did occur during AE-COPD. However, other mechanisms such as monocyte activation as the primary driver for PMC formation in COPD should be considered. We observed significantly increased monocyte Mac-1 expression during AE-COPD compared to convalescence, which correlated with PMC formation. Mac-1 expression is predominantly triggered by interaction with platelets through P-selectin-PSGL-1 binding but can also occur in response to inflammatory mediators such as CRP [38], which was significantly increased during AE-COPD and correlated positively with both Mac-1 expression and circulating PMCs. This would support the hypothesis that inflammationassociated monocyte activation plays a key role in the PMC formation during AE-COPD. Contrary to our hypothesis, we did not observe changes in the rate of fibrinolysis, measured as CLT, between AE-COPD and at convalescence. Though PAI-1 levels are 
known to be higher in COPD patients compared to healthy controls [40], PAI-1 levels did not rise during AE-COPD in a previous study [41]. Interestingly, AE-COPD patients with systemic inflammation had a prolonged clot lysis time compared to those without systemic inflammation. Inflammatory stimuli are known to induce increased expression of PAl-1 [42]. Although we did not directly measure PAI-1 levels, we hypothesize that the prolonged $C L T$ in AE-COPD patients with systemic inflammation may be (at least partly) explained by an inflammation-induced increase in PAI-1. The prolonged clot lysis time suggests that decreased fibrinolysis might additionally contribute to the prothrombotic state in this subgroup of AE-COPD patients with systemic inflammation.

Although the strengths of this study include the careful and comprehensive assessment of prothrombotic markers during an AE-COPD and after convalescence, several limitations should be discussed. First, our study sample was relatively small and loss-to-followup was larger than expected, which in part reflects the labor-intensive nature of such mechanistic studies. Nonetheless, due to the large changes in prothrombotic markers from exacerbation to convalescence, the study was still sufficiently powered to detect statistically significant changes. Of note, the large loss-to-follow-up could have potentially resulted in selection bias, although in post-hoc analyses we did not find any differences in outcome parameters between patients who withdrew themselves from the study $(n=17)$ or passed away ( $n=3)$, versus those who did complete the study $(n=32)$. Regrettably, we could not detect correlations between prothrombotic markers and the occurrence of cardiovascular outcome, nor could we find clinical risk factors for the increase in thrombotic markers. This is not surprising since our study was not set up and powered as such. Nevertheless, the large changes in thrombotic biomarkers seen in this study warrant future investigations into their relationship with the incidence of cardiovascular events. Our data suggest that active VWF, PMCs, and TG could be candidate biomarkers in such future studies. Of note, 37 out of 52 patients were GOLD III or IV, hence our findings should not be extrapolated to milder COPD, including exacerbations treated in primary care. Furthermore, the uniqueness of AE-COPD as a thrombogenic trigger should not be overestimated. Epidemiological studies in nonCOPD populations have yielded similar results, demonstrating an increased cardiovascular risk following acute infections [43]. Since COPD patients are more susceptible to infections, it might not be an intrinsic characteristic of COPD patients to develop thrombosis, but rather a multitude of infectious insults that initiates the thrombotic cascade. In addition, we included 3 time-points in our study, whereas longer, sequential time points would have provided more insight into the course of the prothrombotic changes seen during and after an AE-COPD and the subsequent risk of CVD. Finally, we applied a Bonferroni correction for multiple comparisons, which is known to be rather conservative, especially since the test statistics at each time point were not fully independent. This may have caused an increased risk of type II error/false negative rate, which was preferred over a high risk of type I error/false positive rate. 


\section{CONCLUSIONS}

In conclusion, elevated circulating PMCs, (active) VWF and thrombin generation, combined with decreased fibrinolysis, together contribute to the prothrombotic state of COPD patients during an acute exacerbation, particularly in patients with increased systemic inflammation. Moreover, platelet hyperreactivity was associated with exacerbation relapse. These findings signify that AE-COPDs are "pan-thrombotic" events, but also suggest a reciprocal relationship in which increased platelet hyperreactivity may contribute to recurrent COPD exacerbations. The current study may therefore provide a basis for future studies with the aim to predict which AE-COPD patients have the highest risk of developing a cardiovascular event.

\section{Funding}

The authors gratefully acknowledge the financial support of the Gelre Hospitals Science Fund (Gelre Ziekenhuizen Wetenschapsfonds) for this study.

\section{Credit authorship contribution statement}

Lisa N. van der Vorm: Conceptualization, Methodology, Investigation, Formal analysis, Data curation, Writing - original draft, Visualization, Funding acquisition, Project administration. Li Li: Methodology, Investigation, Formal analysis, Writing - review \& editing. Dana Huskens: Methodology, Investigation, Formal analysis, Writing - review \& editing, Supervision. Janine J.J. Hulstein: Conceptualization, Writing - review \& editing, Supervision. Mark Roest: Conceptualization, Writing - review \& editing, Supervision. Philip G. de Groot: Conceptualization, Writing - review \& editing, Supervision. Hugo ten Cate: Conceptualization, Writing - review \& editing, Supervision. Bas de Laat: Conceptualization, Resources, Writing review \& editing, Supervision. Jasper A. Remijn: Methodology, Resources, Writing - review \& editing, Supervision. Sami O. Simons: Conceptualization, Methodology, Investigation, Formal analysis, Writing - review \& editing, Supervision, Funding acquisition, Project administration, All authors approved the final version of this manuscript.

\section{Declaration of competing interest}

The authors declare that they have no known competing financial interests or personal relationships that could have appeared to influence the work reported in this paper.

\section{Acknowledgments}

The authors would like to express their gratitude to all colleagues of the Department of Clinical Chemistry and Hematology of the Gelre Hospitals Apeldoorn for their indispensable help with blood drawings and their technical support in the laboratory. Furthermore, we would like to thank all colleagues of the Department of Respiratory medicine of the Gelre Hospitals Apeldoorn for their help during the inclusion phase of the study. 


\section{REFERENCES}

1. Anthonisen NR, Connett JE, Enright PL, Manfreda J: Hospitalizations and mortality in the Lung Health Study. American journal of respiratory and critical care medicine 2002, 166(3):333-339.

2. Donaldson GC, Hurst JR, Smith CJ, Hubbard RB, Wedzicha JA: Increased risk of myocardial infarction and stroke following exacerbation of COPD. Chest 2010, 137(5):1091-1097.

3. Maclay JD, McAllister DA, Macnee W: Cardiovascular risk in chronic obstructive pulmonary disease. Respirology 2007, 12(5):634-641.

4. Rothnie KJ, Yan R, Smeeth L, Quint JK: Risk of myocardial infarction (MI) and death following MI in people with chronic obstructive pulmonary disease (COPD): a systematic review and meta-analysis. BMJ open 2015, 5(9):e007824.

5. Papi A, Bellettato CM, Braccioni F, Romagnoli M, Casolari P, Caramori G, Fabbri LM, Johnston SL: Infections and airway inflammation in chronic obstructive pulmonary disease severe exacerbations. American journal of respiratory and critical care medicine 2006, 173(10):1114-1121.

6. Thomsen M, Ingebrigtsen TS, Marott JL, Dahl M, Lange P, Vestbo J, Nordestgaard BG: Inflammatory biomarkers and exacerbations in chronic obstructive pulmonary disease. Jama 2013, 309(22):23532361.

7. Malerba M, Clini E, Malagola M, Avanzi GC: Platelet activation as a novel mechanism of atherothrombotic risk in chronic obstructive pulmonary disease. Expert Rev Hematol 2013, 6(4):475483.

8. Davì G, Patrono C: Platelet activation and atherothrombosis. The New England journal of medicine 2007, 357(24):2482-2494.

9. Wang Y, Sakuma M, Chen Z, Ustinov V, Shi C, Croce K, Zago AC, Lopez J, Andre P, Plow E et al: Leukocyte engagement of platelet glycoprotein Ibalpha via the integrin Mac-1 is critical for the biological response to vascular injury. Circulation 2005, 112(19):2993-3000.

10. Michelson AD, Barnard MR, Krueger LA, Valeri CR, Furman MI: Circulating monocyte-platelet aggregates are a more sensitive marker of in vivo platelet activation than platelet surface P-selectin: studies in baboons, human coronary intervention, and human acute myocardial infarction. Circulation 2001, 104(13):1533-1537.

11. Furman MI, Barnard MR, Krueger LA, Fox ML, Shilale EA, Lessard DM, Marchese P, Frelinger AL, Goldberg RJ, Michelson AD: Circulating monocyte-platelet aggregates are an early marker of acute myocardial infarction. Journal of the American College of Cardiology 2001, 38(4):1002-1006.

12. Furman MI, Benoit SE, Barnard MR, Valeri CR, Borbone ML, Becker RC, Hechtman HB, Michelson AD: Increased platelet reactivity and circulating monocyte-platelet aggregates in patients with stable coronary artery disease. Journal of the American College of Cardiology 1998, 31(2):352-358.

13. McCabe DJ, Harrison P, Mackie IJ, Sidhu PS, Purdy G, Lawrie AS, Watt H, Brown MM, Machin SJ: Platelet degranulation and monocyte-platelet complex formation are increased in the acute and convalescent phases after ischaemic stroke or transient ischaemic attack. British journal of haematology 2004, 125(6):777-787.

14. Ruggeri ZM: The role of von Willebrand factor in thrombus formation. Thromb Res 2007, 120 Suppl 
1(Suppl 1):S5-9.

15. Popović M, Smiljanić K, Dobutović B, Syrovets T, Simmet T, Isenović ER: Thrombin and vascular inflammation. Molecular and cellular biochemistry 2012, 359(1-2):301-313.

16. Maclay JD, McAllister DA, Johnston S, Raftis J, McGuinnes C, Deans A, Newby DE, Mills NL, MacNee W: Increased platelet activation in patients with stable and acute exacerbation of COPD. Thorax 2011, 66(9):769-774.

17. Munoz-Esquerre M, Ferreiro JL, Huertas D, Marcano AL, Lopez-Sanchez M, Roura G, Gomez-Hospital JA, Dorca J, Cequier A, Santos S: Impact of acute exacerbations on platelet reactivity in chronic obstructive pulmonary disease patients. Int J Chron Obstruct Pulmon Dis 2018, 13:141-148.

18. Polatli M, Cakir A, Cildag O, Bolaman AZ, Yenisey C, Yenicerioglu Y: Microalbuminuria, von Willebrand factor and fibrinogen levels as markers of the severity in COPD exacerbation. Journal of thrombosis and thrombolysis 2008, 26(2):97-102.

19. van der Vorm LN, Li L, Huskens D, Chayouâ W, Kelchtermans H, de Groot PG, Roest M, Remijn JA, de Laat B: Analytical characterization and reference interval of an enzyme-linked immunosorbent assay for active von Willebrand factor. PloS one 2019, 14(2):e0211961.

20. van Mourik JA, Boertjes R, Huisveld IA, Fijnvandraat K, Pajkrt D, van Genderen PJ, Fijnheer R: von Willebrand factor propeptide in vascular disorders: A tool to distinguish between acute and chronic endothelial cell perturbation. Blood 1999, 94(1):179-185.

21. Husebø GR, Gabazza EC, D’Alessandro Gabazza C, Yasuma T, Toda M, Aanerud M, Nielsen R, Bakke PS, Eagan TML: Coagulation markers as predictors for clinical events in COPD. Respirology 2021, 26(4):342-351.

22. Singh D, Agusti A, Anzueto A, Barnes PJ, Bourbeau J, Celli BR, Criner GJ, Frith P, Halpin DMG, Han M et al: Global Strategy for the Diagnosis, Management, and Prevention of Chronic Obstructive Lung Disease: the GOLD science committee report 2019. The European respiratory journal 2019, 53(5).

23. Huskens D, Roest M, Remijn JA, Konings J, Kremers RM, Bloemen S, Schurgers E, Selmeczi A, Kelchtermans $\mathrm{H}$, van Meel R et al: Strenuous exercise induces a hyperreactive rebalanced haemostatic state that is more pronounced in men. Thrombosis and haemostasis 2016, 115(6):1109-1119.

24. Bloemen S, Huskens D, Konings J, Kremers RM, Miszta A, de Laat B, Kelchtermans H: Interindividual Variability and Normal Ranges of Whole Blood and Plasma Thrombin Generation. The journal of applied laboratory medicine 2017, 2(2):150-164.

25. Kicken CH, van der Vorm LN, Zwaveling S, Schoenmaker E, Remijn JA, Huskens D, de Laat B: A Hypoxic Environment Attenuates Exercise-Induced Procoagulant Changes Due to Decreased Platelet Activation. TH Open 2019, 3(3):e216-e226.

26. Reilev M, Pottegard A, Lykkegaard J, Sondergaard J, Ingebrigtsen TS, Hallas J: Increased risk of major adverse cardiac events following the onset of acute exacerbations of COPD. Respirology 2019, 24(12):1183-1190.

27. Wedzicha JA, Seemungal TA, MacCallum PK, Paul EA, Donaldson GC, Bhowmik A, Jeffries DJ, Meade TW: Acute exacerbations of chronic obstructive pulmonary disease are accompanied by elevations of plasma fibrinogen and serum IL-6 levels. Thrombosis and haemostasis 2000, 84(2):210-215.

28. Aleva FE, Temba G, de Mast Q, Simons SO, de Groot PG, Heijdra YF, van der Ven A: Increased Platelet- 
Monocyte Interaction in Stable COPD in the Absence of Platelet Hyper-Reactivity. Respiration; international review of thoracic diseases 2018, 95(1):35-43.

29. Undas A, Jankowski M, Kaczmarek P, Sladek K, Brummel-Ziedins K: Thrombin generation in chronic obstructive pulmonary disease: dependence on plasma factor composition. Thromb Res 2011, 128(4):e24-28.

30. Wang M, Aaron CP, Madrigano J, Hoffman EA, Angelini E, Yang J, Laine A, Vetterli TM, Kinney PL, Sampson PD et al: Association Between Long-term Exposure to Ambient Air Pollution and Change in Quantitatively Assessed Emphysema and Lung Function. Jama 2019, 322(6):546-556.

31. Aaron CP, Schwartz JE, Hoffman EA, Angelini E, Austin JHM, Cushman M, Jacobs DR, Jr., Kaufman JD, Laine A, Smith LJ et al: A Longitudinal Cohort Study of Aspirin Use and Progression of Emphysemalike Lung Characteristics on CT Imaging: The MESA Lung Study. Chest 2018, 154(1):41-50.

32. Fawzy A, Putcha N, Aaron CP, Bowler RP, Comellas AP, Cooper CB, Dransfield MT, Han MK, Hoffman EA, Kanner RE et al: Aspirin Use and Respiratory Morbidity in COPD: A Propensity Score-Matched Analysis in Subpopulations and Intermediate Outcome Measures in COPD Study. Chest 2019, 155(3):519-527.

33. Wells JM, Colangelo LA, Sivarajan L, Thyagarajan B, Dransfield MT, Iribarren C, Reyfman PA, Jacobs DR, Washko GR, Kalhan R: Inflammation and endothelial activation in early adulthood are associated with future emphysema: the CARDIA Lung Study. The European respiratory journal 2019, 53(1).

34. Lutsey PL, Folsom AR, Heckbert SR, Cushman M: Peak thrombin generation and subsequent venous thromboembolism: the Longitudinal Investigation of Thromboembolism Etiology (LITE) study. Journal of thrombosis and haemostasis: JTH 2009, 7(10):1639-1648.

35. Amrani DL: Regulation of fibrinogen biosynthesis: glucocorticoid and interleukin-6 control. Blood coagulation \& fibrinolysis : an international journal in haemostasis and thrombosis 1990, 1(4-5):443446.

36. Stirling D, Hannant WA, Ludlam CA: Transcriptional activation of the factor VIII gene in liver cell lines by interleukin-6. Thrombosis and haemostasis 1998, 79(1):74-78.

37. Dielis AW, Castoldi E, Spronk HM, van Oerle R, Hamulyák K, Ten Cate H, Rosing J: Coagulation factors and the protein $\mathrm{C}$ system as determinants of thrombin generation in a normal population. Journal of thrombosis and haemostasis : JTH 2008, 6(1):125-131.

38. Altieri DC, Morrissey JH, Edgington TS: Adhesive receptor Mac-1 coordinates the activation of factor $X$ on stimulated cells of monocytic and myeloid differentiation: an alternative initiation of the coagulation protease cascade. Proceedings of the National Academy of Sciences of the United States of America 1988, 85(20):7462-7466.

39. Franco RF, de Jonge E, Dekkers PE, Timmerman JJ, Spek CA, van Deventer SJ, van Deursen P, van Kerkhoff L, van Gemen B, ten Cate $\mathrm{H}$ et al: The in vivo kinetics of tissue factor messenger RNA expression during human endotoxemia: relationship with activation of coagulation. Blood 2000, 96(2):554-559.

40. Polatli M, Yazici O, Kahvecioglu DA, Yenisey C, Abas Bl: The relation between plasminogen activator inhibitor-1 (PAI-1) and inflammatory markers in COPD: A possible risk factor of inflammation-induced thrombosis. EUROPEAN RESPIRATORY JOURNAL 2017, 50. 
41. Polosa R, Malerba M, Cacciola RR, Morjaria JB, Maugeri C, Prosperini G, Gullo R, Spicuzza L, Radaeli A, Di Maria GU: Effect of acute exacerbations on circulating endothelial, clotting and fibrinolytic markers in COPD patients. Internal and emergency medicine 2013, 8(7):567-574.

42. Kruithof EK: Regulation of plasminogen activator inhibitor type 1 gene expression by inflammatory mediators and statins. Thrombosis and haemostasis 2008, 100(6):969-975.

43. Cowan LT, Lutsey PL, Pankow JS, Matsushita K, Ishigami J, Lakshminarayan K: Inpatient and Outpatient Infection as a Trigger of Cardiovascular Disease: The ARIC Study. Journal of the American Heart Association 2018, 7(22):e009683. 


\section{SUPPLEMENTARY METHODS}

\section{Complete blood count and global clotting assays}

Blood cell counts were measured with a Cell Dyn Sapphire analyser (Abbott Diagnostics, Santa Clara, CA). Prothrombin time (PT), activated partial thromboplastin time (aPTT) and D-dimer were measured in PPP on a STA-R Max analyser (Diagnostica Stago, Leiden, the Netherlands).

\section{Von Willebrand Factor parameters}

VWF antigen (VWF:Ag) was measured on a STA-R Max coagulation analyzer (Stago, Leiden, the Netherlands). VWF ristocetin cofactor activity (VWF:RCo) was determined using the HemosIL ${ }^{\circledR}$ AcuStar chemiluminescence assay (IL-Werfen, Bedford, MA, USA). VWF propeptide (VWFpp) was measured with an ELISA (Sanquin, Amsterdam, The Netherlands). Results of the VWFpp and active VWF assay were normalized (\%) to normal pooled plasma (NPP) on the same plate.

\section{SUPPLEMENTARY FIGURES}
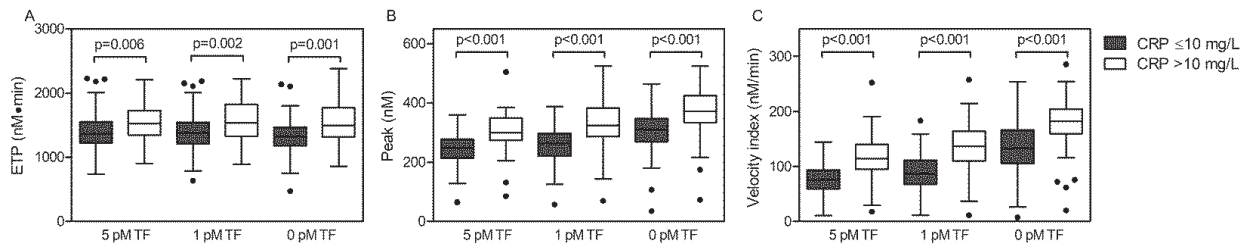

Figure S1. The effects of inflammation on thrombin generation. (A-C) ETP, peak and velocity index of TG in patients with (white) versus without (grey) systemic inflammation, defined as CRP>10 $\mathrm{mg} / \mathrm{L}$, for all tested concentrations of TF $(5,1$ and $0 \mathrm{pM})$. Centre lines represent the median, whiskers represent Tukey's whiskers. Corresponding p-values are indicated (Mann-Whitney $U$ test) $(n=52)$. Abbreviations: CRP, C-reactive protein; TF, tissue factor; ETP, endogenous thrombin potential. 

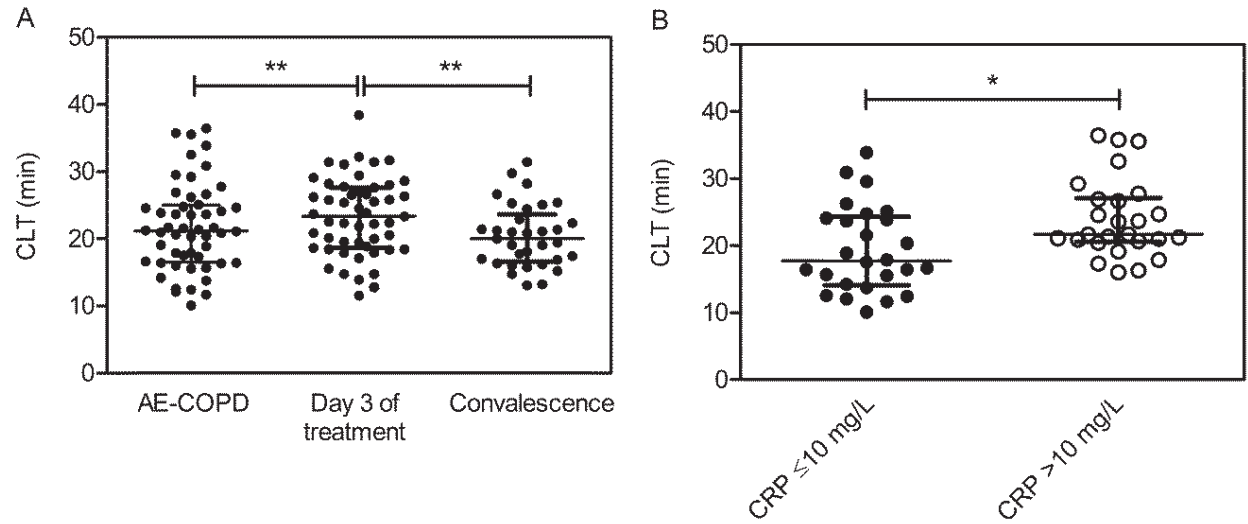

Figure S2. The effects of an AE-COPD and inflammation on fibrinolysis. (A) Clot lysis time was measured at exacerbation, day 3 of treatment and convalescence. The centre line represents the median, whiskers indicate the first and third quartiles $(n=32)$. (B) Clot lysis time in the presence (open circles) versus absence (closed circles) of systemic inflammation, defined as CRP>10 mg/L, in AE-COPD patients (at hospitalisation). The centre line represents the median, whiskers indicate the first and third quartiles ( $n=52){ }^{*}, p$-value $<0.05$ (Mann-Whitney $U$ test). Abbreviations: CRP, C-reactive protein; CLT, clot lysis time.

\section{SUPPLEMENTARY TABLES}

Table S1. Complete blood count parameters during and after AE-COPD.

\begin{tabular}{|c|c|c|c|c|c|}
\hline Parameter & AE-COPD & Day 3 of treatment & Convalescence & $p$-value & $\begin{array}{l}\text { Reference } \\
\text { values }\end{array}$ \\
\hline Leukocytes, $\times 10^{9} / \mathrm{L}$ & $11.9 \pm 3.5^{*}$ & $12.2 \pm 3.5 t$ & $10.0 \pm 3.4^{* \dagger}$ & $\begin{array}{l}{ }^{*} p=0.015 \\
t p=0.003\end{array}$ & $4-11$ \\
\hline Neutrophils, $\times 10^{9} / \mathrm{L}$ & $10.2 \pm 3.1^{*}$ & $8.8 \pm 3.4$ & $7.4 \pm 3.1^{\star}$ & ${ }^{*} p=0.001$ & $2-7.2$ \\
\hline Lymphocytes, $\times 10^{9} / \mathrm{L}$ & $0.8(0.5)^{* \dagger}$ & $2.4(1.4)^{* \S}$ & $1.7(1.0)+\S$ & $\begin{array}{l}{ }^{\star} p<0.001 \\
+p<0.001 \\
\S p=0.01\end{array}$ & $1-4$ \\
\hline Monocytes, $\times 10^{9} / \mathrm{L}$ & $0.6 \pm 0.3^{*}$ & $1.0 \pm 0.3^{*}+$ & $0.7 \pm 0.3 \dagger$ & $\begin{array}{l}{ }^{*} p<0.001 \\
t p<0.001\end{array}$ & $0.15-0.9$ \\
\hline Eosinophils, $\times 10^{9} / \mathrm{L}$ & $0.02(0.03)^{*}$ & $0.04(0.08) \dagger$ & $0.13(0.16)^{* \dagger}$ & $\begin{array}{l}{ }_{*}^{*} p<0.001 \\
t p<0.001\end{array}$ & $0-0.5$ \\
\hline Basophils, $\times 10^{9} / \mathrm{L}$ & $0.03(0.03)^{*}$ & $0.04(0.06)$ & $0.06(0.06)^{*}$ & ${ }^{*} p=0.001$ & $0-0.15$ \\
\hline Erythrocytes, $\times 10^{12} / \mathrm{L}$ & $4.5 \pm 0.4^{*}$ & $4.4 \pm 0.4^{* \dagger}$ & $4.6 \pm 0.4 \dagger$ & $\begin{array}{l}{ }^{*} p=0.005 \\
t p=0.001\end{array}$ & $3.8-6.0$ \\
\hline Platelets, $\times 10^{9} / \mathrm{L}$ & $279(108.5)$ & $281(132)$ & $272(120)$ & $n s, p=0.803$ & $150-400$ \\
\hline$M P V, f L$ & $8.9 \pm 1.7 *$ & $8.9 \pm 1.8 t$ & $8.2 \pm 1.3^{* \dagger}$ & $\begin{array}{l}{ }^{*} p<0.001 \\
t p<0.001\end{array}$ & $7.5-11.5$ \\
\hline
\end{tabular}

Data are presented as the mean \pm SD or median (IQR) for normally distributed and skewed continuous variables, respectively. ${ }^{*}, \dagger$ and $\S$ indicate the values that are significantly different from each other in Wilcoxon signed-rank tests, whereas "ns" and the corresponding p-value indicates the result of the Friedman test if not statistically significant ( $n=32)$. Abbreviations: $n s$, non-significant; MPV, mean platelet volume. 
Table S2. Global coagulation assays and coagulation factors

\begin{tabular}{|c|c|c|c|c|c|}
\hline Parameter & AE-COPD & Day 3 of treatment & Convalescence & p-value & Ref. values \\
\hline \multicolumn{6}{|c|}{ Global coagulation assays } \\
\hline $\mathrm{PT}, \mathrm{s}$ & $13.3(1.13)$ & $12.8(1.9)$ & $13.1(0.9)$ & $n s, p=0.174$ & $11-14$ \\
\hline aPTT, s & $28(4.3)$ & $28(4.3)$ & $29(7.0)$ & $n s, p=0.135$ & $30-40$ \\
\hline \multicolumn{6}{|c|}{ Markers of inflammation/acute phase proteins } \\
\hline CRP, $m g / L$ & $40.6 \pm 64.8 * \S$ & $20.8 \pm 26.9 *+$ & $6.6 \pm 12.6+\S$ & $\begin{array}{l}{ }^{*} p<0.001 \\
+p=0.005 \\
\S p<0.001\end{array}$ & $<10$ \\
\hline Fibrinogen, $g / L$ & $4.8 \pm 1.5^{\star}$ & $4.2 \pm 1.3^{*}$ & $4.2 \pm 1.0$ & ${ }^{*} p=0.001$ & $2-4$ \\
\hline \multicolumn{6}{|c|}{ Markers of in vivo coagulation } \\
\hline D-dimer, $n g / m L$ & $520(367)$ & $530(503)$ & $510(360)$ & $n s, p=0.531$ & $<500$ \\
\hline \multicolumn{6}{|l|}{ VWF and FVIII } \\
\hline VWF:Ag, \% & $218(113)^{*}$ & $217(126) \dagger$ & $182(110)^{\star \dagger}$ & $\begin{array}{l}{ }^{*} p=0.009 \\
t p<0.001\end{array}$ & $50-150$ \\
\hline WWF:RCo, \% & $156.7(88.8)$ & $158.3(64.4)^{* \dagger}$ & $135.1(71.4)^{* \dagger}$ & $\begin{array}{l}* p=0.009 \\
t p=0.002\end{array}$ & $50-150$ \\
\hline Active VWF, $\%$ of NPP & $151(48)^{\star} \S$ & $142(32)^{* \dagger}$ & $125(27)+\S$ & $\begin{array}{l}{ }^{*} p<0.001 \\
+p<0.001 \\
\S p<0.001\end{array}$ & $92-155$ \\
\hline WWFpp, \% of NPP & $123 \pm 48$ & $118 \pm 38$ & $109 \pm 42$ & $n s, p=0.123$ & $73-189$ \\
\hline FVIII:C, \% & $185(91)^{\star}$ & $198(87)^{* \dagger}$ & $153(84) \dagger$ & $\begin{array}{l}{ }^{*} p=0.001 \\
+p<0.001\end{array}$ & $50-150$ \\
\hline \multicolumn{6}{|l|}{ Fibrinolysis } \\
\hline $\mathrm{CLT}$, min & $20.8(8.7)^{\star}$ & $22.9(9.4)^{* \dagger}$ & $20.4(7.6) \dagger$ & $\begin{array}{l}{ }^{*} p=0.001 \\
t p=0.001\end{array}$ & \\
\hline
\end{tabular}

Data are presented as the mean \pm SD or median (IQR) for normally distributed and skewed continuous variables, respectively. ${ }^{*}, \dagger$ and $\S$ indicate the values that are significantly different from each other in Wilcoxon signed rank tests, whereas "ns" and corresponding p-values indicate the result of the Friedman test if not statistically significant $(n=32)$. Abbreviations: $n s$, non-significant; MPV, mean platelet volume, CLT, clot lysis time.

Table S3. Correlations between TG parameters and CRP.

\begin{tabular}{|c|c|c|}
\hline & \multicolumn{2}{|c|}{ Inflammation, CRP } \\
\hline & Spearman $r$ & $p$-value \\
\hline \multicolumn{3}{|l|}{$5 \mathrm{pM}$ TF } \\
\hline LT, S & 0.339 & $p<0.001$ \\
\hline ETP, nM.min & 0.244 & $p=0.008$ \\
\hline Peak, $n M$ & 0.405 & $p<0.001$ \\
\hline TTP, $s$ & -0.042 & $n s, p=0.250$ \\
\hline VI, $n M / m i n$ & 0.450 & $p<0.001$ \\
\hline \multicolumn{3}{|l|}{$1 \mathrm{pM}$ TF } \\
\hline LT, s & 0.158 & $n s, p=0.617$ \\
\hline ETP, nM.min & 0.277 & $p=0.002$ \\
\hline Peak, $n M$ & 0.388 & $p<0.001$ \\
\hline TTP,$S$ & -0.016 & $n s, p=0.463$ \\
\hline $\mathrm{VI}, n M / \min$ & 0.405 & $p<0.001$ \\
\hline \multicolumn{3}{|l|}{0 pM TF } \\
\hline LT, S & -0.009 & $n s, p=0.769$ \\
\hline ETP, nM.min & 0.315 & $p=0.001$ \\
\hline Peak, $n M$ & 0.361 & $p<0.001$ \\
\hline TTP, S & -0.041 & $n s, p=0.923$ \\
\hline $\mathrm{VI}, n M / \min$ & 0.367 & $p<0.001$ \\
\hline
\end{tabular}

Abbreviations: TF, tissue factor; LT, lagtime; ETP, endogenous thrombin potential; TTP, time to thrombin peak; VI, velocity index; ns, non-significant with corresponding p-values ( $n=32)$. 



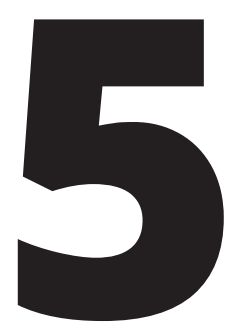

\section{Flow cytometric analysis of platelet function to detect high on-treatment residual platelet reactivity in patients on dual antiplatelet therapy}


Dear editors,

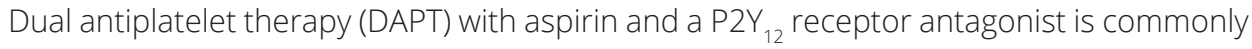
prescribed as secondary prevention for patients after percutaneous angioplasty with stent implantation, and it was shown to effectively reduce future ischemic events in patients with atherosclerotic cardiovascular disease[1]. Clopidogrel is the most frequently prescribed P2Y $Y_{12}$ antagonist, though variability in its pharmacokinetic and pharmacodynamic effect. It is an orally administered prodrug that needs metabolization in the liver to exert its antiplatelet effect. Differences in the extent and rapidity of this effect are partially due to genetic variations in the CYP2C19 enzyme that metabolizes the prodrug in its active metabolite[2]. Other factors that may contribute to the variable anti-platelet effect of clopidogrel are differences in absorption, smoking, drug interactions, and other patientrelated factors[3]. Several studies indicated high on-treatment residual platelet reactivity (HRPR) in patients on DAPT with higher risk of thrombotic events[1, 4]. Despite, there is discussion on measuring the in vitro effect of antiplatelet therapy.

In this study, approved by the ethical committee of the Ghent University Hospital, we assessed residual platelet reactivity in 42 patients receiving DAPT (median age 61 years, 33\% men). Citrated whole blood (BD Vacutainer, Becton Dickinson, Plymouth, UK) was collected. Light transmission aggregometry (LTA) was performed on a chrono-log model 700 (Chrono-log corporation, Havertown, US) according to the manufacturer's instructions and to the North American Consensus Guideline on platelet function testing (imprecision below 8\%)[5-8]. As alternative for LTA, to handle the increasing demand for antiplatelet drug monitoring in our laboratory, the whole blood platelet activation test (WB-PACT) was tested as it is a less time and sample volume consuming method[7]. WB-PACT was performed using a batch of tests strips stored at $-20^{\circ} \mathrm{C}$ with a total volume of $20 \mu \mathrm{l}$ consisting of no agonist or $2 \mu \mathrm{mol} / \mathrm{L}$ MesADP and $1.5 \mu \mathrm{lPE}$ conjugated anti-P-selectin, $2 \mu \mathrm{l}$ FITC-conjugated PAC-1 and $0.5 \mu \mathrm{l}$ APC-conjugated antiCD42b (BD Biosciences) in HEPES buffered saline (HBS, $10 \mathrm{mmol} / \mathrm{L}$ HEPES, $150 \mathrm{mmol} / \mathrm{L}$ $\mathrm{NaCl}, 1 \mathrm{mmol} / \mathrm{L} \mathrm{MgSO} 4,5 \mathrm{mmol} / \mathrm{L} \mathrm{KCL}, \mathrm{pH}$ 7.4). The assay was performed at $37^{\circ} \mathrm{C}$. Whole blood was 1:4 diluted in HEPES-buffered saline and $5 \mu \mathrm{l}$ was added to each test. After an incubation of 20 minutes the reaction was stopped by adding $250 \mu$ fixation solution (137 mmol/L NaCl, $2.7 \mathrm{mmol} / \mathrm{L} \mathrm{KCl}, 1.12 \mathrm{mmol} / \mathrm{L} \mathrm{NaH} 2 \mathrm{PO} 4,1.15 \mathrm{mmol} / \mathrm{L}$ KH2PO4, $10.2 \mathrm{mmol} / \mathrm{L} \mathrm{Na2HPO} 4,4 \mathrm{mmol} / \mathrm{L}$ EDTA, 0.5\% formaldehyde). FACS Canto ${ }^{\mathrm{TM}}$ II (BD Biosciences) was used to analyze the samples. Platelets were discriminated form other cells using the forward and sideward scatter pattern and gating on the CD42b positive cells. Fluorescent intensity in the FITC gate and PE channels were determined and results were expressed as median fluorescent intensity (MFI) corrected for the MFI of blood to which no platelet agonist was added. Intra-assay variation of the test is $5.3 \%$ and $13.3 \%$ for allbß3 activation and P-selectin, respectively. 
In 56 healthy donors (median age of 25, 44\% men) recruited in the hospital and not taking any medication that influences haemostasis, allbß3 activation and P-selectin expression was quantified and the $2.5^{\text {th }}, 10^{\text {th }}, 25^{\text {th }}$ and $50^{\text {th }}$ percentiles were determined. The allbB3 activation and P-selectin expression in men and women on DAPT are shown in Figure 1A. For LTA, maximal aggregation, disaggregation and prolongation of the lagtime in response to ADP was measured. The scoring system for both tests is shown in Figure 1B. There was a strong correlation between the WB-PACT platelet score in response to MesADP and the LTA platelet score in response to ADP with a Spearman correlation coefficient of 0.65 ( $P<0.001$, Figure 2$)$.

A
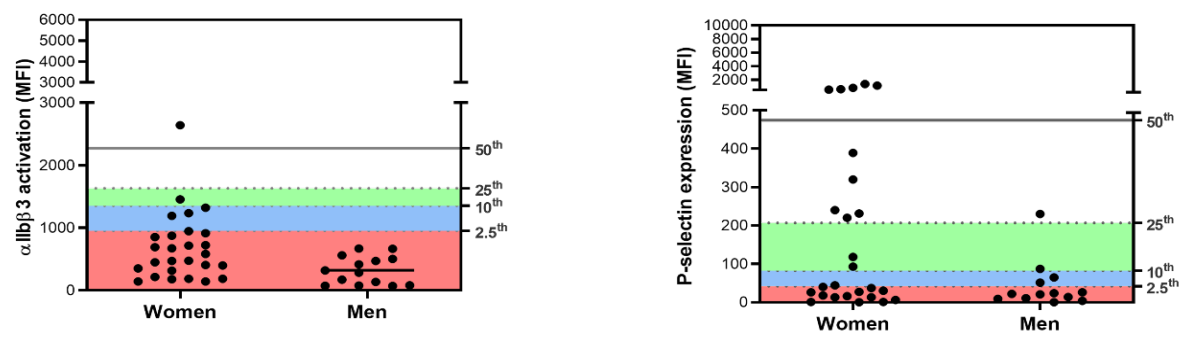

B

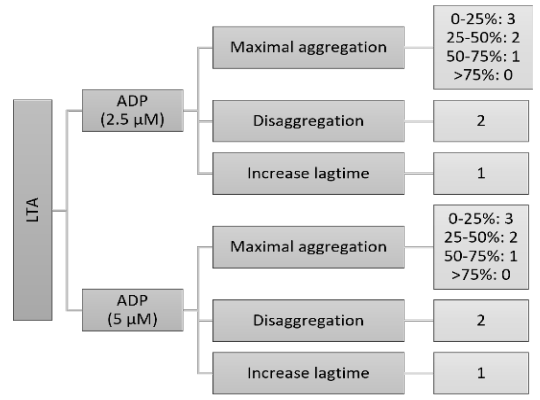

Figure 1. For WB-PACT, both allbB3 receptor activation (panel A left) and P-selectin expression (panel A right) in response to MeSADP was determined in blood of patients on dual antiplatelet therapy (black dots). The $50^{\text {th }}$ (median), $25^{\text {th }}, 10^{\text {th }}$ and $2.5^{\text {th }}$ percentiles, of the healthy population are indicated. For each patient on DAPT, a score for each parameter was given (panel B left). For LTA, maximal aggregation, disaggregation, and prolongation of the lagtime was scored (panel B right).

To study the agreement between both tests, patients on DAPT with at least one parameter lower than the $10^{\text {th }}$ percentile measured with WB-PACT and at least one value deviating from the normal range ( $<50 \%$ maximal aggregation, prolonged lagtime and/or disaggregation $>20 \%$ ) measured with LTA were considered sensitive for DAPT. If the criteria were not met, patients were recognized as HRPR. In total, 40 out of 42 
patients (95\%) on DAPT showed an agreement between MesADP-triggered WB-PACT and ADP-triggered LTA (Cohen's kappa $(\mathrm{K})=0.48, \mathrm{P}=0.002)$. This was a better agreement than the agreement described earlier between LTA and AggreGuide A-100 ( $K=0.019)$ [3]. Reduced platelet reactivity was measured in 39 patients on DAPT, and one patient was HRPR according to both WB-PACT and LTA. In addition, there was one patient that was HRPR according to WB-PACT but not according to LTA and one patient according to LTA but not according to WB-PACT.

Data on the application of WB-PACT in the measurement of platelet reactivity of patients on DAPT therapy are scare. A comparable correlation between aggregometry and flow cytometry for the assessment of ADP-induced platelet reactivity in patients on DAPT was reported by Gremmel et al[9]. Our data suggest that the WB-PACT may be a feasible alternative for LTA.

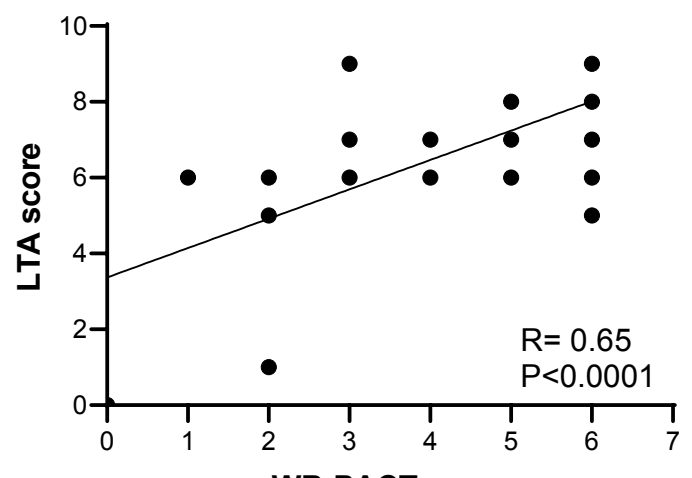

WB-PACT score

Figure 2. Correlation of WB-PACT with LTA for determining platelet function in patients on DAPT. The Spearman correlation coefficient and $\mathrm{P}$ value are depicted.

\section{Funding}

L.L. was supported by the China Scholarship Council (CSC) via the State Scholarship Fund (File No. 201706230245).

\section{Acknowledgements}

We would like to thank Taissa Adams, Diëgo Arnoe, Julie De Leenheer, An De Saar, Michael Luypaert and Esha Wauters for their technical support. 


\section{REFERENCES}

1. Collaborative meta-analysis of randomised trials of antiplatelet therapy for prevention of death, myocardial infarction, and stroke in high risk patients. BMJ (Clinical research ed) 2002, 324(7329):7186.

2. Kubica A, Kozinski M, Grzesk G, Fabiszak T, Navarese EP, Goch A: Genetic determinants of platelet response to clopidogrel. Journal of thrombosis and thrombolysis 2011, 32(4):459-466.

3. Florin L, Devreese KMJ: Evaluation of AggreGuide A-100 for monitoring of antiplatelet therapy. International journal of laboratory hematology 2018, 40(6):e113-e116.

4. Matetzky S, Shenkman B, Guetta V, Shechter M, Beinart R, Goldenberg I, Novikov I, Pres H, Savion N, Varon D et al: Clopidogrel resistance is associated with increased risk of recurrent atherothrombotic events in patients with acute myocardial infarction. Circulation 2004, 109(25):3171-3175.

5. Hayward CP, Moffat KA, Raby A, Israels S, Plumhoff E, Flynn G, Zehnder JL: Development of North American consensus guidelines for medical laboratories that perform and interpret platelet function testing using light transmission aggregometry. American journal of clinical pathology 2010, 134(6):955-963.

6. Le Quellec S, Bordet JC, Negrier C, Dargaud Y: Comparison of current platelet functional tests for the assessment of aspirin and clopidogrel response. A review of the literature. Thrombosis and haemostasis 2016, 116(4):638-650.

7. Heireman L, Monteyne T, Hemelsoet D, van Schaik RHN, Devreese KMJ: Search for a practical approach for detection of clopidogrel resistance: Comparison of light transmission aggregometry and INNOVANCE(R) PFA P2Y cartridge and correlation with CYP2C19 variants. International journal of laboratory hematology 2020, 42(4):e189-e191.

8. Monteyne T, Heireman L, Hemelsoet D, van Schaik RHN, Devreese KMJ: Is monitoring of antiplatelet therapy by light transmission aggregometry dependent on instrument and reagent used? International journal of laboratory hematology 2021.

9. Gremmel T, Koppensteiner R, Panzer S: Comparison of Aggregometry with Flow Cytometry for the Assessment of Agonists -Induced Platelet Reactivity in Patients on Dual Antiplatelet Therapy. PloS one 2015, 10(6):e0129666. 



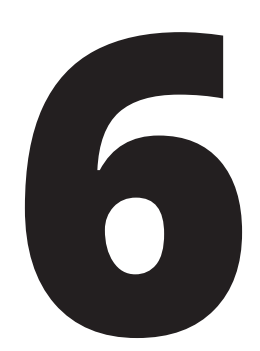

Flow cytometric analysis of platelet function to improve the recognition of thrombocytopathy

Dana Huskens

$\mathrm{Li} \mathrm{Li}$

Lisa Florin

Pieter De Kesel

Bas de Laat

Mark Roest

Katrien M.J. Devreese

Thromb Res. Oct 2020;194:183-189. 


\section{ABSTRACT}

\section{Introduction}

Light transmission aggregometry (LTA) is the gold standard for diagnosing bleeding disorders. Although LTA is laborious, requires large volumes of blood and is relatively insensitive to small changes in platelet function, there is still no competing alternative approach to replace LTA for the diagnosis of platelet bleeding disorders

\section{Materials and Methods}

This study investigates the correlation between flow cytometry-based whole blood platelet activation test (WB-PACT) and LTA and whether WB-PACT is of additional value for the identification of bleeding disorders. In total, 161 patients with suspected bleeding diathesis were tested.

\section{Results}

A correlation of 0.41 between LTA and WB-PACT was found, and there was agreement between tests in $62 \%$ of cases ( $K=0.23$ ). The WB-PACT is of additional value to LTA to detect platelet function disorders (PFD) as 10 patients with elevated bleeding score (BS) were detected with WB-PACT, 4 with LTA and 7 patients were positive with both tests. Interestingly, in contrast to LTA, WB-PACT has an additional option to detect VWF disfunctions.

\section{Conclusion}

WB-PACT may have added value for the routine diagnostic work-up in patients who need to have platelet function tested. 


\section{INTRODUCTION}

The assessment of platelet (dys)function is required for the identification of mild bleeding disorders (MBDs) characterized by disproportional bleeding after trauma or surgery, menorrhagia, excessive and frequent mucocutaneous hemorrhage and easy bruising $[1,2]$.

Currently, light transmission aggregometry (LTA) is the 'gold standard' platelet function test to define suspected bleeding disorders. Although LTA is used for more than four decades in diagnostic laboratories, it still lacks sensitivity for mild platelet function defects (PFDs) and it does not predict the risk of bleeding complications among patients with various platelet function defects [3-8]. It has been shown that only $60 \%$ of patients with excessive clinical bleeding and a suspected underlying inherited PFD, had a demonstrable abnormality in platelet aggregation [9], while in patients with mild bleeding, approximately 40\% had a defect when assessed by LTA and serotonin and ATP release measurements $[9,10]$. Disadvantages of LTA are the poor standardization despite existing guidelines, the requirement of a considerable volume of a fresh blood sample and the fact that it is time and labor intensive and not applicable for samples with low platelet count [11-16]. Accordingly, alternative methods to measure platelet aggregation have been developed but despite potential advantages, none of these techniques competed with LTA with regard to bleeding diagnostics [16]. There is an urgent need for alternative methods to give complimentary information about mild platelet function defects.

The whole blood platelet activation test (WB-PACT), introduced in 1987 by Shattil et al. [17], is a flow cytometric approach that allows stimulation of platelets with multiple agonists, while platelet activation can be quantified with different types of activation markers of granule release, glycoprotein activation and phospholipid expression. Since many laboratories do not offer facilities for both aggregometry and flow cytometry, data regarding the agreement between both approaches are scarce and these data would be of great value to correctly interpret the results achieved with either of these two approaches. For the diagnosis of MBDs, recently, Boknäs et al. [18] suggested that flow cytometric analysis of platelet function could become a feasible alternative for LTA, while van Asten et al. [19] concluded that this approach has added value to LTA.

In this study, we compared LTA and WB-PACT for the detection of PFDs in patients with bleeding diathesis. 


\section{MATERIALS AND METHODS}

\section{Study population}

Platelet function by LTA and WB-PACT was measured in parallel in 161 patients investigated for bleeding diathesis and in 56 healthy controls. For the patients investigated for bleeding diathesis, a bleeding score was calculated using the ISTH-BAT (elevated BS: $>5$ in women, $>3$ in men and >2 in children) [20]. Our study protocol was approved by the ethical committee of the Ghent University Hospital. Citrated whole blood (BD Vacutainer, Becton Dickinson, Plymouth, UK) was collected and processed according to the recommendations for the standardization of LTA [16].

\section{Reagents}

Platelet agonists used for flow cytometric analysis of platelet function include the P2Y 12 agonists 2MeS-ADP (1624, Tocris), the protease activated receptor (PAR)-1 agonist thrombin receptor activator peptide (TRAP-6 (SFLLRN), H-2936; Bachem, Germany), the glycoprotein VI (GPVI) agonist collagen-related peptide (CRP, purchased from Professor Farndale, University of Cambridge, UK) [21]. Ristocetin was purchased from ABP Ltd (Germany). The monoclonal antibodies used were FITC-conjugated PAC1, directed against the activated allbB3 receptor, PE-conjugated anti-P-selectin (CD62P, clone AK4), APC-conjugated anti-GPIb (CD42b, clone HIP1) and APC-conjugated anti-allbß3 (CD41a), all purchased from BD Pharmingen (NJ, USA) and FITC-conjugated anti-VWF (CL7616F, Cedarlane, Burlington, USA).

Platelet agonists, used for LTA were ADP, epinephrine, arachidonic acid, ristocetin (Avant- Medical, The Netherlands), U46619 (Sigma Aldrich, Belgium) and collagen (Takeda Pharma, Austria).

\section{Whole blood platelet activation test (WB-PACT)}

Platelet activation tests for flow cytometric analysis were prepared as published earlier $[22,23]$. In short, test strips consisting of no agonist, $30 \mu \mathrm{mol} / \mathrm{L}$ TRAP, $5 \mu \mathrm{g} / \mathrm{ml} \mathrm{CRP}$ and 2 $\mu \mathrm{mol} / \mathrm{L}$ MeSADP were prepared in advance and stored at $-20^{\circ} \mathrm{C}$. Each reaction mixture with a total volume of $20 \mu \mathrm{l}$ consists of $2 \mu \mathrm{l} \mathrm{FITC-conjugated} \mathrm{PAC-1,1.5 \mu l} \mathrm{PE-conjugated}$ anti-P-selectin and $0.5 \mu \mathrm{APC}$-conjugated anti-CD42b with or without agonist in HEPESbuffered saline (HBS, $10 \mathrm{mmol} / \mathrm{L}$ HEPES, $150 \mathrm{mmol} / \mathrm{L} \mathrm{NaCl}, 1 \mathrm{mmol} / \mathrm{L} \mathrm{MgSO} 4,5 \mathrm{mmol} / \mathrm{L}$ $\mathrm{KCL}, \mathrm{pH}$ 7.4). In addition, test strips were prepared with no agonist, $1.2 \mathrm{mg} / \mathrm{ml}$ or 0.2 $\mathrm{mg} / \mathrm{ml}$ ristocetin in a reaction mixture of $2 \mu \mathrm{l}$ FITC-conjugated anti-VWF and $0.5 \mu \mathrm{APC}$ conjugated anti-CD41a in HEPES buffered saline, and stored at $-20^{\circ} \mathrm{C}$.

Whole blood was kept at RT for at least 30 min and maximum 4h. Test strips were thawed to $37^{\circ} \mathrm{C}$ and shortly centrifuged. Subsequently, whole blood was incubated at 
$37^{\circ} \mathrm{C}$ for 10 minutes and diluted $1: 4$ in pre-heated HEPES-buffered saline to minimize the formation of platelet aggregates. From this diluted blood, $5 \mu \mathrm{l}$ was added to each reaction mixture $(20 \mu$, final dilution $1: 20)$ and the tests were incubated for exactly 20 minutes at $37^{\circ} \mathrm{C}$. Reactions were stopped by adding $250 \mu \mathrm{l}$ fixation solution (137 $\mathrm{mmol} / \mathrm{L} \mathrm{NaCl}, 2.7 \mathrm{mmol} / \mathrm{L} \mathrm{KCl}, 1.12 \mathrm{mmol} / \mathrm{L} \mathrm{NaH} \mathrm{PO}_{4}, 1.15 \mathrm{mmol} / \mathrm{L} \mathrm{KH}_{2} \mathrm{PO}_{4^{\prime}} 10.2 \mathrm{mmol} / \mathrm{L}$ $\mathrm{Na}_{2} \mathrm{HPO}_{4}, 4 \mathrm{mmol} / \mathrm{L}$ EDTA, 0.5\% formaldehyde).

A FACS Canto ${ }^{\mathrm{TM}}$ II (BD Biosciences, Erembodegem, Belgium) was used to analyze the samples (supplemental Fig. 1). First, using the forward and sideward scatter pattern and gating on the CD42b or CD41a positive cells, platelets were discriminated from other cells. Fluorescent intensity in the FITC gate and PE gate was selected to determine activated allbß3 or VWF binding and P-selectin density, respectively and results are expressed as median fluorescent intensity (MFI) minus the MFI of the unstimulated cells. Single measurements were performed as intra-assay precision for this assay is below $5 \%$ with the exception of P-selectin expression in response to MesADP [22].

\section{Light transmission aggregometry}

Light transmission aggregometry (LTA) was performed on a chrono-log model 700 (Chrono-log corporation, Havertown, US) according to the manufacturer's instructions and to the North American Consensus Guideline on platelet function testing [15]. Platelet-rich citrate plasma was obtained after centrifugation (10 minutes, $180 \mathrm{~g} / \mathrm{min}$ ) (Hettich Universal 32; DJB Labcare, Buckinghamshire, UK). LTA curves were assessed after addition of $25 \mu \mathrm{L}$ of the agonists ADP (2.5 and $5 \mu \mathrm{mol} / \mathrm{L})$, collagen ( 2.5 and $5 \mu \mathrm{g} /$ $\mathrm{mL})$, ristocetin $(0.5$ and $1.5 \mathrm{mg} / \mathrm{mL})$, epinephrine $(10 \mu \mathrm{mol} / \mathrm{L})$, arachidonic acid $(A A)$ (1 $\mathrm{mmol} / \mathrm{L}$ ), and thromboxane A2 analogue (1 $\mu \mathrm{mol} / \mathrm{L})$ to $225 \mu \mathrm{L}$ platelet rich citrate plasma. Light transmission was recorded for 6 minutes and compared to platelet poor plasma (PPP). Aggregation was expressed as \% maximum aggregation compared to PPP, lagtime was expressed in seconds. Disaggregation was defined as the difference between maximal and final platelet aggregation after 6 minutes expressed as percentage and $>20 \%$ of disaggregation was considered significant. For LTA imprecision is below $8 \%$ for all parameters, ristocetin low concentration not considered because of the low maximal aggregation as expected in normal individuals. In routine setting, analyses are not performed in duplicate, but abnormal results are repeated as confirmation for decreased maximal aggregation or desaggregation.

\section{VWF antigen and VWF activity}

The HemosIL AcuStar VWF:Ag assay (VWF:Ag Acustar) and HemosIL AcuStar VWF:RCo assay (Instrumentation Laboratory) was performed on an ACL AcuStar ${ }^{\top M}$ (Instrumentation Laboratory) using magnetic particles coated with anti-VWF polyclonal antibodies (antigen detection) or Recombinant GPIba (rGPIba) (activity measurement)[24]. The assay was 
executed according to the instructions of the manufacturer.

\section{Scoring system platelet function tests}

The WB-PACT by flow cytometry quantifies allbß3 activation and P-selectin expression in response to MESADP, TRAP and CRP. Similarly, this test can be used to quantify VWF binding to platelets in response to ristocetin. The $2.5^{\text {th }}, 10^{\text {th }}$ and $25^{\text {th }}$ percentiles for WBPACT parameters were determined in 56 healthy donors to score the patients samples and the scoring system is shown in Fig. 1A. For LTA, maximal aggregation, disaggregation and prolongation of the lagtime in response to ADP, collagen, epinephrine, AA, a thromboxane A2 analogue and ristocetin was scored (Fig. 1B).

To study the agreement between both tests in patients investigated for bleeding diathesis, patients with at least one parameter lower than the $10^{\text {th }}$ percentile measured with WB-PACT or at least one parameter deviating from the normal range measured with LTA (<50\% maximal aggregation, prolonged lagtime and/or disaggregation $>20 \%$ ) were categorized as abnormal for PFD.

\section{Statistics}

Analyses were performed using the software packages SPSS version 23.0 and GraphPad Prism 6. Spearman rank correlations were used to test for correlations between the different methods. Agreement between the methods was assessed using two-bytwo analysis and Cohen's kappa statistic. P-values $<0.05$ were considered statistically significant. 

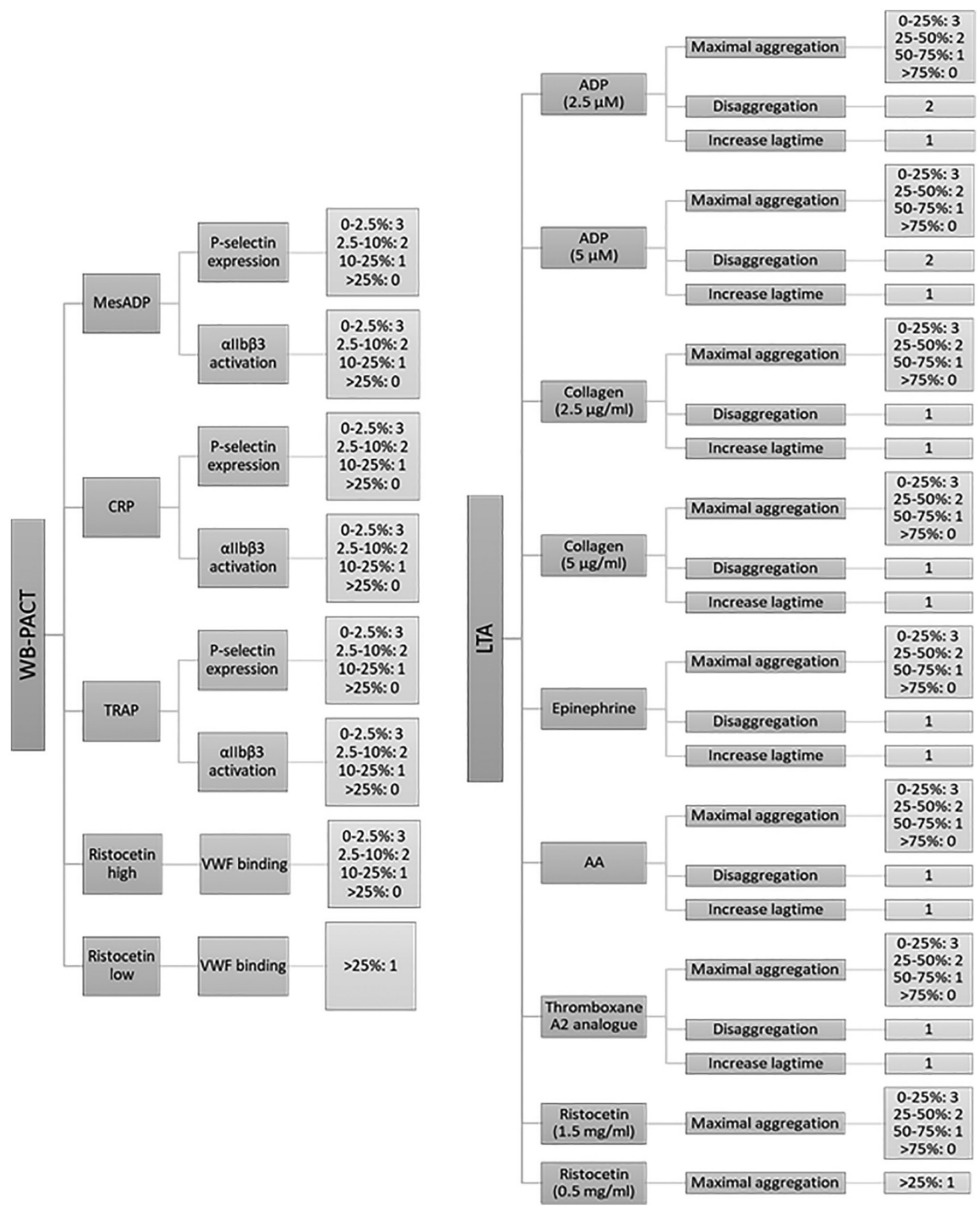

Figure 1. The WB-PACT was scored by first determining the 2.5 th, 10 th and 25 th percentiles for WB-PACT parameters in $\mathbf{5 6}$ healthy donors. In patients, if MFI was lower than the 2.5th percentile $(0-2.5 \%)$, between the 2.5th and 10th percentiles (2.5-10\%), between the 10th and 25th percentiles (10-25\%) or higher than the 25 th percentile $(>25 \%)$ a score of $3,2,1$ or 0 was given, respectively (panel A). For LTA, maximal aggregation, disaggregation and prolongation of the lagtime were scored. In patients, if maximum aggregation was between $0 \%$ and $25 \%$, between $25 \%$ and $50 \%$, between $50 \%$ and $75 \%$ or more than $75 \%$ as score of $3,2,1,0$ was given, respectively. If disaggregation was present, a score of 2 or 1 was given depending on the agonist and if an increase in lagtime was measured a score of 1 was given (panel B). Patients with at least one parameter lower than the 10th percentile measured with WB-PACT and at least one value deviating from the normal range measured with LTA were categorized as abnormal for PFD. 


\section{RESULTS}

\section{Patient characteristics, bleeding assessment and platelet function}

In total, 161 patients with a bleeding diathesis (median age 32 years) were included in the study (Table 1). A bleeding score was reported for 117 of 161 patients and an elevated BS was reported for 32\%, 18\% and 39\% of men, women and children, respectively.

Table 1. Baseline characteristics.

\begin{tabular}{lllll}
\hline & All & Men & Women & Children \\
\hline N & 161 & 31 & 100 & 30 \\
Age, years; median (IQR) & $32(20-51)$ & $54(27-61)$ & $35.5(24-50)$ & $9(4-13)$ \\
VWF:Ag (\%); median (IQR) & $93(68-122)$ & & & \\
VWF:Rco; median (IQR) & $87(64-120)$ & & & \\
BS & 117 & 22 & 72 & 23 \\
N of BS reported & 3 & 2 & 3 & 2 \\
median BS & 25 & 32 & 18 & 39 \\
$\%$ of elevated BS & & & & \\
\hline
\end{tabular}

$\mathrm{N}$, number of patients; IQR, interquartile ranges; BS, bleeding score; $\mathrm{N}$ of $\mathrm{BS}$ reported, number of patients interviewed to determine a bleeding score.

Platelet function was measured in response to MeSADP, CRP, TRAP and ristocetin and the extent of allbß3 activation, P-selectin expression and VWF binding to platelets is shown in Figure 2. In addition, platelet aggregation with LTA was measured according to general clinical practice [15]. For each agonist a score was calculated for both WB-PACT and LTA (Fig. 1, Table 2). In patients with expected bleeding diathesis, a low median score (0 or 1) was defined, for each platelet activation or aggregation agonist (Table 2). The distribution of the scores in the patients with bleeding diathesis is shown in supplemental Fig. 2.

More in detail, the binding of VWF to platelets detected by flow cytometry was more sensitive for diagnosing deviations in VWF concentration or function than ristocetin induced platelet agglutination measured with LTA (Fig. 3). In total, 29 patients were detected with abnormal VWF:Ag and/or VWF:RCo, and interestingly, 21/29 patients had an increased score for ristocetin (high concentration) induced binding of VWF to platelets (WB-PACT). More in detail, 8/29 patients were abnormal for VWF-binding to platelets (score $>1$ i.e. $<10^{\text {th }}$ percentile). In contrast, for LTA, only 1 out of 29 patients had a score different from zero (score 1 i.e. $<75 \%$ agglutination) for ristocetin induced agglutination of platelets. 
A

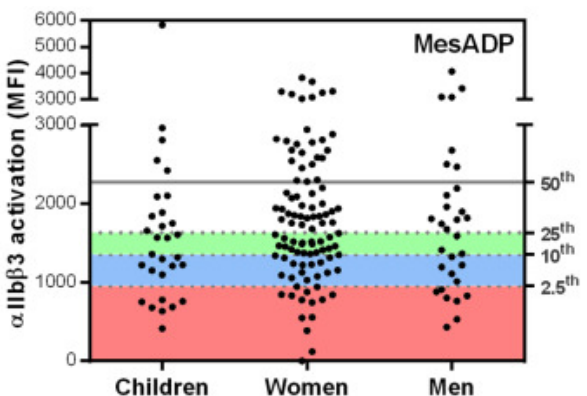

B

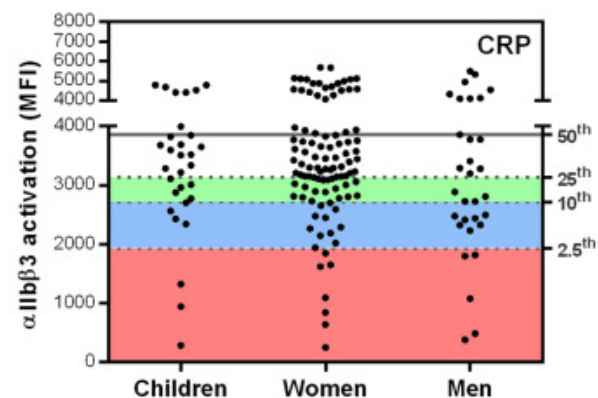

C

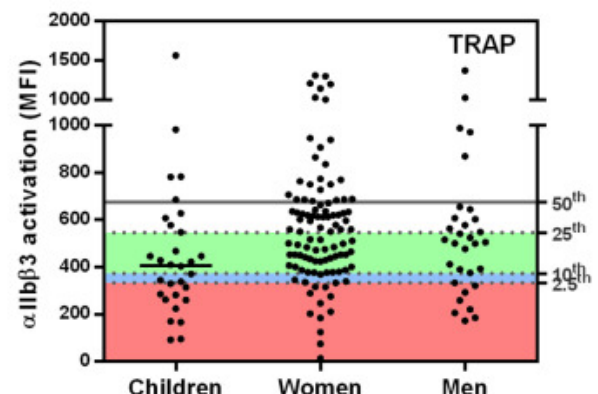

D

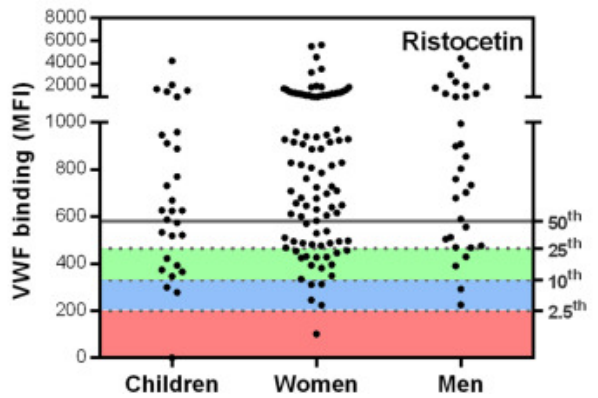

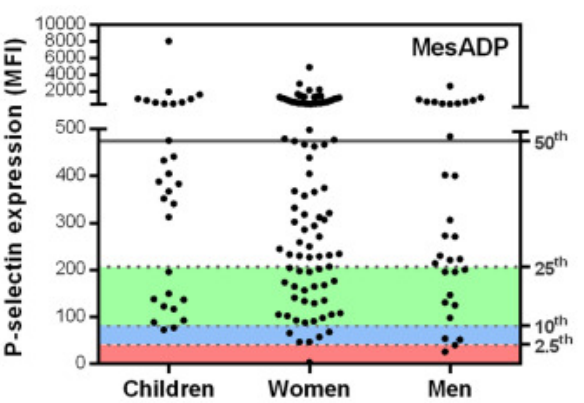
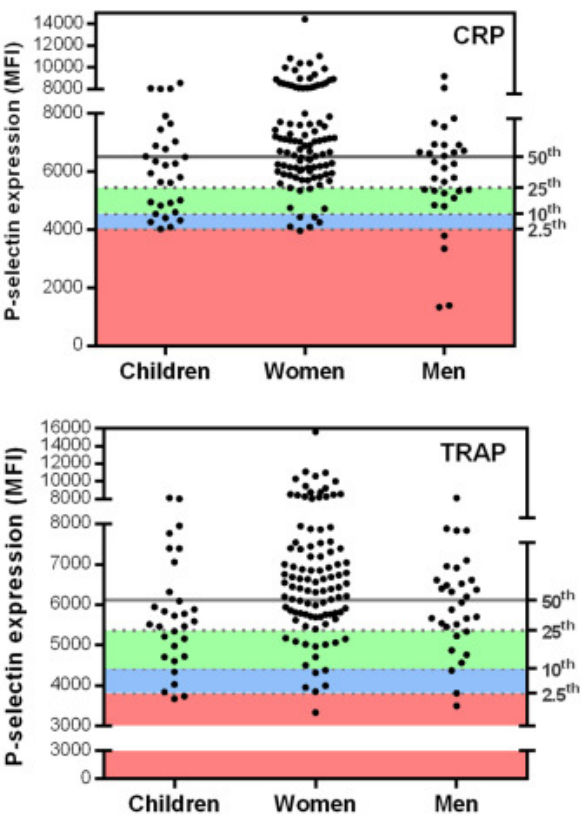

Figure 2. Both allb $\beta 3$ receptor activation (left panels) and P-selectin expression (right panels) in response to MeSADP (A), CRP (B) and TRAP (C) was determined in blood of patients with bleeding diathesis. In addition, VWF binding was determined in response to $1.5 \mathrm{mg} / \mathrm{ml}$ ristocetin (D) in the same population. The 50th (median), 25th, 10th and 2.5th percentiles, of the healthy population are indicated. If patients are between the 25th and 10th percentile (green), they received a score of one point for each agonist. If patients are between the 10th and 2.5th percentile (blue), they received a score of two points for each agonist. If patients are below the 2.5th percentile (red), they received a score of three points for each agonist. 


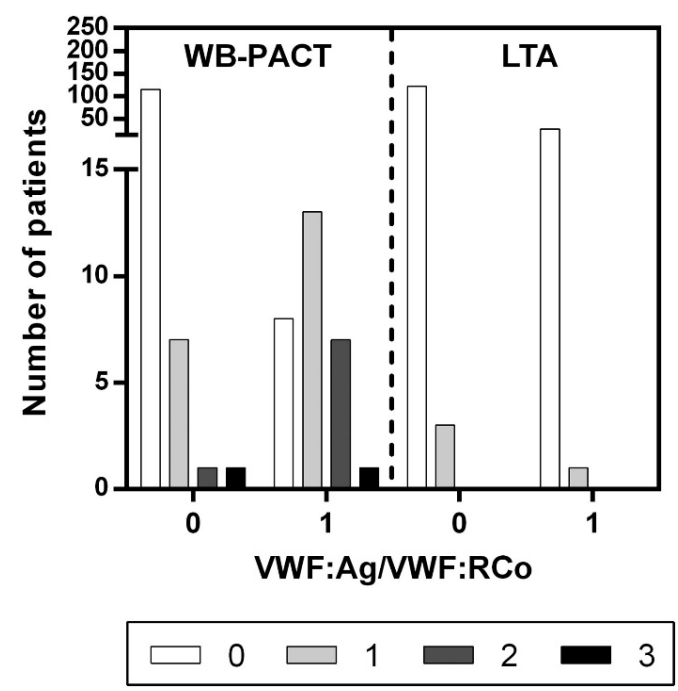

Figure 3. The scoring of the conventional methods to investigate VWF concentration and function was based on low VWF:Ag and/or low VWF:RCo (score 1) and normal VWF:Ag and VWF:RCo (score 0). The binding of VWF to platelets (WB-PACT) and platelet agglutination (LTA) in response to low and high concentrations of ristocetin is scored according to Fig. 1.

\section{Correlation and agreement between LTA and WB-PACT}

Furthermore, a Spearman correlation coefficient of 0.41 between LTA platelet activation score versus WB-PACT platelet activation score was observed (Fig. 4). Furthermore, for 99 of 161 patients (62\%) there was agreement between the tests $(K=0.23, P=0.003)$ with 46 patients (29\%) diagnosed with PFDs and 53 patients (33\%) without PFD. In total, 25 (15\%) and 37 (23\%) patients were diagnosed with PFD according to LTA and WB-PACT, respectively.

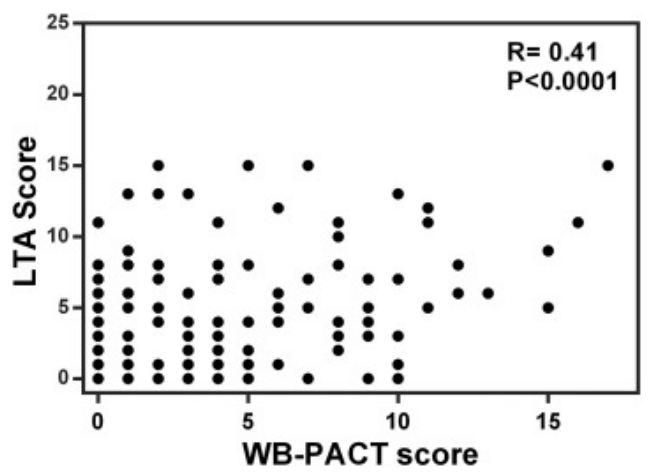

Figure 4. Correlation of WB-PACT with LTA for determining platelet function in patients suspected for bleeding diathesis. The Spearman correlation coefficient and $P$ value are depicted. 
In the group that was diagnosed with PFDs by both LTA and WB-PACT, 25\% of the patients had an elevated bleeding score (Fig. 5A). For patients with PFDs according to WB-PACT only and LTA only, an elevated BS was recorded for 33\% and $21 \%$ of the patients, respectively (Fig. 5B and C). In total, 8 ( 6 women and 2 men) out of 29 patients (28\%) with an elevated BS were not diagnosed with PACT nor LTA (Supplemental Fig. 3). In the group of children with elevated BS, 7 out of 9 children were detected with WB-PACT (78\%), while only 4 out of 9 children were detected with LTA (44\%). However, when combining both tests, all children with elevated BS were detected. Remarkably, in the group patients with a normal BS, 24\% were diagnosed with PFD with both tests, $17 \%$ with LTA, 23\% with WB-PACT, and $36 \%$ of patients were found negative for PFDs (supplemental Fig. 3B).

A

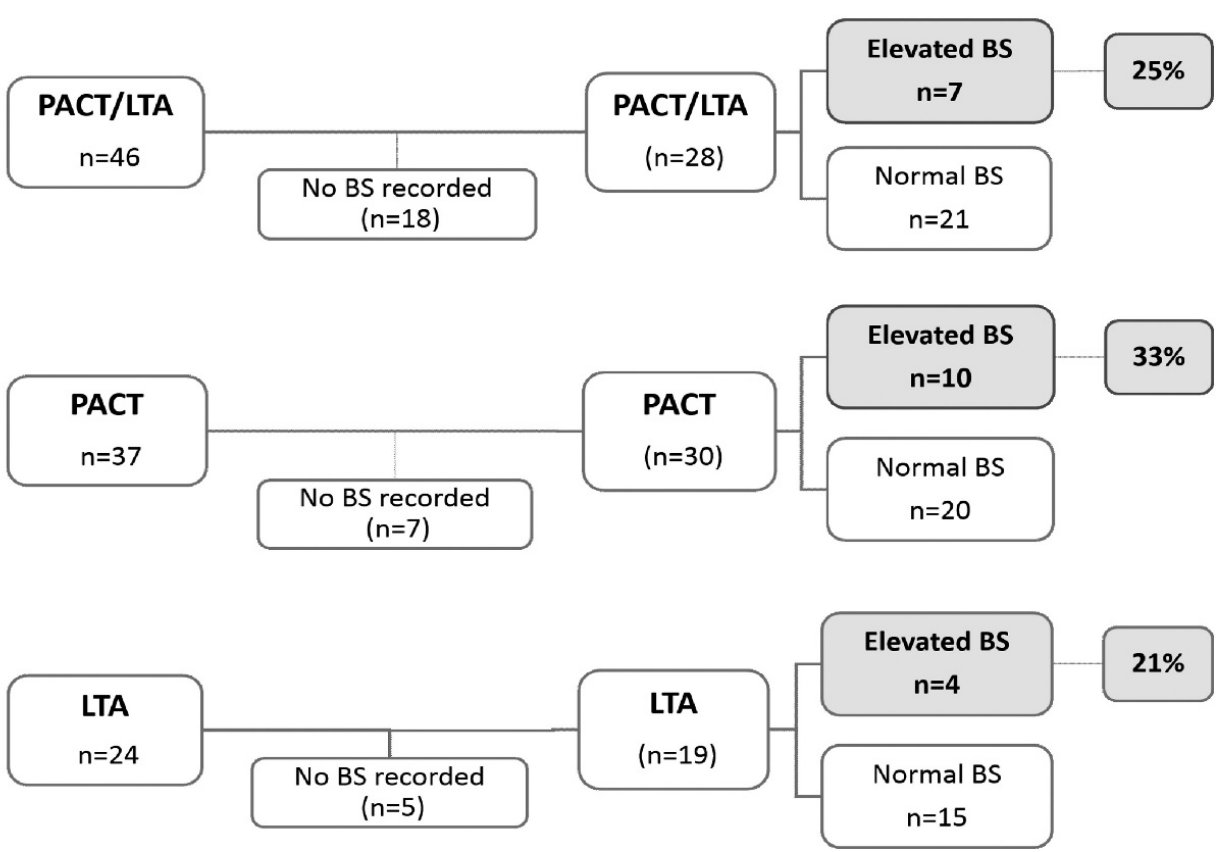

Figure 5. Agreement of bleeding score (BS) with PFD according to both LTA and WB-PACT (panel A), to only WB-PACT (panel B) and to only LTA (panel C).

\section{DISCUSSION}

This study investigates the (additional) value of flow cytometric analysis of platelet function in patients with suspected thrombocytopathy. 
As expected, we observed a weak correlation between the total scores of all parameters/ agonists of LTA and WB-PACT in the study population. It was in line with our expectations that there was no complete correlation, because there are major differences between LTA (a semi-quantitative method) and whole blood PACT (a quantitative analysis) [25]. Our findings and findings of others [19] show that LTA and WB-PACT are complimentary tests, which give supplemental insights in the bleeding risk, indicating that the tests improve each other's prediction.

Our data seem to demonstrate that LTA is insensitive to detect deviations in VWF concentration or function by measuring platelet agglutination induced by high concentrations of ristocetin as none of the patients had an abnormal response (score $>1$ ). Indeed, LTA is mostly used to detect gain-of-function defects VWD (type2B, platelet type) by low ristocetin induced agglutination [8]. Interestingly, WB-PACT was able to detect a reduced binding of VWF in response to ristocetin in a population of patients with suspected thrombocytopathy, and data corresponded well with decreased VWF:Ag and/or VWF:RCo. Other differences between the LTA and the WB-PACT include different agonists that were used (Table 2). Unlike LTA, the WB-PACT does not contain a thromboxane analogue, AA or epinephrine, however, it does include a PAR-1 activating peptide. In the past we validated U46619 and epinephrine with WB-PACT, however both agonists gave too much variation between measurements. The triple helical crosslinked CRP is a powerful GPVI agonist, however, CRP is sensitive to GPVI density on the platelet surface. In contrast, although GPVI plays a crucial role in collagen-dependent thrombus formation, it shows a low affinity for collagen and the adhesion of platelets on collagen is considerably enhanced by interaction with integrin a2 $\beta 1[26,27]$. Together, in response to collagen, $a 2 \beta 1$ and GPVI synergistically stimulate $\mathrm{Ca}^{2+}$ signaling, and platelet aggregation $[26,28]$. Furthermore, a limitation of this study is the determination of the cut-off values for WB-PACT. The reference population only consisted of 56 healthy individuals instead of the 120 recommended by the CSLI guidelines and the $10^{\text {th }}$ percentile was used as the cut-off value. Also, the score model was not previously validated in a group of severe defined PFD. However, in a previous study, we showed that the PACT assay discriminates Bernard-Soulier from non-Bernard-Soulier by the absence of binding of the anti-GPIb antibody, while Glanzmann thrombasthenia is discriminated from non-Glanzmann thrombasthenia by the absence of allbß3 antibody binding to platelets [19].

In the population patients presenting with suspicion of bleeding diathesis, we show a weak agreement between the two tests (concordant in $62 \%$ of the cases, $k=0.23$ ). In other studies, this agreement was higher (concordant in $65 \%$ of the cases, $\mathrm{k}=0.32$ ), which may be explained by the different agonist panels and the different patient populations (exclusion of children, pregnancy, von Willebrand disease and factor deficiency) [19]. In 
patient populations with a history of excessive bleeding (median BS=13), the agreement between the tests is much higher (concordant in $84 \%$ of cases, $k=0.67$ ) [29] then in populations with milder bleeding phenotypes.

The unified ISTH-BAT, has gained general acceptance, and the uniform reporting of reference ranges according to age and gender allows standardization of the results and comparison between studies $[20,30]$. We observed that $28 \%$ of patients with elevated BS were not diagnosed with WB-PACT nor LTA and that $64 \%$ of patients with normal BS were detected with PFD according to LTA and/or WB-PACT. This is in contrast with the high negative predictive value of the ISTH-BAT reported earlier [31, 32]. However, a systemic review does not support this finding and report that, as disease prevalence in the studies was low (because most patients do not have a MBD) there are many truenegative test results, but the lower sensitivity in these studies indicates that the few patients with a MBD still could be missed by the ISTH-BAT [33].

In conclusion, in this population consisting of patients suspected for thrombocytopathy, a weak correlation was found between WB-PACT test results and the results from the widely used LTA test. Although $28 \%$ of patients with high BS were not detected with WBPACT nor LTA, all children with elevated BS had either a positive LTA or a positive WB PACT test. A major advantage of WB-PACT is that the ristocetin induced VWF-binding to platelets can be measured in exactly the same setting as platelet activation. Ristocetin induced VWF-binding to platelets is a representative measurement for VWF:Ag and VWF:RCo positivity. Therefore, based on the present results in combination with the advantages compared to LTA (little amount of blood needed, less preprocessing steps and quantitative results), WB-PACT may have added value for the routine diagnostic work-up in patients who need to have platelet function tested.

\section{Funding}

L.L. was supported by the China Scholarship Council (CSC) via the State Scholarship Fund (File No. 201706230245).

\section{Declaration of Interest}

The authors report no declarations of interest. Some of the authors are employees of the research company Synapse BV (member of the Diagnostica Stago group).

\section{Acknowledgements}

We would like to thank Taissa Adams, Diëgo Arnoe, Julie De Leenheer, An De Saar, Michael Luypaert and Esha Wauters for their technical support. 


\section{REFERENCES}

1. Quiroga T, Mezzano D: Is my patient a bleeder? A diagnostic framework for mild bleeding disorders. Hematology American Society of Hematology Education Program 2012, 2012:466-474.

2. Michelson AD: Platelets 3rd ed edn. Netherlands: Amsterdam : Academic Press; 2013.

3. Gresele P, Harrison P, Bury L, Falcinelli E, Gachet C, Hayward CP, Kenny D, Mezzano D, Mumford AD, Nugent D et al: Diagnosis of suspected inherited platelet function disorders: results of a worldwide survey. Journal of thrombosis and haemostasis : JTH 2014, 12(9):1562-1569.

4. Schafer Al: Bleeding and thrombosis in the myeloproliferative disorders. Blood 1984, 64(1):1-12.

5. George JN, Caen JP, Nurden AT: Glanzmann's thrombasthenia: the spectrum of clinical disease. Blood 1990, 75(7):1383-1395.

6. George JN, Shattil SJ: The clinical importance of acquired abnormalities of platelet function. The New England journal of medicine 1991, 324(1):27-39.

7. Hayward CPM: How I investigate for bleeding disorders. International journal of laboratory hematology 2018, 40 Suppl 1:6-14.

8. Hayward CPM, Moffat KA, Brunet J, Carlino SA, Plumhoff E, Meijer P, Zehnder JL: Update on diagnostic testing for platelet function disorders: What is practical and useful? International journal of laboratory hematology 2019, 41 Suppl 1:26-32.

9. Dawood BB, Lowe GC, Lordkipanidze M, Bem D, Daly ME, Makris M, Mumford A, Wilde JT, Watson SP: Evaluation of participants with suspected heritable platelet function disorders including recommendation and validation of a streamlined agonist panel. Blood 2012, 120(25):5041-5049.

10. Quiroga T, Goycoolea M, Panes O, Aranda E, Martínez C, Belmont S, Muñoz B, Zúñiga P, Pereira J, Mezzano D: High prevalence of bleeders of unknown cause among patients with inherited mucocutaneous bleeding. A prospective study of 280 patients and 299 controls. Haematologica 2007, 92(3):357-365.

11. Femia EA, Scavone M, Lecchi A, Cattaneo M: Effect of platelet count on platelet aggregation measured with impedance aggregometry (Multiplate analyzer) and with light transmission aggregometry. Journal of thrombosis and haemostasis : JTH 2013, 11(12):2193-2196.

12. Paniccia R, Priora R, Liotta AA, Abbate R: Platelet function tests: a comparative review. Vasc Health Risk Manag 2015, 11:133-148.

13. Michelson AD: Platelet function testing in cardiovascular diseases. Circulation 2004, 110(19):e489493.

14. Christie D, Avari T, Carrington L, Cohen E, De Biase B, Harrison P, Kickler T, Kottke-Marchant K, Ledford-Kraemer M, Rand ML et al: Platelet function testing by aggregometry: approved guideline. Clinical and Laboratory Standards Institute 2008, 28:1-45.

15. Hayward CP, Moffat KA, Raby A, Israels S, Plumhoff E, Flynn G, Zehnder JL: Development of North American consensus guidelines for medical laboratories that perform and interpret platelet function testing using light transmission aggregometry. American journal of clinical pathology 2010, 134(6):955-963.

16. Cattaneo M, Cerletti C, Harrison P, Hayward CP, Kenny D, Nugent D, Nurden P, Rao AK, Schmaier AH, 
Watson SP et al: Recommendations for the Standardization of Light Transmission Aggregometry: A Consensus of the Working Party from the Platelet Physiology Subcommittee of SSC/ISTH. Journal of thrombosis and haemostasis : JTH 2013.

17. Shattil SJ, Cunningham M, Hoxie JA: Detection of activated platelets in whole blood using activationdependent monoclonal antibodies and flow cytometry. Blood 1987, 70(1):307-315.

18. Boknas N, Ramstrom S, Faxalv L, Lindahl TL: Flow cytometry-based platelet function testing is predictive of symptom burden in a cohort of bleeders. Platelets 2017:1-8.

19. van Asten I, Schutgens REG, Baaij M, Zandstra J, Roest M, Pasterkamp G, Huisman A, Korporaal SJA, Urbanus RT: Validation of flow cytometric analysis of platelet function in patients with a suspected platelet function defect. Journal of thrombosis and haemostasis : JTH 2018, 16(4):689-698.

20. Rodeghiero F, Tosetto A, Abshire T, Arnold DM, Coller B, James P, Neunert C, Lillicrap D, VWF ISj, Perinatal/Pediatric Hemostasis Subcommittees Working G: ISTH/SSC bleeding assessment tool: a standardized questionnaire and a proposal for a new bleeding score for inherited bleeding disorders. Journal of thrombosis and haemostasis: JTH 2010, 8(9):2063-2065.

21. Achison M, Joel C, Hargreaves PG, Sage SO, Barnes MJ, Farndale RW: Signals elicited from human platelets by synthetic, triple helical, collagen-like peptides. Blood coagulation \& fibrinolysis : an international journal in haemostasis and thrombosis 1996, 7(2):149-152.

22. Huskens D, Sang Y, Konings J, van der Vorm L, de Laat B, Kelchtermans H, Roest M: Standardization and reference ranges for whole blood platelet function measurements using a flow cytometric platelet activation test. PloS one 2018, 13(2):e0192079.

23. van der Vorm LN, Li L, Huskens D, Chayouâ W, Kelchtermans H, de Groot PG, Roest M, Remijn JA, de Laat B: Analytical characterization and reference interval of an enzyme-linked immunosorbent assay for active von Willebrand factor. PloS one 2019, 14(2):e0211961.

24. Verfaillie CJ, De Witte E, Devreese KM: Validation of a new panel of automated chemiluminescence assays for von Willebrand factor antigen and activity in the screening for von Willebrand disease. International journal of laboratory hematology 2013, 35(5):555-565.

25. van Asten I, Schutgens REG, Urbanus RT: Toward Flow Cytometry Based Platelet Function Diagnostics. Seminars in thrombosis and hemostasis 2018, 44(3):197-205.

26. Nieswandt B, Watson SP: Platelet-collagen interaction: is GPVI the central receptor? Blood 2003, 102(2):449-461.

27. Moroi M, Jung SM: Platelet glycoprotein VI: its structure and function. Thromb Res 2004, 114(4):221233.

28. Kuijpers MJE, Schulte V, Bergmeier W, Lindhout T, Brakebusch C, Offermanns S, Fassler R, Heemskerk JWM, Nieswandt B: Complementary roles of platelet glycoprotein VI and integrin alpha 2 beta 1 in collagen-induced thrombus formation in flowing whole blood ex vivo. Faseb J 2003, 17(2):685-+.

29. Dovlatova N, Lordkipanidze M, Lowe GC, Dawood B, May J, Heptinstall S, Watson SP, Fox SC: Evaluation of a whole blood remote platelet function test for the diagnosis of mild bleeding disorders. Journal of thrombosis and haemostasis: JTH 2014, 12(5):660-665.

30. Elbatarny M, Mollah S, Grabell J, Bae S, Deforest M, Tuttle A, Hopman W, Clark DS, Mauer AC, Bowman M et al: Normal range of bleeding scores for the ISTH-BAT: adult and pediatric data from the merging 
project. Haemophilia : the official journal of the World Federation of Hemophilia 2014, 20(6):831-835.

31. Rashid A, Moiz B, Karim F, Shaikh MS, Mansoori H, Raheem A: Use of ISTH bleeding assessment tool to predict inherited platelet dysfunction in resource constrained settings. Scandinavian journal of clinical and laboratory investigation 2016, 76(5):373-378.

32. Adler M, Kaufmann J, Alberio L, Nagler M: Diagnostic utility of the ISTH bleeding assessment tool in patients with suspected platelet function disorders. Journal of thrombosis and haemostasis : JTH 2019.

33. Moenen F, Nelemans PJ, Schols SEM, Schouten HC, Henskens YMC, Beckers EAM: The diagnostic accuracy of bleeding assessment tools for the identification of patients with mild bleeding disorders: A systematic review. Haemophilia: the official journal of the World Federation of Hemophilia 2018, 24(4):525-535. 


\section{SUPPLEMENTAL FIGURES}

A

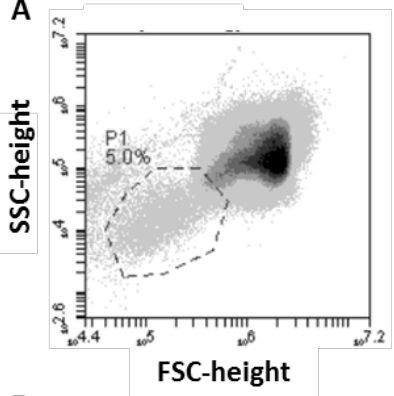

D

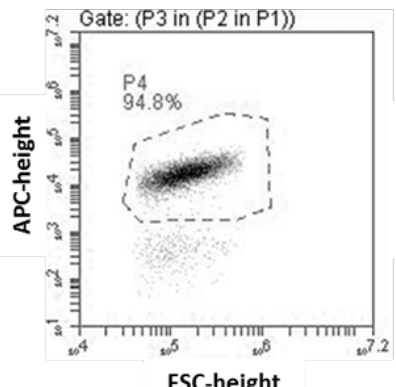

B

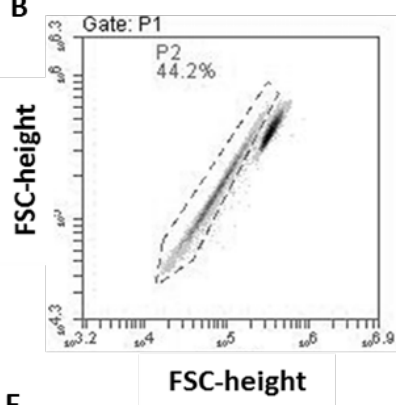

$\mathrm{E}$

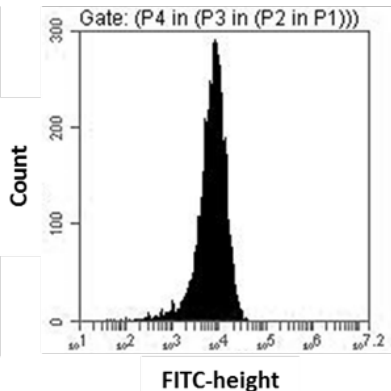

C

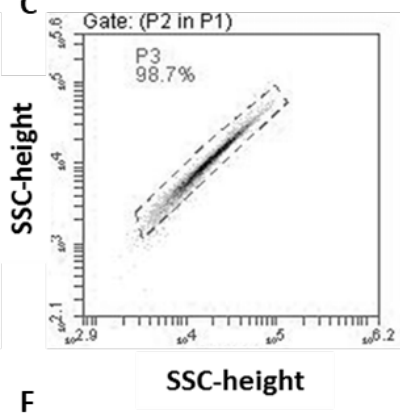

$\mathrm{F}$

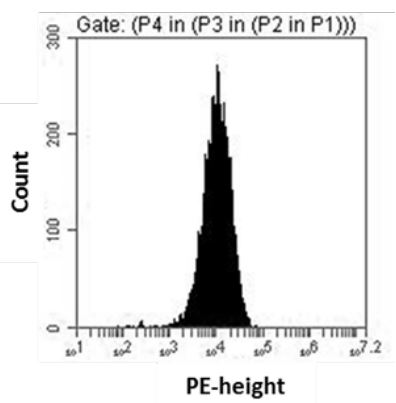

Supplemental figure 1. Gating strategy WB-PACT. In a dot plot of FSC-height versus SSC-height, a gate P1 is created around the platelets (panel A). To discriminate doublets, FSC-height versus FSC-area gated on region "P1" was plotted and a gate P2 was placed around the singlets (panel B). Subsequently, SSC-height versus SSC-area gated on region "P2 in P1" was plotted and gate P3 was placed around the singlets (panel C). Subsequently, a FSC-height versus APC-height plot gated on region "P3 in (P2 in P1)" (panel D). Platelets were stained with anti-GPIb antibody conjugated with APC. In this plot the platelets will appear as APC positive and a gate P4 is placed around these platelets. Finally, histograms FITC-height (panel E) and PE-height (panel F) were created gated

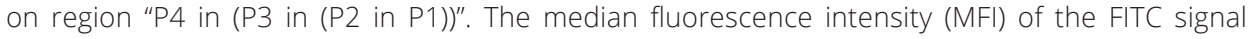
corresponds to allb $\beta 3$ receptor activation or VWF binding and the MFI of the PE signal corresponds with the P-selectin expression. 

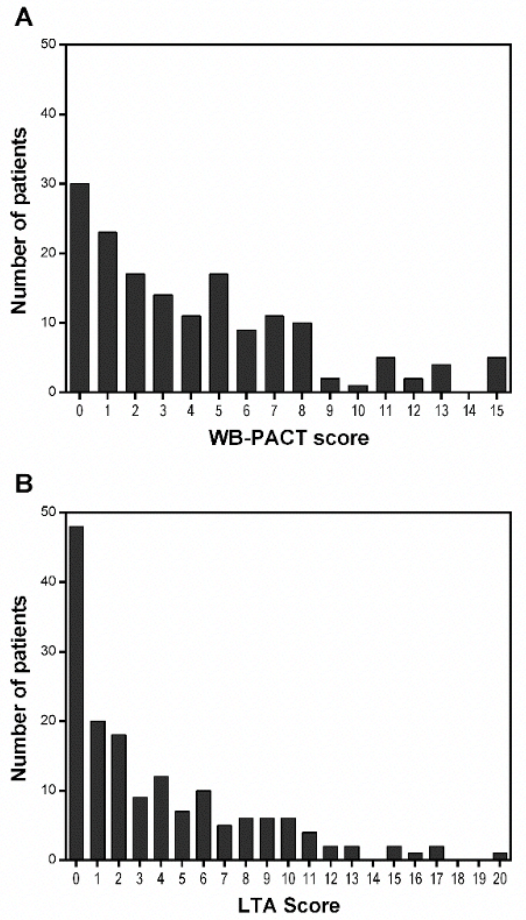

Supplemental figure 2. Distribution of total scores among patients with bleeding diathesis according to WB-PACT (panel A) and LTA (panel B). 
A

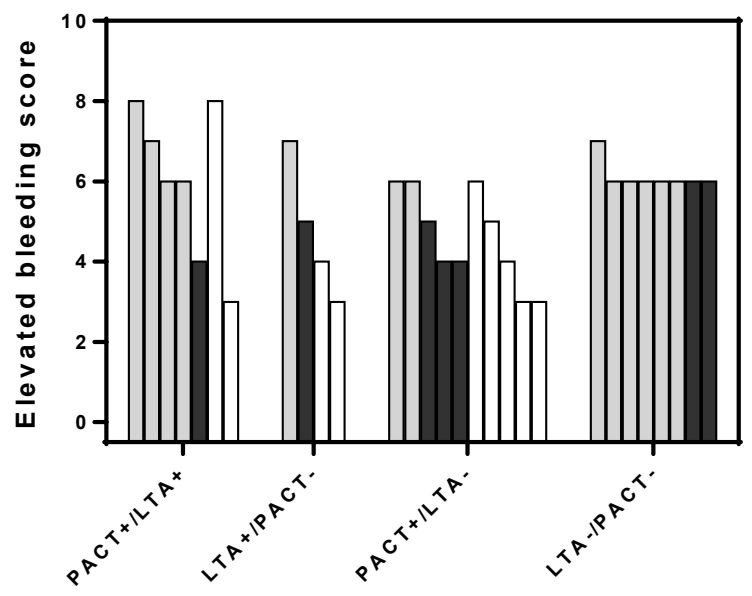

B

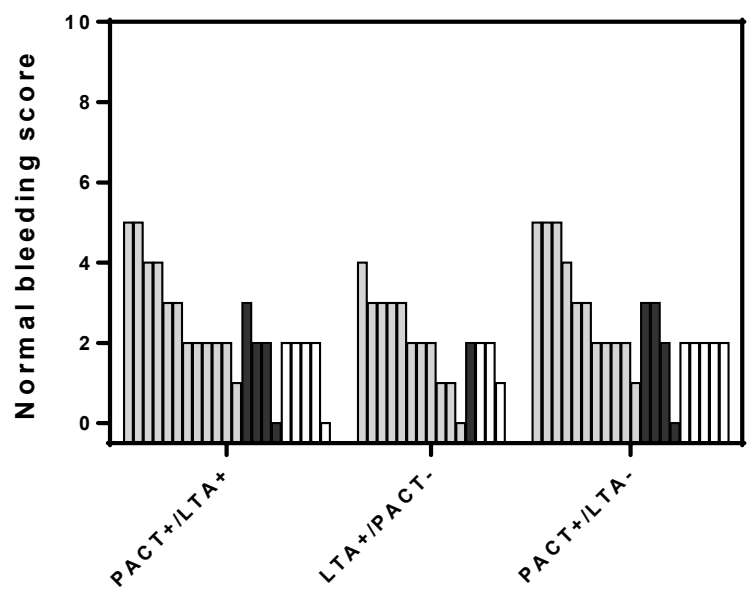

C

\section{LTA+/PACT+ LTA+/PACT- LTA-/PACT+ LTA-/PACT-}

\begin{tabular}{|c|c|c|c|c|}
\hline \multicolumn{5}{|l|}{ Patients with elevated BS } \\
\hline number of patients (29) & $7(24 \%)$ & $4(14 \%)$ & $10(34 \%)$ & $8(28 \%)$ \\
\hline \multicolumn{5}{|l|}{ Patients with low BS } \\
\hline number of patients (88) & $21(24 \%)$ & 15 (17\%) & $20(23 \%)$ & $32(36 \%)$ \\
\hline
\end{tabular}

Supplemental figure 3. Occurrence of test results among individual women (grey bars), men (dark grey bars) and children (white bars) with elevated bleeding score (panel A) and normal bleeding score (panel B). Table showing occurrence of test results among patients with elevated bleeding score and normal bleeding score (panel C). 



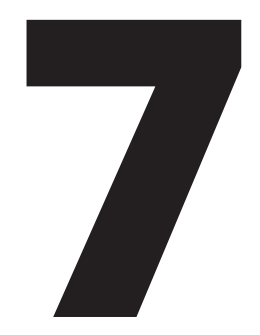

\section{Analytical characterization and reference interval of an enzyme-linked immunosorbent assay for active von Willebrand factor}

Lisa N. van der Vorm

$\mathrm{Li} \mathrm{Li}$

Dana Huskens

Walid Chayoua

Hilde Kelchtermans

Philip G. de Groot

Mark Roest

Jasper A. Remijn

Bas de Laat 


\section{ABSTRACT}

\section{Background}

Interaction of von Willebrand factor (VWF) with platelets requires a conformational change that exposes an epitope within the VWF A1 domain, enabling platelet glycoprotein Iba binding. Quantification of this "active" conformation of VWF has been shown to provide pathophysiological insight into conditions characterized by excessive VWF-platelet interaction.

\section{Methods}

We developed an immunosorbent assay based on a variable heavy chain antibody fragment against the VWF A1 domain as a capture antibody. Assay performance in terms of specificity (binding to active R1306W- and sheared VWF), precision, accuracy, linearity, limits of detection and stability were determined. Active VWF, VWF antigen, VWF ristocetin cofactor activity, VWF:GP1bM and VWF propeptide were measured in citrated plasma and platelet- VWF binding in whole blood from 120 healthy individuals to establish a reference interval for active VWF and to assess associations with other VWF parameters.

\section{Results}

Intra- and inter-assay CVs were between 2.4-7.2\% and 4.1-9.4\%, depending on the level. Mean recovery of spiked recombinant R1306W VWF was $103 \pm 3 \%$. The assay was linear in the range of $90.1-424.5 \%$ and had a limit of quantification of $101 \%$. The reference interval for active VWF was $91.6-154.8 \%$ of NPP. Significant, positive correlations between active VWF and all other VWF parameters were found, with the strongest correlation with VWF: GP1bM binding.

\section{Conclusions}

We developed and validated an immunosorbent assay for the accurate detection of active VWF levels in plasma. The assay fulfilled all analytical criteria in this study and a reference interval was established, allowing its use to quantify active VWF in pathological conditions for future research. 


\section{INTRODUCTION}

Von Willebrand factor (VWF) is a multimeric plasma protein that mediates platelet adhesion and platelet-platelet interactions [1]. VWF binds via its A3 domain to exposed subendothelial collagen at sites of vascular injury. Collagen-bound VWF tethers platelets to the vessel wall via transient interaction of its A1 domain with the platelet glycoprotein (GP)Ib-IX-V receptor complex [2]. Circulating VWF can only exert this function after conversion from its latent, globular conformation to an active conformation, in which the binding site for platelet Gplba is exposed. Under physiological conditions, conversion to this active state is well regulated.

Upon vascular injury, VWF immobilization to subendothelial collagen in conjunction with increased shear stress induce VWF unfolding [3], allowing for platelet-VWF interaction [4].

Various pathological conditions are associated with premature and/or excessive formation of VWF-platelet aggregates [5]. Von Willebrand disease (VWD) type 2B, for instance, is characterized by increased interactions between VWF and platelets, resulting from gain-of-function mutations (e.g. R1306W) in the VWF A1 domain [6]. Consequently, these patients lack high-molecular-weight VWF multimers and suffer from thrombocytopenia, clinically resulting in a bleeding phenotype [7]. In thrombotic thrombocytopenic purpura (TTP) patients, an acquired or inherited deficiency of the VWF cleaving protease ADAMTS13 results in accumulation of ultra large (UL)-VWF multimers [8]. Clinically the result is a thrombotic phenotype caused by platelet-rich thrombi occluding the microvasculature [8]. Spontaneous VWF-plate- let interaction in these conditions is indicative of the presence of VWF in its active conformation.

Several laboratory tests to assess VWF are available. The VWF antigen (VWF:Ag) assay is a quantitative assay that provides an overall level of VWF present in plasma, but yields no information concerning the quality of VWF in terms of its ability to bind platelets [9]. The ristocetin cofactor (VWF:RCo) assay is the most widely used method to assess the functional activity of VWF [10]. However, VWF:RCo assays are highly variable [11] and the need for ristocetin to activate VWF does not reflect the in vivo situation. A more recently developed assay uses recombinant GPIb fragments with two gain-of-function mutations that allow binding to VWF in the absence of ristocetin (VWF:GPIbM) [12]. Although this VWF:GPIbM assay eliminates the need for the non-physiological activator ristocetin, it is based on non-physiological binding of VWF to a mutant receptor. Therefore, an immunosorbent assay to directly detect circulat- ing VWF in its active conformation was previously developed [13]. This assay is based on a recombinant llama-derived antibody (AUNWFa-11) that preferentially binds the Gplba- binding conformation of the VWF A1 domain [13]. Using this assay, elevated active VWF levels were identified in plasma from 
patients with VWD type 2B [13], TTP [13], HELLP syn- drome [14], systemic inflammatory response syndrome [15], antiphospholipid syndrome [16], diabetes [17], first ST-segment elevation myocardial infarction (STEMI) [18], sickle cell disease [19], malaria [20] and dengue [21]. Together, these findings have provided new insights into the presence of, as well as the mechanism behind increased active VWF in these pathological conditions. Moreover, quantification of active VWF has potential value for diagnostics, for example to differentiate VWD 2B from other types of VWD, to distinguish between forms of VWD 2B and to differentiate between patients with acquired and congenital TTP [13, 22].

However, there are no reports of an analytical validation of this assay. Therefore, we developed our own in-house immunosorbent assay to measure active VWF, also based on a llama- derived variable heavy chain antibody fragment $(\mathrm{VHH})$ directed against a cryptic epitope in the A1 domain of VWF. The aims of the current study were to (1) characterize the analytical performance of this assay; (2) provide a reference interval for active VWF and (3) determine correlations between this assay and other, established VWF assays and a whole blood flow cytometric assay for platelet-VWF binding (Plt:VWF binding, adapted from others [23-25]).

\section{MATERIALS AND METHODS}

\section{Ethics statement}

The research complied with all the relevant national regulations, institutional policies and the tenets of the Helsinki Declaration, and has been evaluated by the Medical Ethical Committee (METC) of Maastricht University Medical Center/University of Maastricht (METC reference 152015). Participants gave full written informed consent.

\section{Reagents}

Haemate P (CSL Behring, Pennsylvania, USA) VWF/FVIII concentrate (stock 12 mg/mL) was used as native human VWF in its globular conformation, referred to as "HVWF". Recombinant VWF with the VWD type 2B R1306W (stock $17 \mu \mathrm{g} / \mathrm{mL}$ ) or R1306Q (stock 55 $\mu \mathrm{g} / \mathrm{mL}$ ) mutation (produced as described previously [26]) was used as VWF constitutively in its active conformation. Polyclonal rabbit anti-VWF antibody conjugated to HRP was purchased from Dako (P0226, Glostrup, Denmark). Bovine serum albumin (BSA; A7906), SIGMAFAST O- Phenylenediamine dihydrochloride (OPD; P9187) and sulfuric acid (H2SO4; 339741) were all purchased from Sigma-Aldrich (Zwijndrecht, The Netherlands).

\section{Production of $\mathrm{VHH}$}

See S1 Methods for a detailed description of VHH production. 


\section{Study population and plasma preparation}

The study population consisted of 120 healthy individuals, aged 18-65 years, recruited between March 12, 2018 and May 17, 2018. Participants did not take any anti-coagulant or anti-platelet drugs for at least one week and did not have a history of thrombosis or bleeding. Analytical characterization of the assay was performed using residual, anonymized citrated plasma from the reference value study samples as well as residual, anonymized citrated plasma initially collected for routine laboratory diagnostics conducted at the Gelre Hospitals Apeldoorn, the Netherlands. Blood was collected by antecubital venipuncture into vacuum tubes (1 volume trisodium citrate $0.105 \mathrm{M}$ to 9 volumes blood) (BD Vacutainer System, Becton Dickinson, Franklin Lakes, USA). Cell counts in whole blood were performed with a Coulter Counter analyzer (Beckman Coulter, Woerden, The Netherlands). Plasma was prepared by centrifugation at 2,840 g for 10 minutes, pipetting off the plasma fraction, and repeating centrifugation to obtain the platelet-poor plasma fraction, which was stored at $-80^{\circ} \mathrm{C}$.

\section{Active VWF immunosorbent assay}

The active VWF assay is based on a llama-derived single-domain antibody $(\mathrm{VHH})$ directed against a cryptic epitope in the A1 domain of VWF (Fig 1), which is only exposed upon unfolding of VWF [13]. Briefly, 96 wells microtiter plates (NUNC Maxisorp, Thermo Fisher Scientific, Waltham, USA) were coated overnight at $4^{\circ} \mathrm{C}$ with $1.98 \mu \mathrm{g} / \mathrm{ml} \mathrm{VHH}$ against active VWF in a carbonate-bicarbonate coating buffer ( $\mathrm{pH}$ 9.6), followed by a blocking step with 2\% BSA in phosphate-buffered saline (PBS) for 45 minutes at room temperature (RT). After washing with 0.01\% Tween-20 in PBS, plasma samples (diluted 1:20 in PBS/1\% BSA) were incubated for 2 hours at RT. Following another wash-step, the wells were incubated with HRP- conjugated anti-VWF antibodies $(1.2 \mu \mathrm{g} / \mathrm{mL})$ in PBS/1\% BSA for 2 hours at room temperature. Plates were then washed again before addition of SIGMAFAST OPD (Sigma). The reaction was stopped with $3 \mathrm{M}$ sulfuric acid (H2SO4, Sigma). Optical densities (OD) were measured at 490 nm using an ELx808 Absorbance Microplate Reader (Biotek, Bad Friedrich- shall, Germany). Results were normalized (\%) to normal pooled plasma (NPP) on the same plate. This differs from the quantification method in earlier studies using the previous version of this assay, reporting the VWF activation factor [13]. The VWF activation factor was calculated by dividing the absorbance slope of the dilution curves for a patient sample by the slope of a NPP sample. We simplified the assay and reduced required sample volume by measuring one dilution of each plasma sample and expressing its active VWF level compared to that in NPP. For the NPP, blood from 10 healthy control donors was collected. After double centrifugation (2,840 g for $10 \mathrm{~min}$ ), plasmas were pooled and aliquots of $1 \mathrm{ml}$ were stored at $-80^{\circ} \mathrm{C}$ until use in the active VWF ELISA. Of note, NPP contains only a very small amount of active VWF. If an individual has an active VWF level of 150\%, he/she has 1.5 times more circulating active VWF than is present in the NPP, which is still a minute amount, but the current assay is sensitive enough to detect this difference. 


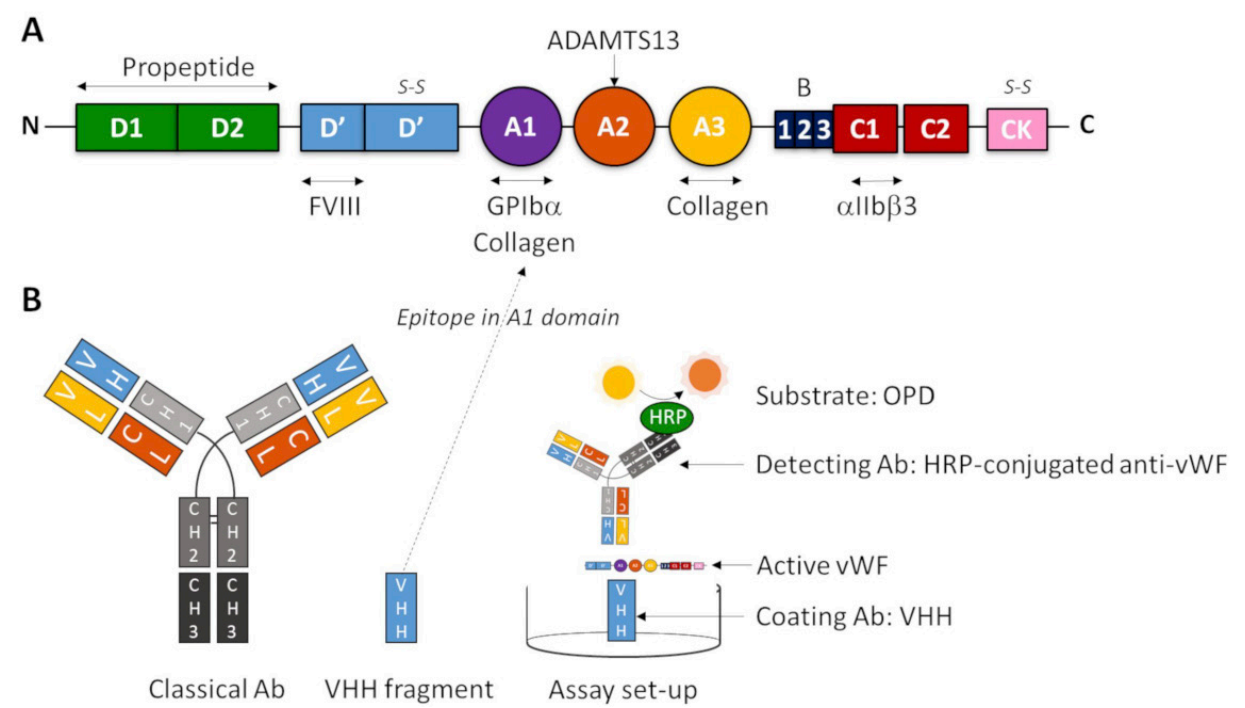

Figure 1. Scheme for measurement of active VWF. (A) The protein domain structure of mature VWF (adapted from Crawley et al.[27]). (B) The set-up of the immunosorbent assay described here, based on a variable heavy chain antibody fragment $(\mathrm{VHH})$ directed against a cryptic epitope in the A1 domain of VWF as capture antibody, and a polyclonal rabbit anti-human VWF antibody conjugated with HRP as detecting antibody.

\section{Assay performance studies}

See S1 Methods for a detailed description of the assay performance studies.

\section{Established VWF parameters}

VWF antigen (VWF:Ag) levels were determined using the Liatest VWF assay on an automated STA-R Max coagulation analyser (Diagnostica Stago, Leiden, the Netherlands). VWF ristocetin cofactor activity (VWF:RCo) was measured with the automated chemiluminescent HemosIL VWF:RCo assay (Werfen-Instrumentation Laboratory, Bedford, USA). VWF propeptide (VWFpp) was measured using the anti-human VWFpp MW1939 antibody pair and Tool Set 2 (Sanquin, Amsterdam, The Netherlands) [28] according to the manufacturer instructions, with the exception that VWFpp was expressed as a \% of the level in NPP included the same plate (due to limited number of available kits the recommended 8 step 2-fold serial dilution curve could not be included) VWF:GP1bM was measured using the INNOVANCE VWF Ac assay on a Sysmex CS-2500 instrument (Siemens Healthcare Diagnostics GmbH, Marburg, Germany) according to the manufacturer's protocol.

\section{Flow cytometric analysis of PIt:VWF binding}

See S1 Methods for a detailed description of this assay, for which standardization and a 
reference range were described previously [29].

\section{Statistical analysis}

Outlier analysis was performed using Tukey's hinges. One outlier in the active VWF values was detected (167.9\%) but there was no evidence of an experimental error and this value was hence not excluded. Normality was tested using Q-Q plots and the Shapiro-Wilk test. None of the VWF parameters were normally distributed. Continuous variables were expressed as median and interquartile range (25\%-75\%). Reference intervals were obtained using nonparametric calculation, according to the latest CLSI guidelines [30], i.e. the 2.5th percentile to the 97.5th percentile of the distribution. Associations between variables were determined using Spearman's rank correlation coefficient. Groups were compared using the Mann-Whitney $U$ test for independent samples (continuous variables) or Chi-square test for categorical variables. Multiple linear regression was used to adjust for the effect of differences in VWF:Ag levels on associations between (1) blood group and VWF:RCo, VWF:GP1bM and Plt:VWF binding, and (2) sex and active VWF levels. A p-value of 0.05 was considered statistically significant for all comparisons. Analyses were performed using Statistical Package for Social Sci- ences (SPSS Incorporate, Chicago, USA) version 25. Figures were prepared using GraphPad Prism version 5.00 (Graphpad Software, San Diego, USA).

\section{RESULTS}

\section{VHH specifically detects active VWF}

To test the specificity of the $\mathrm{VHH}$ for the active conformation of $\mathrm{VWF}$, its binding to HVWF, R1306W VWF, R1306Q VWF, and VWF in NPP was investigated (Fig 2A). The VHH neither interacted with HVWF nor VWF in NPP, as apparent from the weak (background) signal at $490 \mathrm{~nm}$. In contrast, binding of the $\mathrm{VHH}$ to constitutively active R1306W and R1306Q VWF resulted in a strong dose-dependent signal. To confirm that the VHH also recognizes native VWF that is activated by high shear stress we also tested vortexed NPP. As a result, the VHH interacted with the shear-unfolded VWF in NPP. Thus, the VHH specifically recognizes VWF in its active conformation.

\section{Analytical performance of active VWF assay}

We proceeded with the analytical characterization of the assay, summarized in Table 1 and depicted in Fig 2B-2F. Precision studies revealed intra-assay CVs of 2.4-7.2\% and inter-assay CVs of 4.1-9.4\%, depending on measurand level (Fig 2B). Spiked R1306W VWF could be accurately measured in a plasma matrix at concentrations as low as 0.25 $\mu \mathrm{g} / \mathrm{mL}$, with observed levels well within $90-110 \%$ of the expected levels (Fig 2C). The assay was linear (R2>0.99) in almost the entire range of dilutions of plasma spiked with 
R1306W (Fig 2D and Table 1).
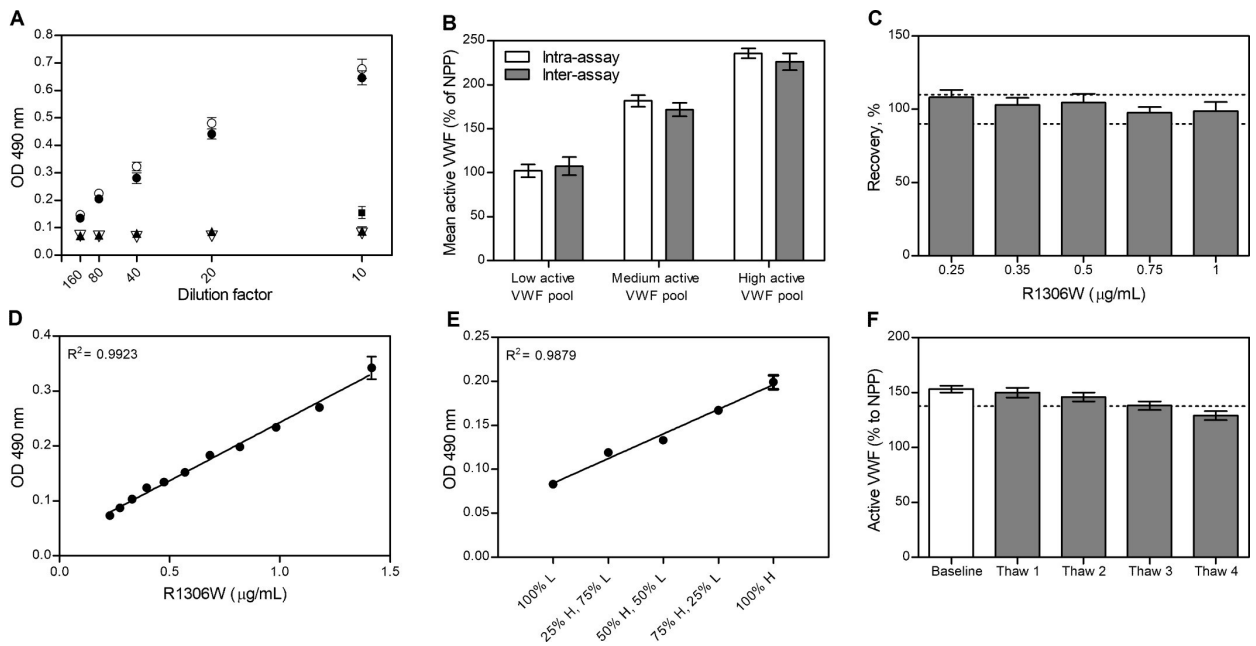

Figure 2. Analytical characteristics of the active VWF assay. (A) Binding of the VHH to R1306W $\operatorname{VWF}(\cdot)$, R1306Q VWF ( ), HVWF ( $)$ ), VWF in NPP under static conditions $(\boldsymbol{\Delta})$ and NPP after vortexing to simulate shear stress $(\cdot$, only $10 x$ diluted). Dilution factors are indicated at the $x$-axis. (B) Mean active VWF levels in plasma pools (with low (91-110\%), medium (150-200\%) and high (>220\%) active VWF levels) with corresponding SDs for 20 replicates on a single plate on the same day (intra-assay, white bars) or on 20 separate days (inter-assay, grey bars). (C) Recovery (\%) of increasing levels of R1306W VWF spiked to plasma pool. The acceptance range of 90-110\% of expected values is indicated by dotted lines. (D) Assay response (OD at $490 \mathrm{~nm}$ ) for a 12-step, 1.2-fold dilution series of plasma spiked with R1306W. Linear regression parameters ( \pm SD) are: slope 0,21 \pm 0.006 , intercept $0.03 \pm 0.005$, SD of residuals 0.007. (E) Low (L) and high $(H)$ active VWF plasma pools were mixed in varying proportions to assess dilutional linearity. (F) Stability of active VWF in a plasma pool at baseline and after multiple freeze-thaw steps (Thaw 1-4). The acceptance criterium of $<10 \%$ analyte loss is indicated by the dotted line. Data represent the mean $\pm S D(n=3)$ unless otherwise stated.

Table 1. Analytical performance characteristics of the active VWF ELISA assay.

\begin{tabular}{|c|c|c|}
\hline Parameter & Assay performance & \\
\hline Precision & $\begin{array}{l}\text { Intra-assay (Mean [SD;\%CV]) } \\
\text { L 102\% [7.3\%; 7.2\%] } \\
\text { M 182\% [6.6\%; 3.7\%] } \\
\text { H 235\% [5.7\%; } 2.4 \%]\end{array}$ & $\begin{array}{l}\text { Inter-assay (Mean [SD;\%CV]) } \\
\text { L 107\% [10.1\%; 9.4\%] } \\
\text { M 172\% [7.6\%; 4.4\%] } \\
\text { H 226\% [9.2\%; 4.1\%] }\end{array}$ \\
\hline Accuracy & 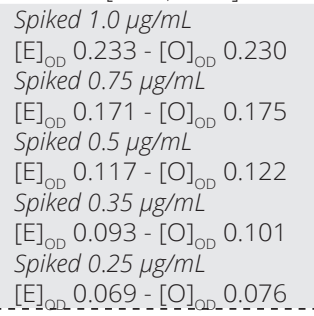 & 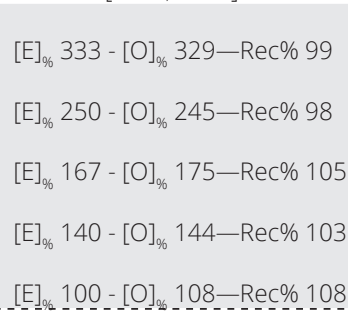 \\
\hline
\end{tabular}


Table 1. Continued

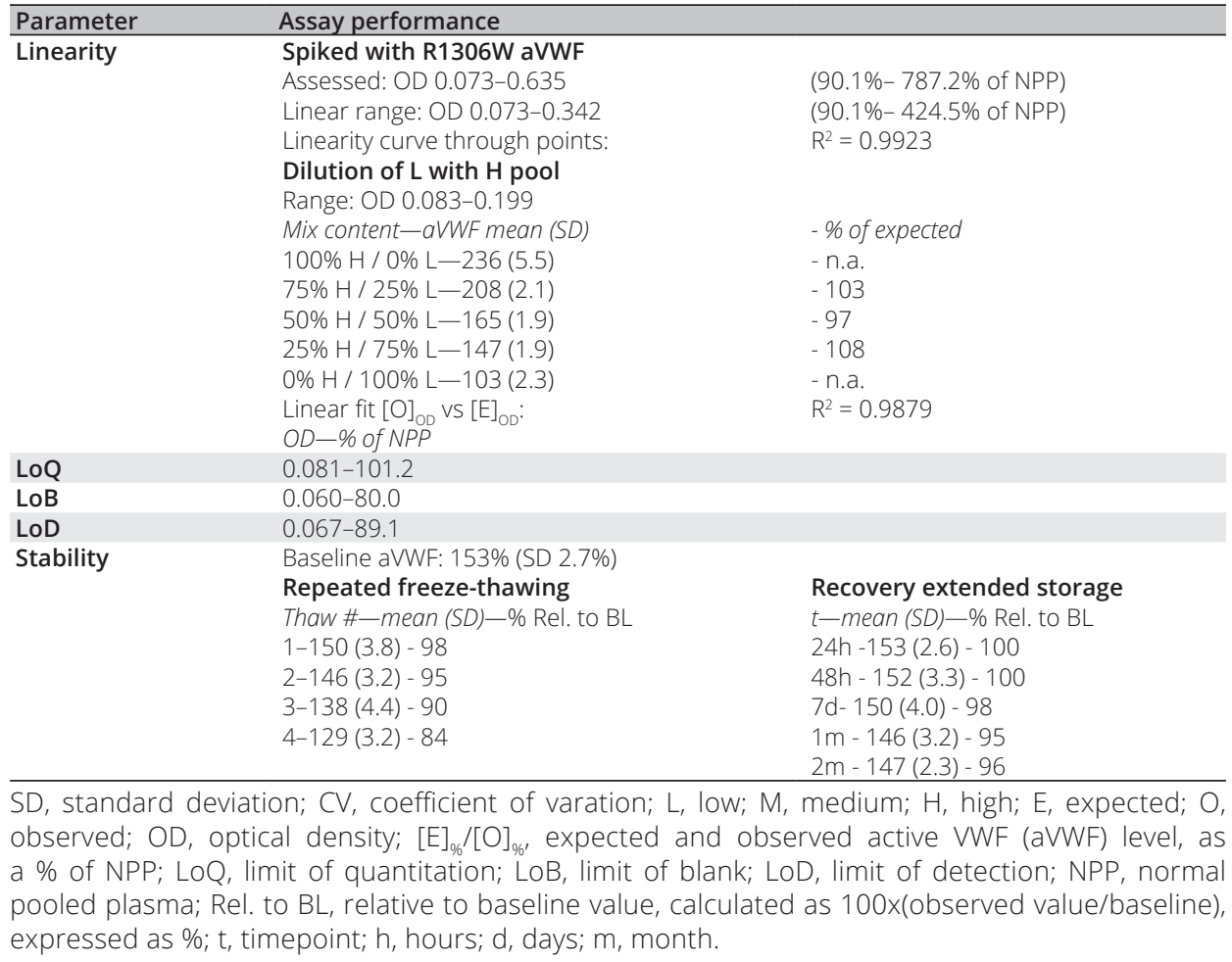

Recovery of active VWF in mixes of low $(L)$ and high $(H)$ plasma pools was within the predefined acceptable limits of 90-110\%. Moreover, the curve fitted through the 5 points for these mixes (expected OD based on levels in individual pools versus observed) was linear (R2 = 0.99) (Fig 2E). The limit of quantitation (LOQ) was 101\% (corresponding to an OD of 0.081 ). The limit of blank (LOB) and limit of detection (LOD) were 80.0\% (OD 0.060) and $89.1 \%$ (OD 0.067), respectively. Active VWF in citrated plasma was stable for at least 2 months (longest time tested) at $-80^{\circ} \mathrm{C}$ (Table 1). Importantly, freeze-thawing up to 3 times slightly decreased the detected active VWF, whereas more frequent freezethawing resulted in loss of analyte exceeding the criterion of 10\% (Fig 2F).

\section{Reference interval and inter-individual variation for (active) VWF}

Demographic data and blood counts of 120 healthy individuals are summarized in Table 2. Hemoglobin levels, hematocrit and red blood cell counts were significantly higher in male compared to female subjects. The distribution of active VWF (S1 Fig) in this population showed a clear right-skewedness, with the majority of values approximating that in NPP (100\%). The corresponding reference interval for active VWF was 91.6154.8\%. Reference intervals for VWF:Ag, VWF:RCo, VWF: GP1bM, VWFpp and Plt:VWF binding were 65.1-189.9\%, 47.0-161.5\%, 58.3-189.7\%, 73.3-205\% and $1.4-15.6 \%$ 
respectively (Table 3). Moreover, active VWF had a considerably lower inter-individual variation (15.1\%) than all other VWF parameters.

Table 2. Demographic and laboratory results of healthy donors in reference interval study.

\begin{tabular}{|c|c|c|c|c|}
\hline Parameter & All & Male $(n=60)$ & Female $(n=60)$ & p-value \\
\hline \multicolumn{5}{|l|}{ General } \\
\hline Age (years) & $31(25-44.5)$ & $29(25-39)$ & $32.5(26-50)$ & ns \\
\hline OC use (\%) & 15 & - & $15(25 \%)$ & - \\
\hline \multicolumn{5}{|l|}{ Haematological } \\
\hline \multirow{4}{*}{ Blood group $(\%)$} & $O \mathrm{n}=26(40 \%)$ & $n=12(41 \%)$ & $n=14(39 \%)$ & ns \\
\hline & $A n=29(45 \%)$ & $n=12(41 \%)$ & $n=17(47 \%)$ & \\
\hline & $B n=9(14 \%)$ & $\mathrm{n}=5(17 \%)$ & $n=4(11 \%)$ & \\
\hline & $A B n=1(1 \%)$ & & $n=1(3 \%)$ & \\
\hline Platelets (x109/L) & $233(208-270)$ & 229 (207-257) & $244(216-284)$ & ns \\
\hline WBC $\left(\times 10^{9} / L\right)$ & $5.2(4.6-6.0)$ & $5.2(4.6-5.8)$ & $5.3(4.7-6.2)$ & ns \\
\hline $\mathrm{RBC}\left(\mathrm{x} 10^{12} / \mathrm{L}\right)$ & $4.5(4.2-4.7)$ & $4.6(4.5-4.8)$ & $4.3(4.1-4.5)$ & $<0.0001$ \\
\hline $\mathrm{Hb}(\mathrm{mmol} / \mathrm{L})$ & $7.9(7.5-8.4)$ & $8.3(8.0-8.6)$ & $7.6(7.2-7.9)$ & $<0.0001$ \\
\hline $\mathrm{Hct}(\mathrm{L} / \mathrm{L})$ & $0.39(0.37-0.41)$ & $0.40(0.39-0.41)$ & $0.37(0.35-0.39)$ & $<0.0001$ \\
\hline MPV (fL) & $7.5(7.1-8.2)$ & $7.4(7.1-8.2)$ & $7.6(7.2-8.3)$ & ns \\
\hline
\end{tabular}

OC, oral contraceptives; freq, frequency; WBC, white blood cell count; RBC, red blood cell count; $\mathrm{Hb}$, haemoglobin; Hct, haematocrit; MPV, mean platelet volume. ${ }^{a}$ Blood group known for 65 individuals (36 female, 29 male), percentages indicate proportions for each blood group from total individuals with known blood group. Medians and interquartile ranges (25-75\%) are given unless otherwise indicated.

\section{Mutual correlations of VWF parameters}

A variety of assays may be performed for the assessment of VWF in plasma, each quantifying a different form of VWF (globular native VWF:Ag, active VWF, VWFpp) or providing qualitative information on its function (VWF:RCo, VWF:GP1bM, Plt:VWF binding). Significant correlations were observed between all these VWF parameters in our study population. Active VWF levels were significantly and positively correlated with VWFpp ( $r=0.281, p=0.005)$, Plt:VWF binding (0.273, $p=0.003)$, VWF:Ag levels $(r=$ $0.390, p<0.001), V W F: R C o(r=0.401, p<0.001)$ and the highest correlation was found with VWF:GP1bM ( $r=0.464, p<0.001)$ (Fig 3 and S1 Table). Overall, the highest degree of correlation ( $p<0.001$ ) existed between VWF:GP1 bM activity and VWF-platelet binding as measured by the Plt:VWF binding assay. There were also strong associations between these assays and the other two functional assays for active VWF and VWF:RCo, and between the functional assays and VWF:Ag (S1 Table). 
Table 3. Reference intervals for active VWF and other VWF parameters in 120 healthy individuals.

\begin{tabular}{lllllllll}
\hline Parameter & Mean & SD & \%CV & Median & IQR & Ref. interval 2.5\%-97.5\% & Min & Max \\
\hline VWF:Act (\%) & 115.4 & 17.4 & 15.1 & 112.9 & 25.1 & $91.6-154.8$ & 91.0 & 167.9 \\
VWF:Ag (\%) & 115.1 & 32.2 & 28.0 & 104.5 & 39.8 & $65.1-189.9$ & 40.0 & 218.0 \\
VWF:RCo (\%) & 103.9 & 32.9 & 31.7 & 102.6 & 48.1 & $47.0-161.5$ & 20.8 & 243.4 \\
VWF:GP1bM (\%) & 112.9 & 32.9 & 29.1 & 110.6 & 43.1 & $58.3-189.7$ & 41.3 & 229.5 \\
VWFpp (\%) & 121.8 & 23.1 & 24.1 & 121.2 & 32.1 & $73.3-188.7$ & 60.3 & 210.3 \\
Plt:VWF (\%) & 6.9 & 3.2 & 46.4 & 6.4 & 3.8 & $1.4-15.6$ & 0.9 & 18.0 \\
\hline
\end{tabular}

$\% C V$, inter-individual variation expressed as coefficient of variation, calculated as (SD/Mean)*100; IQR, interquartile range, calculated as $75^{\text {th }}-25^{\text {th }}$ percentile; Ref. interval, reference interval, calculated as the $2.5^{\text {th }}$ percentile to the $97.5^{\text {th }}$ percentile of the distribution; VWF:Act, active VWF, \% of level in NPP. VWF:Ag, VWF antigen; VWF:RCo, VWF ristocetin cofactor activity; VWF:GP1 bM, VWF binding to gain-of-function GP1b fragments; VWFpp, VWF propeptide, \% of level in NPP; Plt:VWF, platelet-VWF binding, \% of signal for beads.
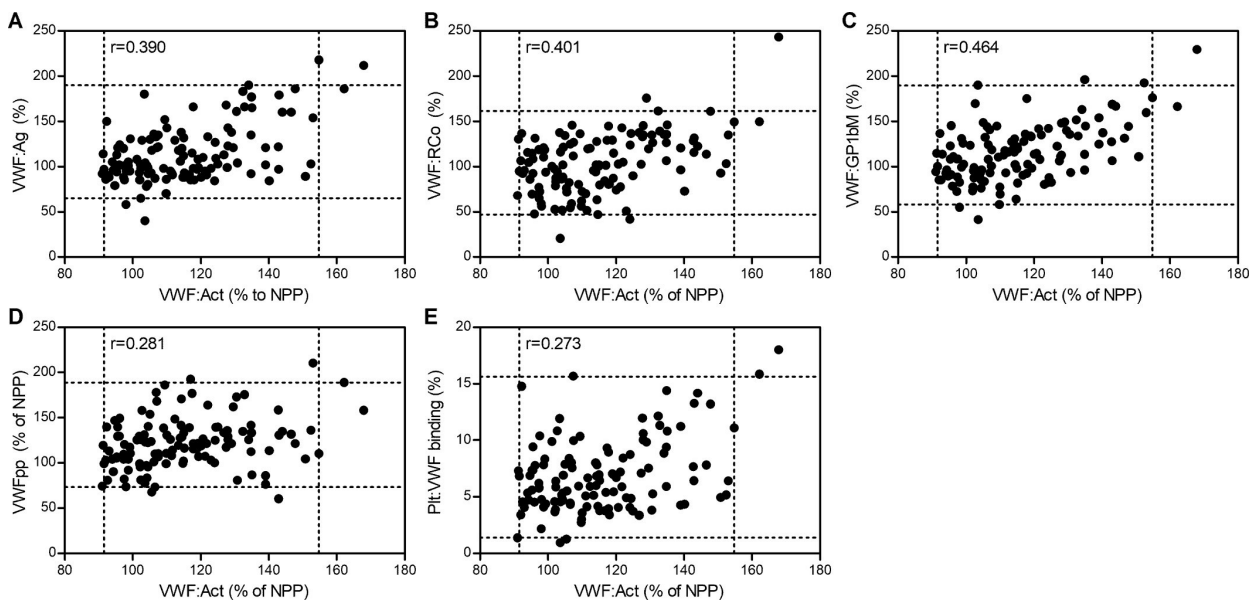

Figure 3. Correlation of active VWF with other VWF parameters. Scatterplots and corresponding Spearman rank correlations ( $r$ ) between active VWF assay (VWF:Act) and (A) VWF:Ag, (B) VWF:RCo, (C) VWF:GP1bM, (D) VWFpp and (E) Plt:VWF binding. Dotted lines delineate reference intervals determined in this study, with the centre square containing 95\% of values. Spearman rank correlation coefficients $(r)$ are indicated in the upper left corner of each panel.

\section{VWF parameters related to clinical parameters}

Active VWF levels correlated with gender $(r=0.21, p=0.019)$ and were significantly $(p=$ 0.033 ) higher in male (median 115.2\%, IQR 105.6-127.4\%) compared to female subjects (107.1\%, IQR 97.5-126.1\%). Therefore, sex-specific reference intervals were calculated, but these did not differ considerably (93.3-158.5\% for men, 91.2-159.7\% for women), and corresponding confidence intervals overlapped (S2 Fig). VWF:Ag levels were also significantly ( $p=0.044$ ) higher in male (median 112.5\%, IQR 95.5-135.3\%) compared to female subjects (median 99.5\%, IQR 91.0-122.5\%). Adjustment for VWF:Ag levels using multiple linear regression strongly reduced the association between active VWF and sex $(p=0.047$ to $p=0.289)$. Active VWF did not correlate with age, whereas significant positive correlations of VWF:Ag levels $(r=0.236, p=0.009)$ and VWF:RCo $(r=0.181, p=$ 
0.048) with age were observed (S3 Fig). No association between active VWF levels and blood group was found ( $r=0.196, p=0.118)$, while VWF:Ag levels $(r=0.296, p=0.018)$, VWF:RCo ( $r=0.299, p=0.017), V W F: G P 1 B m(r=0.280, p=0.025)$ and Plt:VWF binding $(r=0.293, p=0.021)$ were significantly lower in $O$ compared to non-O blood group subjects (S4 Fig). However, in multiple linear regression analysis adjustment for VWF:Ag levels eliminated the significant association between VWF:RCo ( $p=0.270)$, VWF:GP1bM $(p=0.297)$ and Plt:VWF binding $(p=0.612)$ with blood group.

\section{DISCUSSION}

Here, we describe an immunosorbent assay for the reliable, accurate and specific quantification of active VWF in citrated plasma. We established its reference interval in a healthy population and we demonstrated a clear association of active VWF levels with four clinically established VWF assays and a flow cytometric VWF-platelet binding assay. The active VWF assay fulfilled our pre-defined analytical requirements. First, the assay was highly specific for active VWF, as only the two recombinant VWF proteins that are constitutively in their active conformation were detected and no cross-reactivity with native (NPP) and purified HVWF was observed. This is in accordance with findings by Hulstein et al [13]. Of note, serial dilutions of NPP did not substantially increase the OD since NPP only contains minute amounts of active VWF. Reproducibility was adequate, with values below $10 \%$ for both the intra- and inter-assay CV, comparable with those found by others $[13,18]$. All values obtained from the population of healthy individuals were above the LoD (89.1\%), whereas 26 (22\%) values were below the LoQ of $101 \%$. This is not necessarily a limitation, since high active VWF levels are much more relevant as they may induce thrombosis or bleeding. Lastly, active VWF showed good stability in samples stored at $-80^{\circ} \mathrm{C}$, and a maximum of 3 freeze-thawing steps should be allowed to prevent analyte loss of more than $10 \%$. This effect of freeze-thawing may be explained by an increased proportion of small molecular weight fragments, as was observed previously [31]. Taken together, these data demonstrate the robust analytical performance of this assay.

In order to identify elevated active VWF levels in patient samples, it is essential to establish a reference interval in healthy individuals. To this end, we determined active VWF levels in 120 healthy individuals, as recommended by CLSI guidelines [30]. The reference range shows a right-skewed distribution, with most individuals having very Iow active VWF levels, similar to that in NPP (set to 100\%). The right skew indicates that slightly more active VWF is present in native plasma of some healthy individuals, and these small differences could be detected due to the high sensitivity of our assay. The reference interval includes the majority of active VWF levels reported by others, although 
values below the LoD of the current assay were previously reported [16, 20, 21, 32]. This difference may be attributed to an alternative calculation method for the VWF'activation factor' (see limitations below) [13]. Of note, the number of healthy controls in previous studies was much lower than 120, ranging from nine [13, 14] to fifty-nine [17].

In addition, we determined VWF:Ag, VWF:RCo, VWF:GP1 bM, VWFpp and Plt:VWF binding in these 120 samples. Increased active VWF can potentially have major impact on the risk of bleeding and/or thrombosis, as exemplified by VWD type 2B and TTP [13]. Therefore, regulatory mechanisms, for instance the natural inhibitor $\beta 2 \mathrm{GPI}$, are keeping active VWF levels low in healthy individuals [16]. The inter-individual variation in active VWF levels (15.1\%) was nearly half of that in VWF:Ag levels and the majority of active VWF values were close to the level in NPP (100\%). The observed inter-individual variation for VWF:Ag was similar to the previously reported inter-individual biologic variation for VWF [33].

We identified significant positive correlations between all VWF parameters (S1 Table). With regard to active VWF, the highest correlation was found with two other activity assays, namely VWF:RCo and VWF:GP1bM as expected based on assay principles. Active VWF circulates in a GPIba-binding conformation and will therefore contribute to the chemiluminescent signal in the VWF:RCo assay, generated when VWF (either active VWF already present or VWF activated by ristocetin) binds to microparticles coated with GPIba. Similarly, in the VWF:GP1 bM assay only active VWF can bind spontaneously to the microparticle-(mutant) GP1b complex, inducing agglutination that is measured turbidimetrically. Whereas the VWF: GP1 bM and VWF:RCo assays are much more widely established, advantages of the active VWF ELISA assay are that neither non-physiological additives (ristocetin or mutant GP1b) nor specialized instruments are required. Moreover, active VWF correlated well with VWF:Ag levels ( $r=0.390)$, showing that with increasing VWF the amount of circulating VWF in its active, unfolded conformation was also higher. This is in accordance with correlations found between VWF activation factor and VWF:Ag in previous studies in patients with a first STEMI ( $r=0.58)$ [18] and malignant hypertension ( $r=0.62$ [34]. The stronger correlation in these studies may be explained by reporting the activation factor, which corrects for differences in VWF:Ag levels. Moreover, active VWF correlated with VWFpp. Relatively increased VWFpp is commonly used as a marker for acute endothelial cell activation, while relatively high VWF: Ag levels are considered a marker for more chronic endothelial cell activation [28]. VWF in endothelial cells is likely in its active conformation, as freshly secreted VWF is able to interact with the platelet Gplb-IX-V complex [5]. Thus, it can be postulated that circulating active VWF was secreted from endothelial cells (hence correlating with VWFpp and VWF:Ag levels), but escaped ADAMTS-13 proteolysis.

In our healthy population, active VWF and VWF:Ag levels were significantly higher in men 
than in women In contrast to the activation factor calculation method [13], our calculation of active VWF levels does not correct for differences in VWF:Ag levels. Therefore, sexrelated differences in active VWF levels have not been reported in previous studies. Whereas the major- ity of the literature on the influence of sex (gender) on VWF:Ag reports no differences [9, 35], Conlan et al. reported higher VWF:Ag levels in female compared to male subjects [36], while Campos et al. found higher VWF:Ag levels in men than in women [37]. In addition, both older age and non-O blood group were associated with higher VWF:Ag levels, as described in literature [9, 38-41]. VWF:RCo, VWF:GP1bM and PIt:VWF binding were also higher in indi- viduals with a non-O blood group, but correction for VWF:Ag levels rendered these associa- tions non-significant.

A possible limitation of the current assay is that it expresses the amount of active VWF as a percentage, normalized to values in NPP. We consider this a more reliable approach than expressing the absolute concentration with regard to a monomeric VWF A1 domain, because one VHH binds active VWF multimers with different sizes [42] and hence variable numbers of VWF epitopes. The assay cannot adjust for variation in the multimeric composition between individuals. In addition, in order to calculate the concentration of active VWF, we would need to assume that the $\mathrm{VHH}$ recognizes mutation-induced activated VWF (e.g. R1306W) similarly as non-mutated (activated) VWF and as active VWF in a variety of conditions. Because we are uncertain of these assumptions, we propose that expressing active VWF as a percentage of NPP is a more reliable calibrator for the moment. However, the normalized result makes it more difficult to standardize the assay, and the reported reference interval may have been slightly different using another batch of NPP. Given the small inter-individual varia- tion in active VWF in healthy individuals and the large increases observed in several pathologies [5], we do not expect minor differences in NPP active VWF levels to cause wrong classification of active VWF in a sample as pathological.

\section{CONCLUSIONS}

Previously, it has been shown that several pathological conditions are associated with strongly increased active VWF levels [5]. The underlying mechanism can likely be attributed to (a com- bination of) three causes: (1) changes in the (conformation of) VWF itself (e.g. VWD type 2B [13]), (2) changes in endothelial secretion (e.g. malaria [20], diabetes [17]), processing or clearance of VWF (e.g. TTP [13], HELLP [14]) or (3) increased shear stress (e.g. mild aortic stenosis [32], first STEMI [18]). Increased circulating active VWF levels may also be present in other conditions associated with infection and/or inflammation, for instance in chronic kidney dis- ease (submitted). Moreover, using the current assay, we found that active VWF levels in healthy individuals increase following 
strenuous exercise [43]. Quantification of active VWF in a variety of diseases could provide valuable pathophysiological insight for diagnosis and development of new treatment strategies. The current validation of an immunosorbent assay for active VWF and the establishment of a reference interval will facilitate future studies investigating the role of active VWF in health and disease.

\section{Acknowledgments}

We would like to thank V. Verschuur, G. Oelemans-Prins, W. Gerritsen, M. KoldenhofVisser, I. Bakker-Wolbers and B. Wildeboer (Gelre Hospitals Apeldoorn) for assistance with the VWF:Ag and VWF:RCo measurements and M. van Wijnen, M. Siderius and B. Blankenspoor (Meander Medical Center Amersfoort) for their help with the VWF:GP1 bM measurements. We are grateful to our colleagues at Synapse Research Institute Maastricht for recruiting donors and for blood drawing and processing.

\section{Author Contributions}

Conceptualization: Lisa N. van der Vorm, Dana Huskens, Hilde Kelchtermans, Philip G. de Groot, Mark Roest, Jasper A. Remijn, Bas de Laat.

Data curation: Lisa N. van der Vorm, Dana Huskens.

Formal analysis: Lisa N. van der Vorm, Li Li, Dana Huskens.

Investigation: Lisa N. van der Vorm, Li Li, Dana Huskens, Walid Chayoua^, Hilde Kelchtermans.

Methodology: Lisa N. van der Vorm, Dana Huskens, Walid Chayoua`, Hilde Kelchtermans, Philip G. de Groot, Mark Roest, Jasper A. Remijn, Bas de Laat.

Project administration: Lisa N. van der Vorm, Li Li, Dana Huskens, Mark Roest.

Resources: Jasper A. Remijn, Bas de Laat.

Supervision: Dana Huskens, Hilde Kelchtermans, Philip G. de Groot, Mark Roest, Jasper A. Remijn, Bas de Laat.

Validation: Lisa N. van der Vorm.

Visualization: Lisa N. van der Vorm.

Writing - original draft: Lisa N. van der Vorm.

Writing - review \& editing: Lisa N. van der Vorm, Li Li, Dana Huskens, Walid Chayoua^, Hilde Kelchtermans, Philip G. de Groot, Mark Roest, Jasper A. Remijn, Bas de Laat. 


\section{REFERENCES}

1. Lenting PJ, Christophe OD, Denis CV. von Willebrand factor biosynthesis, secretion, and clearance: connecting the far ends. Blood. 2015; 125(13):2019-28. https://doi.org/10.1182/ blood-2014-06-528406 PMID: 25712991

2. Savage B, Saldivar E, Ruggeri ZM. Initiation of platelet adhesion by arrest onto fibrinogen or translocation on von Willebrand factor. Cell. 1996; 84(2):289-97. PMID: 8565074

3. Springer TA. von Willebrand factor, Jedi knight of the bloodstream. Blood. 2014; 124(9):1412-25. https://doi.org/10.1182/blood-2014-05-378638 PMID: 24928861

4. Huizinga EG, Tsuji S, Romijn RA, Schiphorst ME, de Groot PG, Sixma JJ, et al. Structures of glycoprotein Ibalpha and its complex with von Willebrand factor A1 domain. Science. 2002; 297(5584):11769. https://doi.org/10.1126/science.107355 PMID: 12183630

5. Groot E, de Groot PG, Fijnheer R, Lenting PJ. The presence of active von Willebrand factor under various pathological conditions. Curr Opin Hematol. 2007; 14(3):284-9. https://doi.org/10.1097/MOH. Ob013e3280dce531 PMID: 17414220

6. Ruggeri ZM, Zimmerman TS. von Willebrand factor and von Willebrand disease. Blood. 1987; 70 (4):895-904. PMID: 3307951

7. Sharma R, Flood VH. Advances in the diagnosis and treatment of Von Willebrand disease. Hematology Am Soc Hematol Educ Program. 2017; 2017(1):379-84. https://doi.org/10.1182/ asheducation-2017.1. 379 PMID: 29222282

8. Sadler JE. Von Willebrand factor, ADAMTS13, and thrombotic thrombocytopenic purpura. Blood. 2008; 112(1):11-8. https://doi.org/10.1182/blood-2008-02-078170 PMID: 18574040

9. Favaloro EJ, Soltani S, McDonald J, Grezchnik E, Easton L, Favaloro JW. Reassessment of ABO blood group, sex, and age on laboratory parameters used to diagnose von Willebrand disorder: potential influ- ence on the diagnosis vs the potential association with risk of thrombosis. Am J Clin Pathol. 2005; 124 (6):910-7. PMID: 16416741

10. Lippi G, Franchini M, Salvagno GL, Montagnana M, Poli G, Guidi GC. Correlation between von Willebrand factor antigen, von Willebrand factor ristocetin cofactor activity and factor VIII activity in plasma. J Thromb Thrombolysis. 2008; 26(2):150-3. https://doi.org/10.1007/s11239-007-0090-0 PMID: 17786534

11. Patzke J, Favaloro EJ. Laboratory Testing for von Willebrand Factor Activity by Glycoprotein Ib Binding Assays (VWF:GPIb). Methods Mol Biol. 2017; 1646:453-60. https://doi.org/10.1007/978-1-49397196-1_33 PMID: 28804847

12. Patzke J, Budde U, Huber A, Mendez A, Muth H, Obser T, et al. Performance evaluation and multicentre study of a von Willebrand factor activity assay based on GPIb binding in the absence of ristocetin. Blood Coagul Fibrinolysis. 2014; 25(8):860-70. https://doi.org/10.1097/MBC.0000000000000169 PMID: 25192242

13. Hulstein JJ, de Groot PG, Silence K, Veyradier A, Fijnheer R, Lenting PJ. A novel nanobody that detects the gain-of-function phenotype of von Willebrand factor in ADAMTS13 deficiency and von Willebrand disease type 2B. Blood. 2005; 106(9):3035-42. https://doi.org/10.1182/blood-2005-03-1153 PMID: 
16014562

14. Hulstein JJ, van Runnard Heimel PJ, Franx A, Lenting PJ, Bruinse HW, Silence K, et al. Acute activation of the endothelium results in increased levels of active von Willebrand factor in hemolysis, elevated liver enzymes and low platelets (HELLP) syndrome. J Thromb Haemost. 2006; 4(12):2569-75. https:// doi. org/10.1111/j.1538-7836.2006.02205.x PMID: 16968329

15. Hyseni A, Kemperman H, de Lange DW, Kesecioglu J, de Groot PG, Roest M. Active von Willebrand factor predicts 28-day mortality in patients with systemic inflammatory response syndrome. Blood. 2014; 123(14):2153-6. https://doi.org/10.1182/blood-2013-08-508093 PMID: 24458436

16. Hulstein JJ, Lenting PJ, de Laat B, Derksen RH, Fijnheer R, de Groot PG. beta2-Glycoprotein I inhibits von Willebrand factor dependent platelet adhesion and aggregation. Blood. 2007; 110(5):1483-91. https://doi.org/10.1182/blood-2006-10-053199 PMID: 17488878

17. Chen SF, Xia ZL, Han JJ, Wang YT, Wang JY, Pan SD, et al. Increased active von Willebrand factor during disease development in the aging diabetic patient population. Age (Dordr). 2013; 35(1):171-7.

18. Rutten B, Maseri A, Cianflone D, Laricchia A, Cristell NA, Durante A, et al. Plasma levels of active Von Willebrand factor are increased in patients with first ST-segment elevation myocardial infarction: a multi- center and multiethnic study. Eur Heart J Acute Cardiovasc Care. 2015; 4(1):64-74. https://doi. org/10. 1177/2048872614534388 PMID: 24833640

19. Chen J, Hobbs WE, Le J, Lenting PJ, de Groot PG, Lopez JA. The rate of hemolysis in sickle cell disease correlates with the quantity of active von Willebrand factor in the plasma. Blood. 2011; 117 (13):3680-3. https://doi.org/10.1182/blood-2010-08-302539 PMID: 21300978

20. de Mast Q, Groot E, Lenting PJ, de Groot PG, McCall M, Sauerwein RW, et al. Thrombocytopenia and release of activated von Willebrand Factor during early Plasmodium falciparum malaria. J Infect Dis. 2007; 196(4):622-8. https://doi.org/10.1086/519844 PMID: 17624850

21. Djamiatun K, van der Ven AJ, de Groot PG, Faradz SM, Hapsari D, Dolmans WM, et al. Severe dengue is associated with consumption of von Willebrand factor and its cleaving enzyme ADAMTS-13. PLoS Negl Trop Dis. 2012; 6(5):e1628. https://doi.org/10.1371/journal.pntd.0001628 PMID: 22563509

22. Casonato A, Pontara E, Morpurgo M, Sartorello F, De Groot PG, Cattini MG, et al. Higher and lower active circulating VWF levels: different facets of von Willebrand disease. Br J Haematol. 2015; 171 (5):845-53. https://doi.org/10.1111/bjh.13785 PMID: 26456374

23. van Asten I, Schutgens REG, Baaij M, Zandstra J, Roest M, Pasterkamp G, et al. Validation of flow cytometric analysis of platelet function in patients with a suspected platelet function defect. J Thromb Hae- most. 2018; 16(4):689-98. https://doi.org/10.1111/jth.13952 PMID: 29337406

24. Giannini S, Cecchetti L, Mezzasoma AM, Gresele P. Diagnosis of platelet-type von Willebrand disease by flow cytometry. Haematologica. 2010; 95(6):1021-4. https://doi.org/10.3324/haematol.2009.015990 PMID: 19951970

25. Giannini S, Mezzasoma AM, Leone M, Gresele P. Laboratory diagnosis and monitoring of desmopressin treatment of von Willebrand's disease by flow cytometry. Haematologica. 2007; 92(12):1647-54. https://doi.org/10.3324/haematol.11313 PMID: 18055988

26. Lankhof H, Damas C, Schiphorst ME, MJ IJ, Bracke M, Sixma JJ, et al. Functional studies on platelet adhesion with recombinant von Willebrand factor type 2B mutants R543Q and R543W under 
conditions of flow. Blood. 1997; 89(8):2766-72. PMID: 9108394

27. Crawley JT, de Groot R, Xiang Y, Luken BM, Lane DA. Unraveling the scissile bond: how ADAMTS13 recognizes and cleaves von Willebrand factor. Blood. 2011; 118(12):3212-21. https://doi. org/10.1182/ blood-2011-02-306597 PMID: 21715306

28. van Mourik JA, Boertjes R, Huisveld IA, Fijnvandraat K, Pajkrt D, van Genderen PJ, et al. von Willebrand factor propeptide in vascular disorders: A tool to distinguish between acute and chronic endothelial cell perturbation. Blood. 1999; 94(1):179-85. PMID: 10381511

29. Huskens D, Sang Y, Konings J, van der Vorm L, de Laat B, Kelchtermans H, et al. Standardization and reference ranges for whole blood platelet function measurements using a flow cytometric platelet activa- tion test. PLoS One. 2018; 13(2):e0192079. https://doi.org/10.1371/journal.pone.0192079 PMID: 29389990

30. CLSI and IFCC-Ad. Defining, establishing and verifying reference intervals in the clinical laboratory: approved guideline-third edition. 2008. p. 1-76.

31. Zimmerman TS, Dent JA, Ruggeri ZM, Nannini LH. Subunit composition of plasma von Willebrand fac- tor. Cleavage is present in normal individuals, increased in IIA and IIB von Willebrand disease, but mini- mal in variants with aberrant structure of individual oligomers (types IIC, IID, and IIE). J Clin Invest. 1986; 77(3):947-51. https://doi.org/10.1172/JCI112394 PMID: 3485111

32. Hollestelle MJ, Loots CM, Squizzato A, Renne T, Bouma BJ, de Groot PG, et al. Decreased active von Willebrand factor level owing to shear stress in aortic stenosis patients. J Thromb Haemost. 2011; 9 (5):953-8. https://doi.org/10.1111/j.1538-7836.2011.04247.x PMID: 21352469

33. Ricos C, Alvarez V, Cava F, Garcia-Lario JV, Hernandez A, Jimenez CV, et al. Desirable specifications for total error, imprecision, and bias, derived from intra- and inter-individual biologic variation. [updated 2014; cited 20186 July]. Available from: http://www.westgard.com/biodatabase1.htm.

34. van den Born BJ, van der Hoeven NV, Groot E, Lenting PJ, Meijers JC, Levi M, et al. Association between thrombotic microangiopathy and reduced ADAMTS13 activity in malignant hypertension. Hypertension. 2008; 51(4):862-6. https://doi.org/10.1161/HYPERTENSIONAHA.107.103127 PMID: 18332284

35. Zhou Z, Yu F, Buchanan A, Fu Y, Campos M, Wu KK, et al. Possible race and gender divergence in association of genetic variations with plasma von Willebrand factor: a study of ARIC and 1000 genome cohorts. PLoS One. 2014; 9(1):e84810. https://doi.org/10.1371/journal.pone.0084810 PMID: 24465435

36. Conlan MG, Folsom AR, Finch A, Davis CE, Sorlie P, Marcucci G, et al. Associations of factor VIII and von Willebrand factor with age, race, sex, and risk factors for atherosclerosis. The Atherosclerosis Risk in Communities (ARIC) Study. Thromb Haemost. 1993; 70(3):380-5. PMID: 8259533

37. Campos M, Sun W, Yu F, Barbalic M, Tang W, Chambless LE, et al. Genetic determinants of plasma von Willebrand factor antigen levels: a target gene SNP and haplotype analysis of ARIC cohort. Blood. 2011; 117(19):5224-30. https://doi.org/10.1182/blood-2010-08-300152 PMID: 21343614

38. Gallinaro L, Cattini MG, Sztukowska M, Padrini R, Sartorello F, Pontara E, et al. A shorter von Willebrand factor survival in O blood group subjects explains how ABO determinants influence plasma von Willebrand factor. Blood. 2008; 111(7):3540-5. https://doi.org/10.1182/blood-2007-11-122945 
PMID: 18245665

39. Rydz N, Grabell J, Lillicrap D, James PD. Changes in von Willebrand factor level and von Willebrand activity with age in type 1 von Willebrand disease. Haemophilia. 2015; 21(5):636-41. https://doi. org/10. 1111/hae.12664 PMID: 25756206

40. Moeller A, Weippert-Kretschmer M, Prinz H, Kretschmer V. Influence of ABO blood groups on primary hemostasis. Transfusion. 2001; 41(1):56-60. PMID: 11161246

41. Albanez S, Ogiwara K, Michels A, Hopman W, Grabell J, James P, et al. Aging and ABO blood type influence von Willebrand factor and factor VIII levels through interrelated mechanisms. J Thromb Hae- most. 2016; 14(5):953-63. https://doi.org/10.1111/jth.13294 PMID: 26875505

42. Groot E, Fijnheer R, Sebastian SA, de Groot PG, Lenting PJ. The active conformation of von Willebrand factor in patients with thrombotic thrombocytopenic purpura in remission. J Thromb Haemost. 2009; 7 (6):962-9. https://doi.org/10.1111/j.1538-7836.2009.03433.x PMID: 19548905

43. van der Vorm LN, Huskens D, Kicken CH, Remijn JA, Roest M, de Laat B, et al. Effects of Repeated Bouts of Exercise on the Hemostatic System. Semin Thromb Hemost. 2018; 44(8):710-22. https:// doi. org/10.1055/s-0038-1673619 PMID: 30290394 


\section{SUPPLEMENTAL INFORMATION}

\section{S1 Methods}

\section{Production of $\mathrm{VHH}$}

VWF A1 domain specific VHH antibodies (MW 16.4 kDa) were commercially produced by U-Protein Express BV (Utrecht, the Netherlands). Briefly, HEK293E-253 cells were transfected with endotoxin-free maxiprep DNA for the desired sequence of the antiVWF VHH (based on the sequence provided by ${ }^{1}$ ). Six days post-transfection conditioned medium containing recombinant protein was harvested by low-speed centrifugation (10 minutes, $1000 \mathrm{~g}$ ) followed by high-speed centrifugation (10 minutes, 4000g) and immobilized metal affinity chromatography (IMAC) purification. The VHH was further purified by gel filtration using a Superdex75 26/600 column. The resulting VHHcontaining fractions were sterilized by filtration over a $0.22 \mu \mathrm{m}$ syringe filter and stored at $4{ }^{\circ} \mathrm{C}$.

\section{Assay performance studies Specificity}

The assay was performed as described under "Active VWF immunosorbent assay" in the Methods section. Normal pooled plasma (NPP, static or vortexed at 2,500 rpm for 10 minutes), HVWF, R1306W VWF or R1306Q VWF (all $1.7 \mathrm{ug} / \mathrm{mL}$ ), were serially diluted (except for vortexed NPP, only measured at 1:10 dilution) with dilution buffer (PBS/1\% BSA) and incubated in the plate coated with VHH at RT for 2 hours, followed by detection steps as described.

\section{Precision}

Pools of citrated plasma samples were prepared based on previously measured active VWF levels: the "low" (L) pool contained samples with active VWF levels between 91 and 110\% (mean 102\%), the "medium" (M) pool contained samples with active VWF levels between 150 and 200\% (mean 182\%) and the "high" pool samples with active VWF levels $>220 \%$ (mean 235\%). These "cut-offs" were arbitrarily chosen, to cover the range of relevant values, and they do not have clinical meaning. Intra-assay (within run) precision was assessed by repeated $(n=20)$ measurements of these plasma pools active VWF in one plate on the same day. Inter-assay (between run) precision was determined by duplicate measurements of the same plasma pools on 20 different days, within 2 months. Acceptance criteria were CV $\leq 10 \%$ for intra-assay precision and CV $\leq 15 \%$ for inter-assay precision.

\section{Accuracy}

Accuracy was assessed by adding known concentrations of recombinant R1306W VWF (stock $17 \mu \mathrm{g} / \mathrm{mL}$, concentrations 1.0, 0.75, 0.5, 0.35 or $0.25 \mu \mathrm{g} / \mathrm{mL}$ R1306W VWF) or 
dilution buffer (control) to NPP. Recovery (Rec\%) was calculated based on the observed $\mathrm{OD}\left(\mathrm{O}_{\mathrm{OD}}\right)$ and expected OD $\left(\mathrm{E}_{\mathrm{OD}}\right)$ as follows: Rec\% $=\left(\mathrm{O}_{\mathrm{OD}} / \mathrm{E}_{\mathrm{OD}}\right) * 100 \%$ and was considered acceptable between $90 \%$ and $110 \%$.

\section{Linearity}

Linearity was determined by two serial recovery studies. First, for spiking with recombinant R1306W VWF, a pool of plasma with low active VWF was prepared. Half of this low active VWF pool was spiked with recombinant R1306W VWF (maximal 10 V/V\%, not higher to minimize matrix dilution effects) to obtain a high active VWF pool. The high active VWF sample pool was then diluted with the remaining low active VWF sample pool to create a 12 steps 1.2-fold dilution series. Secondly, to determine the linearity when diluting native plasma samples, a high pool (active VWF $>200 \%$ of NPP, as determined in previous experiments) was diluted with a low pool (active VWF $<120 \%$, as determined in previous experiments) in 5 steps: $100 \%$ of each and 3 mixes of $75 \% / 25 \%, 50 \% / 50 \%$ and $25 \% / 75 \%$. For both dilution experiments three replicates per dilution step were measured in 1 run.

\section{Limits}

The limit of quantitation (LoQ) was determined using 11 citrated plasma pools over a range of concentrations (114 - 248\%), each measured in duplicate over 10 days. The LoQ was extrapolated from the binomial curve through the points in the plot of mean active VWF (X-axis) versus inter-assay CV\% (y-axis) as the mean active VWF level at which the inter-assay CV was 20\%. The limit of blank (LoB) was determined by measuring a blank sample (dilution buffer) 60 -fold and calculated as LoB=mean blank $+1.645 * \mathrm{SD}_{\text {blank }}$. The limit of detection (LOD) was determined based on the LoB and 60 test replicates of a sample known to contain a low concentration of analyte (also used for intra-assay precision as " low/L" pool). LoD was calculated as LoD=LoB+1.645(SD low sample $_{\text {). }}$.

\section{Stability}

We performed a stability study limited to the conditions used for (the validation of) this assay, i.e. with the aims to assess the (1) effect of repeated freeze/thawing and (2) stability during storage at $-80^{\circ} \mathrm{C}$. To determine freeze/thaw stability, a plasma pool with medium active VWF level (152.9\%) was stored frozen at $-80{ }^{\circ} \mathrm{C}$ and was measured in triplicate

with the immunosorbent assay after being subjected to four freeze-thaw cycles during one day. Furthermore, at day 1, day 2, day 7,1 month and 2 months aliquots of this pool were thawed and active VWF was determined in triplicate. Mean recovery of active VWF expressed in absolute values (i.e., absolute recovery, expressed as active VWF in \% of NPP) and in percent of the baseline value (i.e., relative recovery) was calculated. The acceptance criterion was $\pm 10 \%$ difference from the original concentration. 


\section{Flow cytometric analysis of VWF:platelet binding}

Test strips for three test conditions (no agonist control, $1.2 \mathrm{mg} / \mathrm{mL}$ ristocetin and positive beads control $[\mathrm{PC}]$ ) were prepared in advance and stored at $-20^{\circ} \mathrm{C}$. The reaction mixtures with a total volume of $20 \mu \mathrm{l}$ consisted of $2 \mu \mathrm{l}$ FITC-conjugated anti-VWF antibodies (CL7616F, Cedarlane, Burlington, USA) and $0.5 \mu \mathrm{l}$ APC-conjugated anti-CD41a antibodies (559777, BD Biosciences, San Jose, USA) with or without agonist in HEPESbuffered saline (HBS, $10 \mathrm{mmol} / \mathrm{L}$ HEPES, $150 \mathrm{mmol} / \mathrm{L} \mathrm{NaCl}, 1 \mathrm{mmol} / \mathrm{L} \mathrm{MgSO}{ }_{4}, 5 \mathrm{mmol} / \mathrm{L}$ $\mathrm{KCL}, \mathrm{pH}$ 7.4). The PC tube only contained $2 \mu \mathrm{F}$ FITC-conjugated anti-VWF antibodies in HBS.

After venipuncture, whole blood was kept at RT for at least 30 minutes and subsequently incubated at $37^{\circ} \mathrm{C}$ for 10 minutes. All tests were performed at $37^{\circ} \mathrm{C}$. Blood was diluted $1: 4$ in HBS. From the diluted blood, $5 \mu \mathrm{l}$ was added to the tubes with reaction mixture $(20 \mu \mathrm{l}$, final dilution 1:20). In addition, $5 \mu$ l of anti-mouse Ig k particles was added to the PC tube. Tests were incubated for exactly 20 minutes at $37^{\circ} \mathrm{C}$. Reactions were stopped by adding $250 \mu \mathrm{l}$ fixation solution $\left(137 \mathrm{mmol} / \mathrm{L} \mathrm{NaCl}, 2.7 \mathrm{mmol} / \mathrm{L} \mathrm{KCl}, 1.12 \mathrm{mmol} / \mathrm{L} \mathrm{NaH}_{2} \mathrm{PO}_{4^{\prime}} 1.15\right.$ $\mathrm{mmol} / \mathrm{L} \mathrm{KH}_{2} \mathrm{PO}_{4^{\prime}} 10.2 \mathrm{mmol} / \mathrm{L} \mathrm{Na}_{2} \mathrm{HPO}_{4} 4 \mathrm{mmol} / \mathrm{L}$ EDTA, 0.5\% formaldehyde). Samples were analysed 1 day after the experiment. Flow cytometry was used to discriminate platelets from other cells, using the forward and side scatter pattern and by gating on the CD41a positive cells. Median fluorescent intensity in the FITC gate was selected to determine VWF-platelet binding. Results are corrected for the MFI in the unstimulated test condition and expressed as a \% of the MFI for the PC beads.

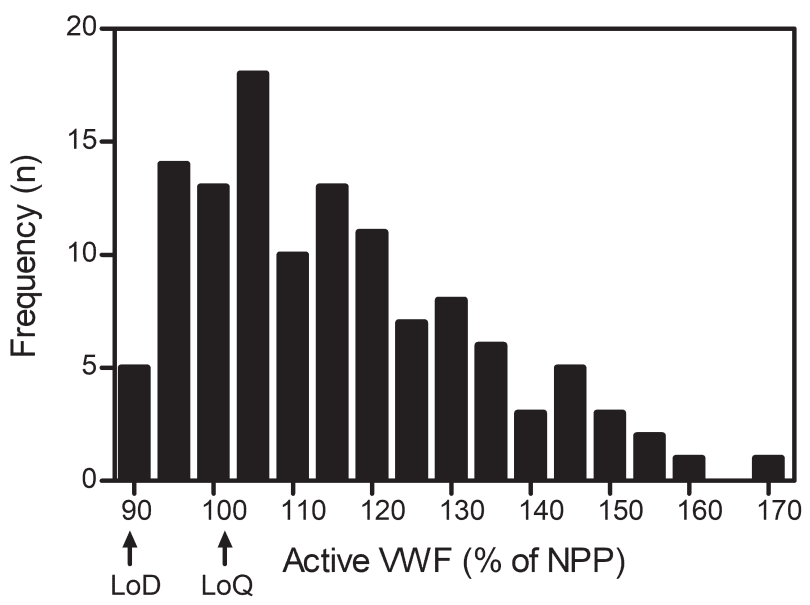

S1 Fig. Distribution of active VWF in a healthy population. Citrated plasma samples obtained from 120 healthy subjects were measured with the active VWF assay. Limit of detection (LoD) and Limit of Quantitation (LoQ) are indicated. The frequency distribution shows skewness to the right. 


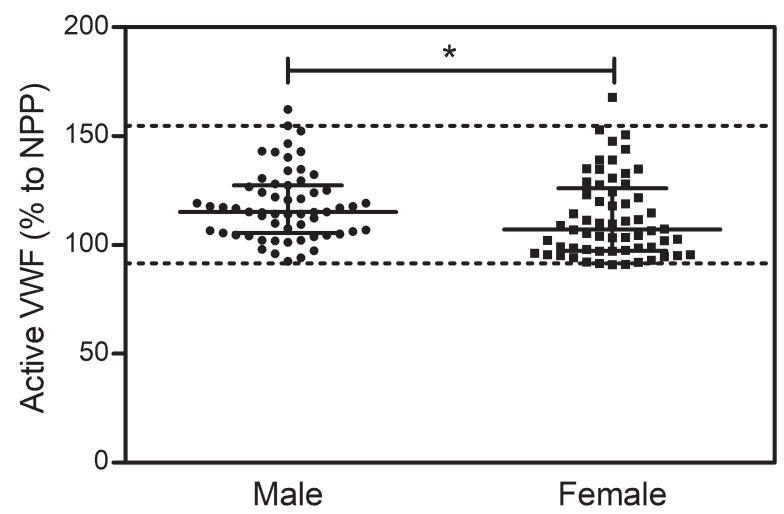

S2 Fig. Effect of gender on active VWF levels. Active VWF levels (normalized to levels in NPP) were determined in plasma of 120 healthy volunteers, and are shown here for males $(n=60)$ and females $(n=60)$. Median and IQR are indicated. The area delineated by the dot- ted lines represents the reference interval of the total population (2.5 percentile- 97.5 percentile). Active VWF levels were significantly ( $p=0.033$ ) higher in males compared to females, but adjustment for VWF:Ag levels abolished the association between active VWF and gender.
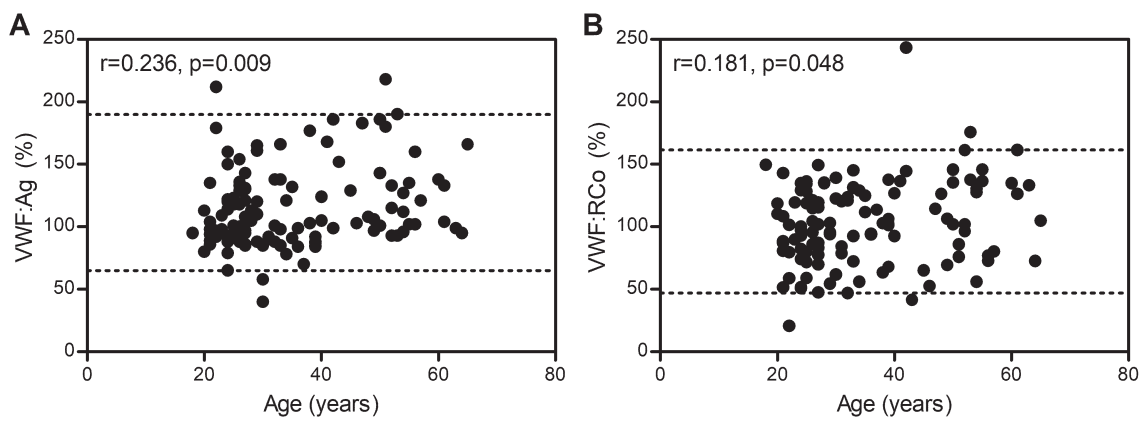

S3 Fig. VWF:Ag and VWF:RCo correlate significantly with age. Scatterplot and correspond- ing Spearman rank correlation ( $r$ ) between VWF:Ag (a) and VWF:RCo (b) with age. Dotted lines delineate reference intervals for VWF:Ag and VWF:RCo determined in this study. 

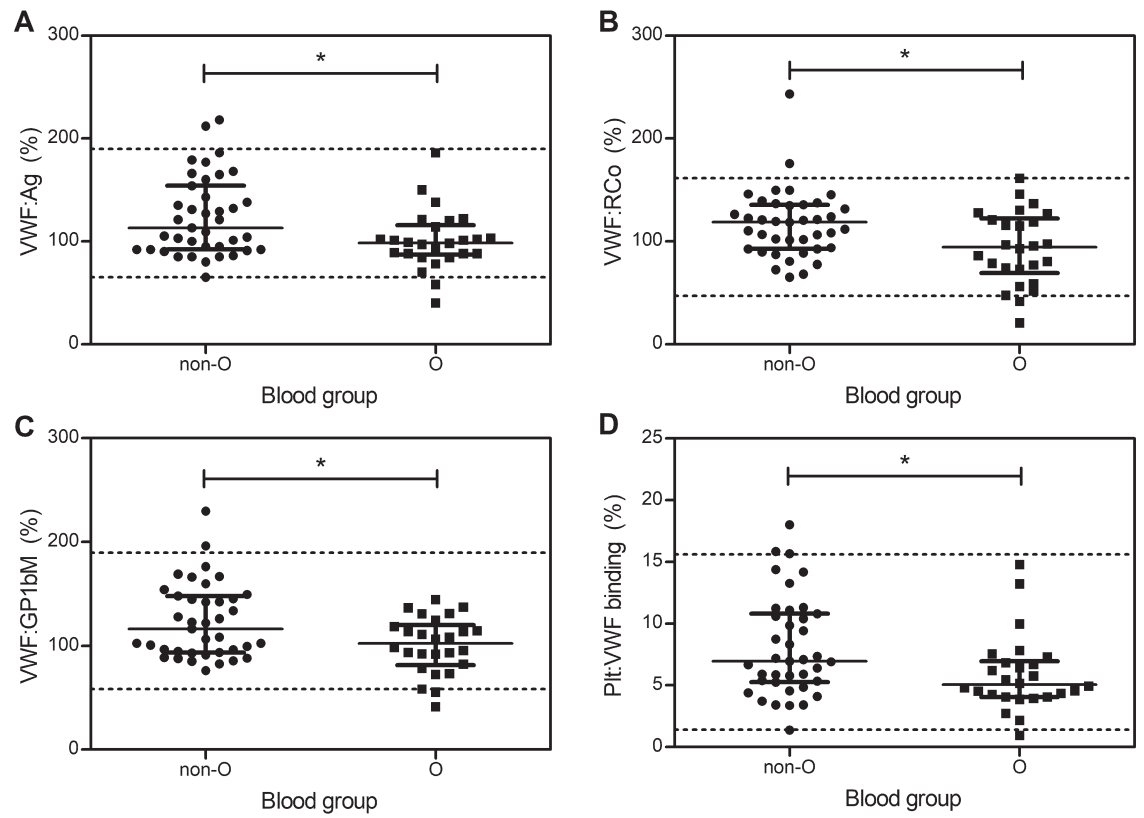

S4 Fig. Effect of blood group $O$ and non-O on VWF parameters. VWF:Ag (A), VWF:RCo (B), VWF:GP1bM (C) and Plt:VWF binding (D) were determined in plasma of 120 healthy volunteers, and are shown here for individuals with non-O and O blood group. Median and IQR are indicated. The areas delineated by the dotted lines represent the reference intervals (2.5 percentile- 97.5 percentile). Statistical significance of differences in VWF parameters between $\mathrm{O}$ and non-O subjects were tested by Mann-Whitney $U$ test, $p<0.05$.

S1 Table. Spearman rank correlations between VWF assays

\begin{tabular}{|c|c|c|c|c|c|c|}
\hline Assay & VWF:Act & VWF:Ag & VWF:RCo & VWF:GP1bM & VWFpp & Plt:VWF \\
\hline \multicolumn{7}{|l|}{ VWF:Act } \\
\hline VWF:Ag & $0.390 * \star$ & & & & & \\
\hline VWF:RCo & $0.401^{\text {** }}$ & $0.617^{* \star}$ & & & & \\
\hline VWF:GP1bM & $0.464^{* *}$ & $0.669 * *$ & $0.564 * *$ & & & \\
\hline VWFpp & $0.281^{\text {** }}$ & 0.457 ** & 0.442 ** & $0.404 * *$ & & \\
\hline Plt:VWF & 0.273 ** & 0.636 ** & 0.619 ** & 0.679 ** & $0.396 * *$ & \\
\hline
\end{tabular}

Values represent Spearman rank correlation coefficients $(r)$ with corresponding significance: $* \star, p$ value <0.01. VWF:Act, active VWF; VWF:Ag, VWF antigen; VWF:RCo, VWF ristocetin cofactor activity; VWF:GP1bM, VWF binding to gain-of-function GP1b fragments; VWFpp, VWF propeptide; Plt:VWF, platelet VWF binding. 


\section{REFERENCES}

1. Hulstein JJ, de Groot PG, Silence K, Veyradier A, Fijnheer R, Lenting PJ: A novel nanobody that detects the gain-of-function phenotype of von Willebrand factor in ADAMTS13 deficiency and von Willebrand disease type 2B. Blood 2005, 106(9):3035-3042. 



\section{0}

Summary, discussion and prospects 


\section{GENERAL DISCUSSION}

This thesis focuses on the interplay between platelets and coagulation and the application of flow cytometric platelet function tests in clinical settings. Chapter 2 describes a new mechanism to initiate thrombin generation via GPVI activated platelets. In chapter 3 the interplay between platelets and coagulation is investigated in multiple myeloma patients (MM) and healthy controls. In chapter 4, 5 and 6 clinical studies are presented investigating platelet reactivity/activation in patients with chronic obstructive pulmonary disease (COPD), patients on dual anti-platelet therapy (DAPT) and in patients with suspected thrombocytopathy. Chapter 7, the flow cytometric platelet function test is used to study platelet-VWF binding and is compared to an in-house ELISA assay for measuring circulating active VWF.

\section{Coagulation initiation by GPVI activated platelets}

Established platelet derived triggers of coagulation are secreted polyphosphates (PolyP) to activate FXII and the expression of functional tissue factor (TF), which remains controversial $[1,2]$. Collagen can also trigger coagulation via FXII activation, however, we showed in the current thesis, that, in the presence of platelets (in platelet-rich plasma), coagulation initiated by collagen can only partly be antagonized by a FXIla inhibitor. We hypothesized that activated platelets play a role in the initiation of coagulation by collagen and tried to investigate the underlying mechanisms.

We found that, not only collagen, but also other GPVI agonists (CRP-XL and convulxin) can induce platelet activated thrombin generation (TG), which is mainly displayed as a moderate onset of TG with a high peak. Experiments with inhibitors and factor deficient plasmas suggested that initiation of TG via GPVI activated platelets is not dependent on plasma FXII, FXI or TF/FVIIa. In a purified system with washed platelets, FX and FVIII, results suggested that activated platelets deliver FIXa, which was later confirmed by the inhibitory effect of an anti-FIX Ab and the recovery of TG in FIX deficient plasma supplemented with FVIII.

It was shown with transmission electron microscopy study that FIX is found in the alpha granules of platelets. The origin of the FIXa activity from activated platelets is not known. It is possible that FIX is produced in Megakaryocytes, but it is also possible FIX is taken from the plasma and transported to the alpha granules [3]. In this thesis, we could not confirm the presence of FIX with western blot or with protein fingerprinting, indicating that the concentrations are extremely low.

Other possibilities for initiation of coagulation by activated platelets were excluded. For example, polyP, an established trigger of FXII activation which is stored in dense 
granules, was not the trigger of coagulation in our experiments as TG in CTI-inhibited and FXII-deficient plasma was not affected. In addition, we excluded that TG was induced by platelet derived FVII(a) or FXIa as FVIIai nor FVIla or FXI inhibiting antibodies had a significant effect.

\section{Platelets and coagulation in treated multiple myeloma patients}

Multiple myeloma (MM) is a disease characterized by malignant proliferation of plasma cells that produce a monoclonal immunoglobulin, called M protein or paraprotein. In recent years, with increasing clinical evidence and the development of treatment, venous thromboembolism (VTE) and its prevention seem to gain more attention in MM patients as both bleeding and VTE are recognised complications of MM [4]. There are multiple factors that can contribute to a high thrombotic risk in MM patients. First of all, cancer and cancer treatments in general are identified as risk factors for VTE [5]. In patients with MM, the treatment with immunomodulatory drugs like thalidomide or lenalidomide in combination with high dosage of dexamethasone, doxorubicin, or combination chemotherapy are shown to be associated with high VTE rates [6]. Secondly, the advanced age of most MM patients that is associated with immobility and/ or hyperviscosity results in increased VTE risk $[7,8]$. Thirdly, activation of coagulation, reduced natural anticoagulation mechanisms and an inflammatory environment in MM can attribute to a hypercoagulable state [9-11]. On the other hand, MM itself belongs to a broader disease category called monoclonal gammopathies, which often predispose patients to haemorrhage, especially following surgical procedures. Possible underlying mechanisms like thrombocytopenia, paraprotein-induced platelet function defects, acquired von Willebrand syndrome, acquired factor $X$ deficiency, abnormalities of the function of fibrin can be the cause $[12,13]$.

We investigated the complex situation of higher incidence of haemostatic abnormalities in on-treatment MM patients by measuring their platelet reactivity and activity and coagulation profiles. The major strength of our study is measuring TG in the presence of blood cells. A delayed initiation of TG (lagtime and time-to peak were prolonged) and increased thrombin potential (increased ETPp) was measured in whole blood (WB) of MM patients, which was associated with properties of RBCs and platelets (negative correlation between RBC count and platelet reactivity, and the initiation time of WB-TG). This imbalanced haemostasis may explain that MM patients have a high incidence of VTE and a high bleeding tendency. Interestingly, in contrast to WB-TG, in the absence of cells (plasma TG), no difference in TG was measured between MM patients and controls. MM patients had impaired allbß3 activation in response to agonists, while baseline platelet activation and platelet-monocyte complexes were comparable with the control group. Our platelet reactivity results point in the same direction as previous research that described platelet hypo-reactivity and reduced aggregation in MM patients [14, 
15]. There are several explanations for platelet hypo-reactivity in MM. First of all, the nonspecific coating by paraproteins is supposed to inhibit platelet function [16], and the association between impaired platelet function (based on prolonged bleeding time and abnormal platelet aggregation) and elevated serum paraproteins was reported [17, 18]. Secondly, high activation of platelets may result in "platelet exhaustion", which is then displayed as low responsiveness [19]. Furthermore, besides the cause from the disease itself, anti-myeloma therapy may also influence platelet vitality, as one study reported platelet dysfunction in thrombocytopenic cancer patients undergoing chemotherapy [20].

A strong point of the study is the combined invitation of patients and their controls. This guarantees the identical preconditioning of the blood samples. Furthermore, the controls were of the same age and the same social economic state, which make the groups comparable.

The interesting observations on the differences between plasma TG and whole blood TG, and the correlations between blood cell properties and whole blood TG parameters give insights in the involvement of blood cells in thrombin generation under pathological conditions. Also this study may trigger future prospective studies on MM related hemostasis disorders to further study the impact of whole blood thrombin generation on the incidence of thrombosis and bleeding in MM patients.

\section{Acute exacerbations of COPD induce a prothrombotic state}

Chronic obstructive pulmonary disease (COPD) is characterized by airway inflammation, excessive mucus production, progressive destruction of the lung parenchyma with loss of elasticity and abnormal remodelling of the pulmonary vasculature [22]. Patients with COPD are reported to have increased risk for cardiovascular events, like myocardial infarction, stroke and pulmonary embolism, particularly following an acute exacerbation [23-25]. Previous studies have observed that systemic inflammation may contribute to platelet activation. With the extensive crosstalk between inflammation and coagulation, we hypothesized that the elevated inflammatory state during an AE-COPD may contribute to a prothrombotic phenotype $[26,27]$.

In chapter 4, in a cohort of 32 COPD patients, we showed that platelet activation measured as the levels of platelet-monocyte complexes (PMCS), was significantly increased during AE-COPD compared to day 3 of treatment and convalescence. The main interactions between platelets and monocytes to form PMCs are via binding of platelet P-selectin to PSGL-1 on monocytes or by binding of monocyte Mac-1 to platelet allbß3 or GPIba. Interestingly, P-selectin expression and allbß3 activation did not change and MAC-1 expression increased during an AE-COPD suggesting that PMC formation is driven by 
monocyte activation (binding of MAC-1 to platelet GP1ba). MAC-1 expression can be driven by inflammatory mediators such as IL-6 and CRP, and we found associations between CRP level (marker for systemic inflammation) with both platelet activation level and platelet reactivity level. Concomitant with elevated platelet activation, we saw significantly enhanced plasmatic coagulation (the TG peak, ETP and velocity were significantly increased) and higher level of VWF (both antigen and the active form) during AE-COPD, all together suggesting a pro-thrombotic state. D-dimer levels and the time until clot formation did not change during AE-COPD.

Our results on platelet activation are in line with previous published data reporting higher circulating platelet-monocyte aggregates in patients with stable COPD compared to well-matched controls, and also further rise of these complexes during an acute exacerbation [28]. In contrast to Munoz-Esquerre et al. who reported an increased platelet reactivity during AECOPD, we didn't observe a significant difference in platelet reactivity in our cohort. Possible explanations are the differences of sample size, patient inclusion criteria and measurement methods between studies [29]. Due to the small sample size of our study with some loss to follow-up and with only 3 patients suffering from a cardiovascular event during investigation, we were not able to find direct links between clinical outcomes and markers of haemostasis.

Apart from this, our results seem to give more evidence on the interwoven connection between inflammation and platelets in COPD patients. Increased systemic inflammation, hypoxemia and oxidative stress are reported to induce platelet activation in these patients $[30,31]$. In the meantime, increasing evidence suggests that platelets exert significant modulatory effects on inflammatory- and immune-mediated pathways, for instance, through an increase in leukocyte elastase activity and the dysregulation of specific hypoxia-related signalling pathways $[29,32]$. Though not in the context of treating COPD, the use of $\mathrm{P}_{2} \mathrm{Y}_{12}$ inhibitors was found to decrease platelet-monocyte interaction as well as systemic inflammation [33, 34]. Furthermore, the use of antiplatelet agents was associated with a significant reduction in all-cause mortality in COPD patients [35] and anticoagulant low-molecular-weight heparin (LMWH), recommended by current guidelines for VTE prevention in AE-COPD patients with reduced mobility, can have antiinflammatory properties [36-38].

Taken together, our study demonstrates that AE-COPD patients with systemic inflammation have an increased prothrombotic tendency and that they may benefit from anti-thrombotic treatment. 


\section{WB-PACT assay for recognizing platelet function defects and assessing anti- platelet therapy efficacy}

Previously our lab optimized and standardized a fast, user-friendly WB-PACT assay, together with the development of platelet-VWF binding measurement in the same setting [39]. In chapter 5 and 6, the WB-PACT test was compared with light transmission aggregometry (LTA) to study a group of patients on dual antiplatelet therapy and patients suspected of bleeding diathesis, respectively.

Platelet function defects (PFDs) may occur because of inherited or acquired conditions and sometimes it coexist with thrombocytopenia [40]. Inherited platelet function disorders (IPFDs) are a group of bleeding disorders, characterized by a wide phenotypic and genotypic heterogeneity. Severe IPFD, like Glanzmann Thrombasthenia (GT) or Bernard-Soulier Syndrome (BSS), are straightforward to identify, while the diagnosis of most other mild forms is cumbersome and requires complex assays, which makes PFDs underdiagnosed, and its prevalence underestimated in some sense [41-45]. More common than IPFDs in clinical practice, acquired platelet function disorders can be due to some systemic/hematologic disorders or adverse reactions after procedures, or more often it can be the anticipated effects of antiplatelet drugs aiming for thrombosis prevention [46].

For diagnosing PFDs, the Scientific and Standardization Committee (SSC) guidelines of the International Society on Thrombosis and Haemostasis (ISTH) recommend an algorithm flow chart. The first step in this chart is the evaluation of personal, familial bleeding history and bleeding score, followed by a step-wise laboratory investigation, which contains preliminary laboratory investigations (full blood count, prothrombin time, activated partial thromboplastin time and VWF screening tests) and later a diagnostic work-up that includes a blood smear, LTA and flow cytometric measurements [47].

In chapter 6 we introduced a scoring system for the WB-PACT and in a population of 161 patients suspected of bleeding diathesis, a moderate agreement was found between the WB-PACT and LTA. Moreover, in patients with an elevated ISTH bleeding score, WB-PACT detected more abnormal responses than LTA, and the flow cytometric test to detect VWF-platelet binding detected some patients with deviations in VWF concentration or function.

The partial agreement between LTA and WB-PACT in this study can be due to the different methodology (LTA is semi-quantitative while WB-PACT gives quantitative results), the scoring system (the cut off values can largely affect the analysis) and differences in agonists used (LTA was not performed with PAR-1 agonist, while collagen, epinephrine and arachidonic acid were not included in WB-PACT). Previously by only comparing the 
responses after ADP and CRP-XL stimulations, Navred et al. also reported a moderate agreement between their modified flow cytometric platelet activation and LTA in detection of PFDs [48]. Although the partial agreement is to some extent expected, our results still offer some information on cross-validation between the two tests. By analysing the associations of the tests with the ISTH bleeding score, we suggested that WB-PACT may have added value for the routine diagnostic work-up in patients who need to have platelet function tested while others suggest that flow cytometric analysis of platelet function have potential to become an alternative for LTA [49].

In chapter 5, we assessed platelet function, or to be more precise, in vitro residual platelet reactivity by LTA and WB-PACT in 42 patients receiving aspirin and clopidogrel. Aspirin and clopidogrel are the two most widely used antiplatelet drugs for secondary prevention of cardiovascular diseases in the world. However, some patients do not respond adequately to aspirin or to clopidogrel [50-54]. High on-treatment residual platelet reactivity (HRPR) was reported to be associated with high risk of thrombotic events [55]. This argues for, in vitro, platelet function monitoring of the effects of antiplatelet drugs. In this small population, a moderate agreement was found between MesADP-triggered WB-PACT and ADP-triggered LTA on recognizing HRPR. As arachidonic acid was not included in the WB-PACT agonist panel, we were unable to compare the detection of aspirin resistance. Nonetheless, this study may offer some information for future investigations on the application of WB-PACT in anti-platelet therapy monitoring.

\section{Active VWF detection by ELISA}

In chapter 7, we describe the technical validation of an in-house developed immunosorbent assay with a VHH directed against a cryptic epitope of the VWF A1 domain. If VWF undergoes a conformational change due to activation, then the cryptic domain will become available for the $\mathrm{VHH}$ and thus measurable by the ELISA. A similar ELISA for detecting active VWF already existed for more than 10 years, however technical validation for broader applications was lacking [56]. The specificity, the intra- and inter assay variability and the linearity of this assay was demonstrated and a reference interval for active VWF in 120 healthy adults was established. Besides, positive correlations between active VWF and other VWF parameters (quantifications for different forms of VWF and qualitative evaluation of its binding capacity) were found in this healthy population.

Previously, it has been shown that active VWF levels are associated with VWD type 2B, HELLP, malaria and diabetes [56-59]. Furthermore, active VWF was recommended for stratifying SIRS patients at high mortality risk with the APACHE-IV score and as an independent risk factor for first STEMI in patients [60, 61]. After the validation study, this assay was applied in several studies, in which increased circulating active VWF levels are 
found in healthy individuals following strenuous exercise, patients with chronic kidney disease and AE-COPD patients [62-64]. With a robust analytical performance and a high specificity for the active form of VWF, the assay can be used to measure active VWF as a biomarker in many pathologies.

\section{CONCLUSION}

In conclusion, the current thesis describes novel insights in platelet activation mediated initiation of coagulation. The studies in this thesis confirm that blood cells are crucial players in the regulation of coagulation, especially in pathological disorders. Blood cell dependent thrombin generation is affected in $\mathrm{MM}$, while plasma thrombin generation seems not associated with MM prevalence. Whether WB-TG can predict the increased bleeding and thrombosis risk in MM-patients needs to be studied in a cohort study on MM patients and thrombosis incidence during follow-up. This thesis shows that platelet activation and platelet monocyte complex formation are affected during the acute exacerbation of COPD. Furthermore, WB-PACT is showed to have value for monitoring patients on DAPT and recognizing patients with bleeding diathesis. 


\section{REFERENCES}

1. Panes O, Matus V, Saez CG, Quiroga T, Pereira J, Mezzano D: Human platelets synthesize and express functional tissue factor. Blood 2007, 109(12):5242-5250.

2. Smith SA, Mutch NJ, Baskar D, Rohloff P, Docampo R, Morrissey JH: Polyphosphate modulates blood coagulation and fibrinolysis. Proceedings of the National Academy of Sciences of the United States of America 2006, 103(4):903-908.

3. Berger G, Massé JM, Cramer EM: Alpha-granule membrane mirrors the platelet plasma membrane and contains the glycoproteins Ib, IX, and V. Blood 1996, 87(4):1385-1395.

4. Eby C: Pathogenesis and management of bleeding and thrombosis in plasma cell dyscrasias. British journal of haematology 2009, 145(2):151-163.

5. Geerts WH, Bergqvist D, Pineo GF, Heit JA, Samama CM, Lassen MR, Colwell CW: Prevention of venous thromboembolism: American College of Chest Physicians Evidence-Based Clinical Practice Guidelines (8th Edition). Chest 2008, 133(6 Suppl):381s-453s.

6. Palumbo A, Rajkumar SV, Dimopoulos MA, Richardson PG, San Miguel J, Barlogie B, Harousseau J, Zonder JA, Cavo M, Zangari M et al: Prevention of thalidomide- and lenalidomide-associated thrombosis in myeloma. Leukemia 2008, 22(2):414-423.

7. Anderson KC, Alsina M, Bensinger W, Biermann JS, Chanan-Khan A, Cohen AD, Devine S, Djulbegovic B, Gasparetto C, Huff CA et al: NCCN clinical practice guidelines in oncology: multiple myeloma. Journal of the National Comprehensive Cancer Network : JNCCN 2009, 7(9):908-942.

8. Scates SM: Diagnosis and treatment of cancer-related thrombosis. Hematology/oncology clinics of North America 1992, 6(6):1329-1339.

9. Zangari M, Elice F, Fink L, Tricot G: Thrombosis in multiple myeloma. Expert review of anticancer therapy 2007, 7(3):307-315.

10. Rickles FR, Patierno S, Fernandez PM: Tissue factor, thrombin, and cancer. Chest 2003, 124(3 Suppl):58s-68s.

11. Talamo GP, Ibrahim S, Claxton D, Tricot GJ, Fink LM, Zangari M: Hypercoagulable states in patients with multiple myeloma can affect the thalidomide-associated venous thromboembolism. Blood coagulation \& fibrinolysis : an international journal in haemostasis and thrombosis 2009, 20(5):337-339.

12. Glaspy JA: Hemostatic abnormalities in multiple myeloma and related disorders. Hematology/oncology clinics of North America 1992, 6(6):1301-1314.

13. Carr ME, Jr., Zekert SL: Abnormal clot retraction, altered fibrin structure, and normal platelet function in multiple myeloma. The American journal of physiology 1994, 266(3 Pt 2):H1195-1201.

14. Cieslar P, Masova L, Scheiner T, Rysava J, Krizova P, Danzigova Z, Spicka I, Tesar V: Oxidative stress and platelet function in multiple myeloma and renal insufficiency: clinical relations of different tests. Thrombosis research 2002, 105(4):277-283.

15. Egan K, Cooke N, Dunne E, Murphy P, Quinn J, Kenny D: Platelet hyporeactivity in active myeloma. Thrombosis research 2014, 134(3):747-749.

16. Djunic I, Elezovic I, Vucic M, Srdic-Rajic T, Konic-Ristic A, llic V, Milic N, Bila J, Suvajdzic-Vukovic N, Virijevic M et al: Specific binding of paraprotein to platelet receptors as a cause of platelet dysfunction 
in monoclonal gammopathies. Acta haematologica 2013, 130(2):101-107.

17. Perkins HA, Mackenzie MR, Fudenberg HH: Hemostatic defects in dysproteinemias. Blood 1970, 35(5):695-707.

18. Penny R, Castaldi PA, Whitsed HM: Inflammation and haemostasis in paraproteinaemias. British journal of haematology 1971, 20(1):35-44.

19. O'Sullivan LR, Meade-Murphy G, Gilligan OM, Mykytiv V, Cahill MR, Young PW: Platelet hyperactivation and hyporesponsiveness at diagnosis in multiple myeloma persists during treatment initiation. Thromb Res 2021, 203:186-189.

20. Baaten C, Moenen F, Henskens YMC, Swieringa F, Wetzels RJH, van Oerle R, Heijnen HFG, Ten Cate H, Holloway GP, Beckers EAM et al: Impaired mitochondrial activity explains platelet dysfunction in thrombocytopenic cancer patients undergoing chemotherapy. Haematologica 2018, 103(9):15571567.

21. Rabe KF, Watz H: Chronic obstructive pulmonary disease. Lancet (London, England) 2017, 389(10082):1931-1940.

22. Donaldson GC, Hurst JR, Smith CJ, Hubbard RB, Wedzicha JA: Increased risk of myocardial infarction and stroke following exacerbation of COPD. Chest 2010, 137(5):1091-1097.

23. Reilev M, Pottegard A, Lykkegaard J, Sondergaard J, Ingebrigtsen TS, Hallas J: Increased risk of major adverse cardiac events following the onset of acute exacerbations of COPD. Respirology 2019, 24(12):1183-1190.

24. Rothnie KJ, Yan R, Smeeth L, Quint JK: Risk of myocardial infarction (MI) and death following MI in people with chronic obstructive pulmonary disease (COPD): a systematic review and meta-analysis. BMJ open 2015, 5(9):e007824.

25. Hansson GK: Inflammation, atherosclerosis, and coronary artery disease. The New England journal of medicine 2005, 352(16):1685-1695.

26. Libby P: Inflammation in atherosclerosis. Nature 2002, 420(6917):868-874.

27. Maclay JD, McAllister DA, Johnston S, Raftis J, McGuinnes C, Deans A, Newby DE, Mills NL, MacNee W: Increased platelet activation in patients with stable and acute exacerbation of COPD. Thorax 2011, 66(9):769-774.

28. Munoz-Esquerre M, Ferreiro JL, Huertas D, Marcano AL, Lopez-Sanchez M, Roura G, Gomez-Hospital JA, Dorca J, Cequier A, Santos S: Impact of acute exacerbations on platelet reactivity in chronic obstructive pulmonary disease patients. Int J Chron Obstruct Pulmon Dis 2018, 13:141-148.

29. Vaidyula VR, Boden G, Rao AK: Platelet and monocyte activation by hyperglycemia and hyperinsulinemia in healthy subjects. Platelets 2006, 17(8):577-585.

30. Diodati JG, Cannon RO, 3rd, Epstein SE, Quyyumi AA: Platelet hyperaggregability across the coronary bed in response to rapid atrial pacing in patients with stable coronary artery disease. Circulation 1992, 86(4):1186-1193.

31. Robert L, Labat-Robert J: Platelets, Micro-Particles and Elastase. A Review with Extrapolation to the Mechanism of Generation and Bio-Pathology of Platelet Fragments. Pathology oncology research : POR 2017, 23(2):455-458.

32. Frelinger AL, 3rd, Jakubowski JA, Li Y, Barnard MR, Linden MD, Tarnow I, Fox ML, Sugidachi A, Winters 
$\mathrm{KJ}$, Furman Ml et al: The active metabolite of prasugrel inhibits adenosine diphosphate- and collagenstimulated platelet procoagulant activities. Journal of thrombosis and haemostasis : JTH 2008, 6(2):359365.

33. Braun OO, Johnell M, Varenhorst $\mathrm{C}$, James $\mathrm{S}$, Brandt JT, Jakubowski JA, Winters KJ, Wallentin L, Erlinge D, Siegbahn A: Greater reduction of platelet activation markers and platelet-monocyte aggregates by prasugrel compared to clopidogrel in stable coronary artery disease. Thrombosis and haemostasis 2008, 100(4):626-633.

34. Pavasini R, Biscaglia S, d'Ascenzo F, Del Franco A, Contoli M, Zaraket F, Guerra F, Ferrari R, Campo G: Antiplatelet Treatment Reduces All-Cause Mortality in COPD Patients: A Systematic Review and Meta-Analysis. Copd 2016, 13(4):509-514.

35. Qian Y, Xie H, Tian R, Yu K, Wang R: Efficacy of low molecular weight heparin in patients with acute exacerbation of chronic obstructive pulmonary disease receiving ventilatory support. Copd 2014, 11(2):171-176.

36. Ludwig RJ: Therapeutic use of heparin beyond anticoagulation. Current drug discovery technologies 2009, 6(4):281-289.

37. Schünemann HJ, Cushman M, Burnett AE, Kahn SR, Beyer-Westendorf J, Spencer FA, Rezende SM, Zakai NA, Bauer KA, Dentali F et al: American Society of Hematology 2018 guidelines for management of venous thromboembolism: prophylaxis for hospitalized and nonhospitalized medical patients. Blood Adv 2018, 2(22):3198-3225.

38. Huskens D, Sang Y, Konings J, van der Vorm L, de Laat B, Kelchtermans H, Roest M: Standardization and reference ranges for whole blood platelet function measurements using a flow cytometric platelet activation test. PloS one 2018, 13(2):e0192079.

39. Cattaneo M: Inherited platelet-based bleeding disorders. Journal of thrombosis and haemostasis: JTH 2003, 1(7):1628-1636.

40. Gresele P, Bury L, Falcinelli E: Inherited Platelet Function Disorders: Algorithms for Phenotypic and Genetic Investigation. Seminars in thrombosis and hemostasis 2016, 42(3):292-305.

41. Hayward CP, Rao AK, Cattaneo M: Congenital platelet disorders: overview of their mechanisms, diagnostic evaluation and treatment. Haemophilia: the official journal of the World Federation of Hemophilia 2006, 12 Suppl 3:128-136.

42. Quiroga T, Goycoolea M, Panes O, Aranda E, Martínez C, Belmont S, Muñoz B, Zúñiga P, Pereira J, Mezzano D: High prevalence of bleeders of unknown cause among patients with inherited mucocutaneous bleeding. A prospective study of 280 patients and 299 controls. Haematologica 2007, 92(3):357-365.

43. Israels SJ, El-Ekiaby M, Quiroga T, Mezzano D: Inherited disorders of platelet function and challenges to diagnosis of mucocutaneous bleeding. Haemophilia : the official journal of the World Federation of Hemophilia 2010, 16 Suppl 5:152-159.

44. Gresele P, Harrison P, Bury L, Falcinelli E, Gachet C, Hayward CP, Kenny D, Mezzano D, Mumford AD, Nugent $D$ et al: Diagnosis of suspected inherited platelet function disorders: results of a worldwide survey. Journal of thrombosis and haemostasis : JTH 2014, 12(9):1562-1569.

45. Gresele P, Bury L, Mezzasoma AM, Falcinelli E: Platelet function assays in diagnosis: an update. Expert 
Rev Hematol 2019, 12(1):29-46.

46. Gresele P: Diagnosis of inherited platelet function disorders: guidance from the SSC of the ISTH. Journal of thrombosis and haemostasis : JTH 2015, 13(2):314-322.

47. Navred K, Martin M, Ekdahl L, Zetterberg E, Andersson NG, Strandberg K, Norstrom E: A simplified flow cytometric method for detection of inherited platelet disorders-A comparison to the gold standard light transmission aggregometry. PloS one 2019, 14(1):e0211130.

48. Boknas N, Ramstrom S, Faxalv L, Lindahl TL: Flow cytometry-based platelet function testing is predictive of symptom burden in a cohort of bleeders. Platelets 2018, 29(5):512-519.

49. Gum PA, Kottke-Marchant K, Poggio ED, Gurm H, Welsh PA, Brooks L, Sapp SK, Topol EJ: Profile and prevalence of aspirin resistance in patients with cardiovascular disease. The American journal of cardiology 2001, 88(3):230-235.

50. Hankey GJ, Eikelboom JW: Aspirin resistance. Lancet (London, England) 2006, 367(9510):606-617.

51. Nguyen TA, Diodati JG, Pharand C: Resistance to clopidogrel: a review of the evidence. Journal of the American College of Cardiology 2005, 45(8):1157-1164.

52. Müller I, Besta F, Schulz C, Massberg S, Schönig A, Gawaz M: Prevalence of clopidogrel nonresponders among patients with stable angina pectoris scheduled for elective coronary stent placement. Thrombosis and haemostasis 2003, 89(5):783-787.

53. Le Quellec S, Bordet JC, Negrier C, Dargaud Y: Comparison of current platelet functional tests for the assessment of aspirin and clopidogrel response. A review of the literature. Thrombosis and haemostasis 2016, 116(4):638-650.

54. Cuisset T, Frere C, Quilici J, Barbou F, Morange PE, Hovasse T, Bonnet JL, Alessi MC: High posttreatment platelet reactivity identified low-responders to dual antiplatelet therapy at increased risk of recurrent cardiovascular events after stenting for acute coronary syndrome. Journal of thrombosis and haemostasis : JTH 2006, 4(3):542-549.

55. Hulstein JJ, de Groot PG, Silence K, Veyradier A, Fijnheer R, Lenting PJ: A novel nanobody that detects the gain-of-function phenotype of von Willebrand factor in ADAMTS13 deficiency and von Willebrand disease type 2B. Blood 2005, 106(9):3035-3042.

56. Hulstein JJ, van Runnard Heimel PJ, Franx A, Lenting PJ, Bruinse HW, Silence K, de Groot PG, Fijnheer R: Acute activation of the endothelium results in increased levels of active von Willebrand factor in hemolysis, elevated liver enzymes and low platelets (HELLP) syndrome. Journal of thrombosis and haemostasis : JTH 2006, 4(12):2569-2575.

57. de Mast Q, Groot E, Lenting PJ, de Groot PG, McCall M, Sauerwein RW, Fijnheer R, van der Ven A: Thrombocytopenia and release of activated von Willebrand Factor during early Plasmodium falciparum malaria. The Journal of infectious diseases 2007, 196(4):622-628.

58. Chen SF, Xia ZL, Han JJ, Wang YT, Wang JY, Pan SD, Wu YP, Zhang B, Li GY, Du JW et al: Increased active von Willebrand factor during disease development in the aging diabetic patient population. Age (Dordrecht, Netherlands) 2013, 35(1):171-177.

59. Hyseni A, Kemperman H, de Lange DW, Kesecioglu J, de Groot PG, Roest M: Active von Willebrand factor predicts 28-day mortality in patients with systemic inflammatory response syndrome. Blood 2014, 123(14):2153-2156. 
60. Rutten B, Maseri A, Cianflone D, Laricchia A, Cristell NA, Durante A, Spartera M, Ancona F, Limite L, Hu D et al: Plasma levels of active Von Willebrand factor are increased in patients with first ST-segment elevation myocardial infarction: a multicenter and multiethnic study. European heart journal Acute cardiovascular care 2015, 4(1):64-74.

61. van der Vorm LN, Visser R, Huskens D, Veninga A, Adams DL, Remijn JA, Hemker HC, Rensma PL, van Horssen R, de Laat B: Circulating active von Willebrand factor levels are increased in chronic kidney disease and end-stage renal disease. Clinical kidney journal 2020, 13(1):72-74.

62. van der Vorm LN, Huskens D, Kicken CH, Remijn JA, Roest M, de Laat B, Miszta A: Effects of Repeated Bouts of Exercise on the Hemostatic System. Seminars in thrombosis and hemostasis 2018, 44(8):710722.

63. van der Vorm LN, Li L, Huskens D, Hulstein JJJ, Roest M, de Groot PG, Ten Cate H, de Laat B, Remijn JA, Simons SO: Acute exacerbations of COPD are associated with a prothrombotic state through platelet-monocyte complexes, endothelial activation and increased thrombin generation. Respiratory medicine 2020, 171:106094. 


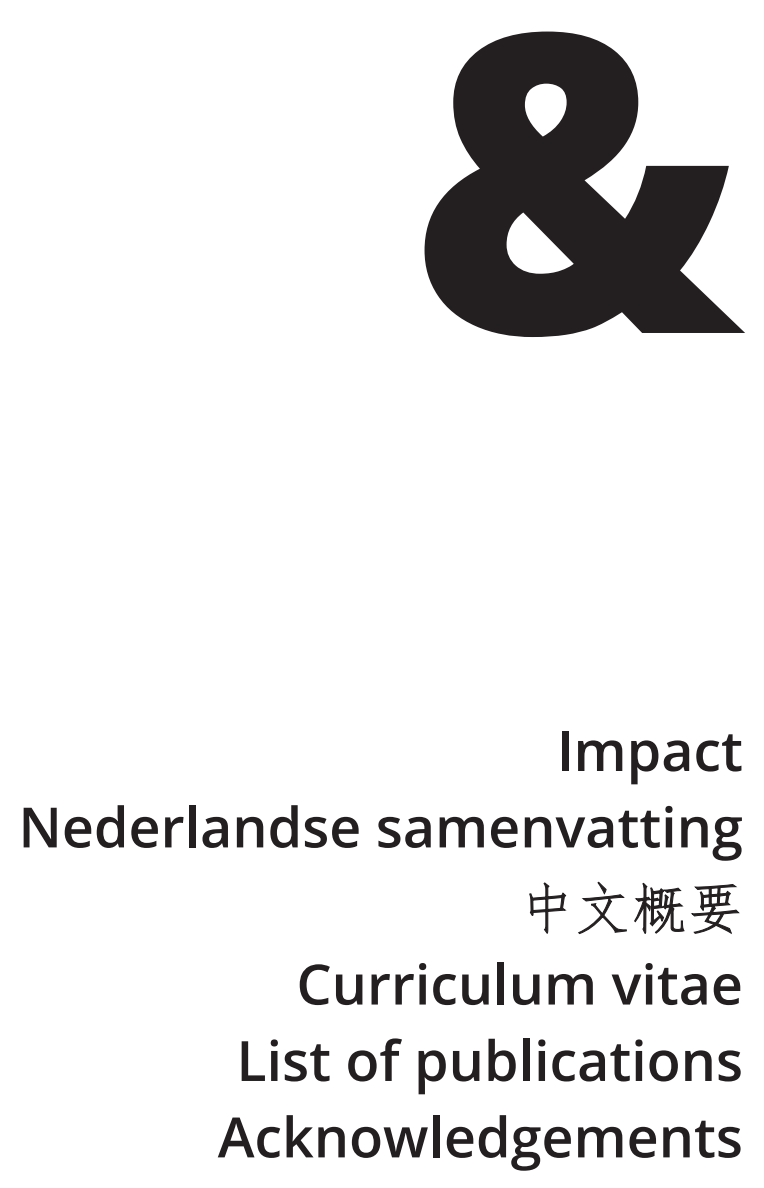


\section{IMPACT}

Platelet activation and coagulation are essential parts in hemostasis. Many steps in platelet thrombus formation are closely connected to the different stages of thrombin formation. We explored a novel mechanism for the interplay between platelets and coagulation by studying the FXII-independent initiation of thrombin generation by collagen in vitro. More in detail, platelets activated with GPVI agonists (CRP-XL and convulxin), but not with a2 31 agonists (GFOGER), were able to induce thrombin generation. The FXa generation experiments done with purified coagulation factors and inhibition of an anti-FIX antibody, along with the recovery of TG in FIX deficient plasma with the addition of FVIII, suggested FIXa activity from GPVI activated platelets. Taken together, our findings indicate that apart from the well-known participations of platelets in coagulation (providing an assembly site for coagulation factors by exposed PS, releasing partially activated FV and polyP), activated platelets can contribute to thrombin generation via FIXa activity. The clinical significance of our findings remains to be demonstrated. Our findings show that coagulation can be initiated via tissue factor and contact activation independent triggers. This pathway may be important for certain forms of venous thrombo-embolism (VTE), because in the majority of VTE patients, there are no indications of exposure to TF or contact activation. Furthermore, our findings indicate a potential mechanism to explain why the clinical manifestations in haemophilia B patients are milder compared to the bleeding severity in haemophilia A patients [1], while treatment of haemophilia B or A mice with platelet-targeted FIX or FVIII gene therapy, respectively, reduces bleeding and restores haemostasis [2, 3].

On-treatment multiple myeloma (MM) patients have an increased incidence of venous thromboembolism (VTE), despite a high prevalence of thrombocytopenia and anaemia. We investigated platelet reactivity, platelet activation, plasma thrombin generation and whole blood thrombin generation profiles in MM patients and healthy controls. Compared to controls, plasma thrombin generation was not different in treated MM patients. However, they showed diminished platelet reactivity, delayed initiation but increased thrombin generation potential in whole blood, which were associated with several properties of RBCs and platelets. These findings help to understand the disbalanced haemostasis in treated MM patients with an emphasis on the contributions of blood cells, which may further help providing more specific prevention strategies for VTE in MM patients. Also, the WB-TG test applied in this study may be a promising tool for future research studying blood cells in coagulation and thrombosis related diseases. COPD is a chronic, progressive condition with a high prevalence. The Global Burden of Disease Study 2015 estimated the global prevalence of COPD at about 174 million cases [4]. For COPD patients, the periodic deteriorations called exacerbations pose the highest financial burden due to supplementary treatment and increased hospital 
admissions [5]. Also, exacerbations are associated with an increased risk of thrombotic cardiovascular events, which contribute significantly to the overall disease morbidity and mortality [6]. Exacerbations were found to be associated with increased PMCs, but not with changes in platelet reactivity. VWF and TG were significantly higher during AE-COPD compared to convalescence. Furthermore, PMCS, VWF and TG were positively associated with systemic inflammation. Therefore, we concluded that acute exacerbations are associated with an inflammation-associated prothrombotic state. Our findings justify the further investigation on biomarkers of endothelial cell activation, platelet pathophysiology, hyper-coagulation and hypo-fibrinolysis to predict the risk of exacerbation relapse and of cardiovascular events. If large clinical outcome studies would prove that these markers have additional diagnostic value, implementation in clinical chemistry laboratories may be considered.

In the secondary prophylaxis of CVD such as myocardial infarction and stroke, antiplatelet therapy is the cornerstone. High on-treatment residual platelet reactivity (HRPR) is associated with high risk of thrombotic events and thus testing response to anti-platelet therapy may bring value for patients [7]. LTA is the standard technique to monitor HRPR, however, LTA is poorly standardized despite existing guidelines, requires a considerable volume of fresh blood and is time and labor intensive. We found a moderate agreement between MesADP-triggered whole blood platelet activation test (WB-PACT) and ADP-triggered LTA on recognizing HRPR in patients receiving aspirin and clopidogrel. This study indicates that WB-PACT is an optional platelet reactivity test for monitoring anti-platelet therapy. Patients with a high platelet reactivity on clopidogrel/ aspirin treatment are suitable for treatment with new antiplatelet agents like prasugrel or ticagrelor.

The prevalence of inherited platelet function disorders (IPFDs) among the general population has not been established, and little is known about the burden of IPFDs, probably because the identification of inherited platelet function defects is challenging due to the complexity of platelet function and consequently, the large diversity of platelet defects. It is relatively straightforward to diagnose severe IPFDs since these patients with distinct clinical and laboratory features can readily be detected with current available diagnostic tools, while for mild IPFDs the diagnosing could be cumbersome due to the heterogeneous phenotype that requires a combination of tests. We investigated the correlation between the flow cytometric WB-PACT and LTA in patients with suspected bleeding diathesis for platelet function defect identification. A moderate correlation was found between LTA and WB-PACT and there was agreement between both tests in 62\% of the cases. By comparing to bleeding score, the WB-PACT detected more abnormal responses than LTA in high BS patients. Therefore, with the advantages of less handling steps, suitability for low platelet count samples and neonatal blood samples, WB-PACT 
may have added value for the routine diagnostic work-up in platelet function defect recognition. Accurate and timely diagnosis for patients with suspected IPFDs would help them with appropriate treatments, minimize the associated bleeding risk, and therefore improve their quality of life [8].

Lastly, an immunosorbent assay was characterized to directly detect circulating VWF in its active conformation. Current evidence indicated a possible role of active VWF measurements in monitoring TTP patients, while large, clinical outcome studies are still needed for exploring its clinical usefulness [9, 10]. At the moment, the value of active VWF quantification seems to lie in the research field for unraveling the pathophysiological mechanisms of hemostatic complications in a variety of conditions [11-13].

\section{Concluding remark}

The research presented in this thesis provides new insights in the initiation of coagulation and in the role of blood cells in coagulation. Both insights may lead to new diagnostic targets to estimate the risk of thrombosis in high-risk patients. Furthermore, intervention in platelet activation induced thrombin generation and in cell dependent thrombin generation may be novel targets for anti-thrombotic treatment. These hypotheses need to be further explored in prospective follow-up studies and, if confirmed, in intervention studies. 


\section{REFERENCES}

1. Castaman G, Matino D: Hemophilia A and B: molecular and clinical similarities and differences. Haematologica 2019, 104(9):1702-1709.

2. Li B, Wu Z, Xu W, Han W, Liu J, Wang D, Zhang G: Treatment of a Hemophilia B Mouse Model with Platelet-Targeted Expression of Factor IX Padua. Hum Gene Ther 2021, 32(9-10):506-516.

3. Cai Y, Shi Q: Platelet-Targeted FVIII Gene Therapy Restores Hemostasis and Induces Immune Tolerance for Hemophilia A. Frontiers in Immunology 2020, 11(964).

4. Global, regional, and national incidence, prevalence, and years lived with disability for 310 diseases and injuries, 1990-2015: a systematic analysis for the Global Burden of Disease Study 2015. Lancet (London, England) 2016, 388(10053):1545-1602.

5. Sullivan SD, Ramsey SD, Lee TA: The economic burden of COPD. Chest 2000, 117(2 Suppl):5s-9s.

6. Anthonisen NR, Connett JE, Enright PL, Manfreda J: Hospitalizations and mortality in the Lung Health Study. American journal of respiratory and critical care medicine 2002, 166(3):333-339.

7. Cuisset T, Frere C, Quilici J, Barbou F, Morange PE, Hovasse T, Bonnet JL, Alessi MC: High posttreatment platelet reactivity identified low-responders to dual antiplatelet therapy at increased risk of recurrent cardiovascular events after stenting for acute coronary syndrome. Journal of thrombosis and haemostasis : JTH 2006, 4(3):542-549.

8. Gresele P, Falcinelli E, Bury L: Laboratory diagnosis of clinically relevant platelet function disorders. International journal of laboratory hematology 2018, 40 Suppl 1:34-45.

9. Peyvandi F, Scully M, Kremer Hovinga JA, Knöbl P, Cataland S, De Beuf K, Callewaert F, De Winter H, Zeldin RK: Caplacizumab reduces the frequency of major thromboembolic events, exacerbations and death in patients with acquired thrombotic thrombocytopenic purpura. Journal of thrombosis and haemostasis : JTH 2017, 15(7):1448-1452.

10. Scully M, Cataland SR, Peyvandi F, Coppo P, Knöbl P, Kremer Hovinga JA, Metjian A, de la Rubia J, Pavenski K, Callewaert F et al: Caplacizumab Treatment for Acquired Thrombotic Thrombocytopenic Purpura. The New England journal of medicine 2019, 380(4):335-346.

11. Hulstein JJ, Lenting PJ, de Laat B, Derksen RH, Fijnheer R, de Groot PG: beta2-Glycoprotein I inhibits von Willebrand factor dependent platelet adhesion and aggregation. Blood 2007, 110(5):1483-1491.

12. de Mast Q, Groot E, Asih PB, Syafruddin D, Oosting M, Sebastian S, Ferwerda B, Netea MG, de Groot PG, van der Ven AJ et al: ADAMTS13 deficiency with elevated levels of ultra-large and active von Willebrand factor in P. falciparum and P. vivax malaria. The American journal of tropical medicine and hygiene 2009, 80(3):492-498.

13. Djamiatun K, van der Ven AJ, de Groot PG, Faradz SM, Hapsari D, Dolmans WM, Sebastian S, Fijnheer R, de Mast Q: Severe dengue is associated with consumption of von Willebrand factor and its cleaving enzyme ADAMTS-13. PLoS neglected tropical diseases 2012, 6(5):e1628. 


\section{NEDERLANDSE SAMENVATTING}

Dit proefschrift richt zich op de wisselwerking tussen bloedplaatjes en stolling en op het gebruik van flowcytometrische plaatjesfunctie testen in een klinische omgeving.

Het belang van de complexe interactie tussen bloedplaatjes en stolling werd jaren onderschat. Hoewel er aandacht besteed wordt aan het feit dat veel stappen binnen de vorming van een bloedplaatjes trombus nauw gelinkt zijn met de verschillende stadia van trombinevorming, is er nog weinig bekend over de mechanismes die hieraan ten grondslag liggen. Zo kan collageen bijvoorbeeld stolling tot stand brengen via de activatie van FXII, maar ook onafhankelijk van FXII. In hoofdstuk 2 hebben we een nieuw mechanisme voor initiatie van de stolling onderzocht dat gebaseerd is op bloedplaatjesactivatie door collageen. Niet alleen collageen maar ook andere GPVI-agonisten (CRP-XL en convulxin) geactiveerde bloedplaatjes bleken trombinevorming te induceren. Trombinegeneratie experimenten met remmers of met factor deficiënte plasma's suggereerden dat deze initiatie van bloedplaatjesactivering via GPVI niet afhankelijk is van de stollingsfactoren FXII, FXI of TF/FVIIa. Met behulp van een gezuiverd systeem waar FXa vorming werd gemeten in de aan en afwezigheid van anti-FIX antilichamen alsook met trominevorming experimenten in FIX deficiënt plasma gesupplementeerd met FVIII werd aangetoond dat GPVI-geactiveerde plaatjes FIXa activiteit leveren.

Hoewel de wisselwerking tussen bloedplaatjes en het stollingssysteem belangrijk is en veel ziekte- en therapiegerelateerde trombotische gebeurtenissen worden veroorzaakt door beschadigde of aangetaste bloedplaatjes in plaats van door veranderingen in stollingsfactoren, zijn er nog steeds geen toegankelijke instrumenten om dit in klinisch onderzoek te bestuderen. Visco-elastische testen zoals ROTEM en TEG worden gebruikt om bloedcelgemedieerde trombotische risico's te bestuderen. Deze testen zijn echter niet specifiek genoeg om als alternatief voor plasmacoagulatietesten of bloedplaatjesfunctietesten te dienen. Onze groep heeft onlangs een volbloedtrombinegeneratietest (WB-TG) ontwikkeld die een goede correlatie vertoont met de trombinegeneratietest in plasma.

Bij patiënten met multipel myeloom (MM) die onder behandeling zijn werd aangetoond dat ze een verhoogde incidentie van VTE hebben ondanks hoge prevalentie van trombocytopenie en anemie. In hoofdstuk 3 hebben we in MM patiënten ne als in gezonde donoren trombinegeneratie profielen gemeten in plasma en in volbloed. Verder werd ook de bloedplaatjesreactiviteit en bloedplaatjesactiviatie gemeten. In vergelijking met de controles werd verminderde bloedplaatjesreactiviteit waargenomen bij patiënten terwijl het niveau van bloedplaatjesacitvatie, aangegeven door de hoeveelheid plaatjes-monocyt complexen, niet significant verschilde tussen de groepen. 
Opmerkelijk was de bevinding dat in volbloed de vorming van trombine in patiënten vertraagd van start ging, maar dat er een verhoogde trombine potentiaal gemeten werd terwijl er geen verschillen werden gemeten tussen patiënten en controles in plasma trombinegeneratie. De verschillen en volbloed trombinegeneratie waren geassocieerd met eigenschappen van rode bloedcellen en bloedplaatjes. Deze bevindingen helpen om de onevenwichtige hemostase bij MM patiënten te begrijpen.

In hoofdstuk 4 werd de protrombotische toestand bij patiënten met chronische obstructieve longziekte (COPD) na acute exacerbatie onderzocht. COPD-patiënten hebben een verhoogd risico op cardiovasculaire voorvallen, vooral na acute exacerbaties. Exacerbaties gaan gepaard met verhoogde systemische ontsteking die stolling kan veroorzaken. In deze prospectieve cohortstudie werd bepaald hoe acute exacerbaties bij COPD de bloedplaatjesactivatie, het endotheel, de stolling en fibrinolyse beïnvloeden. Ook de associatie met ontsteking werd onderzocht aangezien bekend is dat de prevalentie van trombotische voorvallen toeneemt tijdens en kort na acute exacerbaties van COPD (AE-COPD). Het exacte mechanisme is onbekend. Allereerst vonden we dat bloedplaatjesactivatie, gemeten als de vorming van bloedplaatjes-monocyte complexen, verhoogd was tijdens AE-COPD. Aan de andere kant was de reactiviteit van de bloedplaatjes niet significant verschillen met de tijdstippen dag 3 van behandeling en herstel. In deze patiënten vertoonden zowel bloedplaatjesactivatie als reactiviteit associaties met systemische ontsteking (CRP niveau). Tijdens AE-COPD werd ook een verhoogde trombinevorming en een verhoogde concentratie VWF (zowel antigeen als de actieve vorm) gemeten wat samen een protrombotische toestand suggereert. Deze studie toont aan dat de gemeten parameters mogelijk biomarkers kunnen zijn voor het voorspellen van acute exacerbaties en cardiovasculaire gebeurtenissen.

In het tweede deel van dit proefschrift ligt de focus op de volbloed flowcytometrische bloedplaatjesfunctietest (WB-PACT). In hoofdstuk 5 hebben we de mogelijke toepassing ervan bestudeerd voor het monitoren van de effecten van antibloedplaatjesmedicatie. In vitro Dubbele antibloedplaatjestherapie (DAPT) met aspirine en de P2Y 12 receptorantagonist clopidogrel wordt vaak voorgeschreven als secundaire preventie voor patiënten na percutane angioplastiek met stentimplantatie en voor patiënten met atherosclerotische cardiovasculaire ziekte. Verschillende studies wezen op een hoge restbloedplaatjesreactiviteit (HRPR) tijdens de behandeling met DAPT met een hoger risico op trombotische voorvallen. Om HRPR te detecteren werd in deze studie de bloedplaatjesreactiviteit gemeten met behulp van de WB-PACT en vergeleken met de LTA. Een matige overeenkomst werd gevonden tussen MeSADP-gestimuleerde WBPACT en de ADP-gestimuleerde LTA en onze data suggereerden dat de WB-PACT een mogelijk alternatief kan zijn voor LTA in het monitoren van DAPT. 
In hoofdstuk 6 werd de bloedplaatjesfunctie getest met behulp van WB-PACT in een patiëntenpopulatie met een bloedingsdiathese. Hoewel LTA de 'gouden standaard' van de bloedplaatjesfunctietesten is, is deze techniek nog niet gevoelig genoeg voor het detecteren van lichte bloedplaatjesfunctiedefecten en kan deze techniek ook niet altijd het risico op bloedingscomplicaties in patiënten met verschillende bloedplaatjesdefecten voorspellen. Daarom is er een dringende nood aan alternatieve methoden om aanvullende informatie te geven over milde funtiestoornissen van de bloedplaatjes. In deze studie werden WB-PACT gestimuleerd met meerdere agonisten vergeleken met LTA. Voor het detecteren van VWF-disfunctie werd de VWF-functietoepassing van de WB-PACT vergeleken met de door ristocetine geïnduceerde agglutinatiemeting. Beide technieken werden met elkaar vergeleken op basis van een scoringssysteem en een matige correlatie werd gevonden. Bij patiënten met een verhoogde bloedingsscore was de WB-PACT beter in het detecteren van verminderde plaatjesreactiviteit dan de LTA. Bovendien herkende de WB-PACT een aantal patiënten met afwijkingen in VWFconcentratie of -functie. Als we hoofdstuk 5 en 6 samen beschouwen kan WB-PACT een toegevoegde waarde hebben voor de routinematige diagnostiek bij het monitoren van de bloedplaatjesfunctie.

In hoofdstuk 7 werd een ELISA gekarakteriseerd om circulerend VWF in zijn actieve conformatie te detecteren. Deze test is gebaseerd op een recombinant, van lama afgeleid, antilichaam gericht tegen een cryptisch epitoop in het A1 domein van VWF. Naast de karakterisatie van de analytische prestaties van deze test werden ook positieve correlaties met andere gevestigde VWF methoden alsook met de VWF-functietoepassing van de WB-PACT vastgesteld. Tenslotte werden voor actief VWF en voor andere VWF parameters referentie-intervallen bepaald in een populatie van 120 gezonde vrijwilligers. Concluderend beschrijft dit proefschrift nieuwe inzichten in bloedplaatjesactivatie gemedieerde initiatie van stolling. De studies in dit proefschrift bevestigen dat bloedcellen cruciale spelers zijn in de regulatie van stolling, vooral in pathologische ziektes. 


\section{中文概要}

本论文的重点是血小板和凝血之间的相互作用以及流式细胞仪血小板功能检测在临床中的 应用。

多年来, 血小板和凝血之间复杂相互作用的重要性一直被低估。尽管已经注意到血小板血 栓形成的许多步骤与凝血酶形成的不同阶段密切相关, 但对其潜在机制知之甚少。例如, 胶原蛋白可以通过激活 FXII 介导凝血, 但也可以独立于 FXII。在第 2 章中, 我们探索 了一种基于胶原蛋白激活血小板的新型凝血启动机制。不仅胶原蛋白而且其他 GPVI 激动 剂（CRP-XL 和 convulxin）激活的血小板也被发现诱导凝血酶形成。使用抑制剂或缺乏 因子的血浆进行的凝血酶生成实验表明, 通过 GPVI 启动血小板活化的凝血酶形成不依赖 于凝血因子 FXII、FXI 或 TF/FVIIa。使用纯化的系统, 其中在存在和不存在抗 FIX 抗体 的情况下测量 FXa 形成, 以及在补充有 FVIII 的FIX 缺陷血浆中的凝血酶生成实验, 显 示 GPVI 激活的血小板提供 FIXa 活性。

尽管血小板和凝血系统之间的相互作用很重要, 并且许多与疾病和治疗相关的血栓事件是 由受损的血小板而不是凝血因子的变化引起的, 但在临床研究中仍然没有可用的工具来研 究这一点。ROTEM 和 TEG 等粘弹性测定用于研究血细胞介导的血栓形成风险。然而, 这 些测试的特异性不足以替代血浆凝固测试或血小板功能测试。我们小组最近开发了一种全 血凝血酶生成测试 (WB-TG)，它与血浆凝血酶生成测试显示出良好的相关性。

尽管血小板减少症和贫血症的发病率很高，但接受治疗的多发性骨髓瘤（MM）患者的 VTE 发生率增加。在第 3 章中, 我们测量了 MM 患者以及健康供体的血浆和全血中的凝血酶 生成谱。此外, 还测量了血小板反应性和血小板活化程度。与对照组相比, 在患者中观察 到血小板反应性降低, 而血小板活化水平（由血小板一单核细胞复合物的数量表示）在组 间没有显着差异。值得注意的是，在全血中，患者凝血酶形成的开始被延迟，但测量到的 凝血酶生成潜力增加，而患者和对照之间在血浆凝血酶生成方面没有测量到差异。全血凝 血酶生成的差异与红细胞和血小板一些特征有关。这些发现有助于了解 MM 患者的不平衡 的止血状态。

第4章对慢性阻塞性肺病患者急性加重期 (AECOPD) 的促血栓形成状态进行了研究。COPD 患者发生心血管事件的风险增加, 尤其是在急性加重后, 这与可导致凝血的全身炎症增加 有关。这项前瞻性队列研究旨在了解 COPD 的急性加重如何影响血小板活化、内皮、凝血 和纤溶。同时还研究了这些与炎症的关联, 因为已有文献报道慢阻肺患者急性加重期间和 之后不久血栓形成事件的发生率会增加，但确切的机制仍然未知。首先，我们发现血小板 活化（以血小板一单核细胞复合物的形成来衡量）在AECOPD 期间增加。另一方面，血小板 反应性在患者接受治疗和恢复的第 3 天没有显著差异。在研究的患者中, 血小板活化水 平和反应性均显示与全身炎症 ( CRP 水平) 相关。同时在 $\mathrm{AE}-\mathrm{COPD}$ 期间, 血浆凝血酶形 成增加, VWF (抗原和活性形式) 浓度增加, 这些都暗示存在血栓前状态。这些结果提示 血小板活化水平可能是预测急性加重和心血管事件的生物标志物。 
在本论文的第二部分, 重点是全血流式细胞仪血小板功能测试（WB-PACT）。在第 5 章 中, 我们研究了其在监测抗血小板药物作用方面的潜在应用。阿司匹林和 P 2 Y 12 受体拮抗 剂氯吡格雷的体外双重抗血小板治疗 (DAPT) 通常作为动脉䉼样硬化性心脏病患者经皮血 管成形术和支架植入术后的二级预防。几项研究表明, 在 DAPT 治疗期间存在高残留血小 板反应性 (HRPR), 血栓事件风险较高。为了检测 HRPR, 本研究中使用 WB-PACT 测量血 小板反应性并与 LTA 进行比较。在 MeSADP激动的 WB-PACT 和 ADP 激动的 LTA 之间 发现了中等一致性, 我们的数据表明 WB-PACT 可能是 LTA 监测 DAPT 的潜在替代方案。 第 6 章使用 WB-PACT 在具有潜在出血风险的患者群体中测试了血小板功能。虽然 LTA 是 血小板功能检测的 “金标准” , 但该技术的灵敏度尚不足以检测轻度血小板功能缺陷, 也 不能很好地预测各种血小板缺陷患者发生出血并发症的风险。因此, 迫切需要替代方法来 提供有关轻度血小板功能障碍的额外信息。在这项研究中, 与 LTA 相比, WB-PACT 受到 多种激动剂的刺激。为了检测 VWF 功能障碍, 将 WB-PACT 的 VWF 功能应用与瑞斯托霉素 诱导的凝集测量进行了比较。在评分系统的基础上对这两种技术进行了比较, 发现了适度 的相关性。在出血评分升高的患者中, WB-PACT 在检测血小板反应性降低方面优于 LTA。 此外, WB-PACT 还识别出许多 VWF 浓度或功能异常的患者。如果我们将第 5 章和第 6 章 放在一起考虑, WB-PACT 可能对监测血小板功能的常规诊断具有附加价值。

第 7 章对检测血液中活性VWF的 ELISA方法进行了表征。该测定是基于针对 VWF A1 域中 的隐蔽表位的重组美洲羊驼衍生抗体。除了表征该测试的分析性能外, 还发现了与其他已 建立的 VWF 检测方法以及与 WB-PACT 的 VWF 功能应用有正相关。最后, 本研究确定了 120 名健康志愿者的活化 VWF水平和其他 VWF相关参数的参考区间。

综上, 本论文描述了血小板活化介导的凝血启动的新见解, 提示血细胞是凝血调节的关键 参与者, 同时也研究了全血流式细胞仪血小板功能测试未来可能的应用方向。 


\section{CURRICULUM VITAE}

Li Li was born on December $13^{\text {th }}, 1992$ in China. She started her medical study in 2009 and finished in 2017 with a bachelor and a master degree. The same year she was funded by a scholarship from China Scholarship Council to begin PhD training in Maastricht University and Synapse Research Institute, under the supervision of Prof. dr. Hugo ten cate, Dr. Dana Huskens and Dr. Mark Roest. During her PhD, she worked on the interaction between platelets and coagulation and on the use of flow cytometric platelet function testing in clinical settings. 


\section{LIST OF PUBLICATIONS}

Huskens D\#, Li L\#, Florin L, de Kesel P, de Laat B, Roest M, Devreese KMJ: Flow cytometric analysis of platelet function to improve the recognition of thrombocytopathy. Thromb Res 2020, 194:183-189. (\#co-first author)

van der Vorm LN, Li L, Huskens D, Hulstein JJ, Roest M, de Groot PG, Ten Cate H, de Laat B, Remijn JA, Simons SO: Acute exacerbations of COPD are associated with a prothrombotic state through platelet-monocyte complexes, endothelial activation and increased thrombin generation. Respiratory medicine 2020, 171:106094.

van der Vorm LN, Li L, Huskens D, Chayouâ W, Kelchtermans H, de Groot PG, Roest M, Remijn JA, de Laat B: Analytical characterization and reference interval of an enzyme-linked immunosorbent assay for active von Willebrand factor. PloS one 2019, 14(2):e0211961.

Huskens D\#, Li L\#, Florin L, de Kesel P, de Laat B, Roest M, Devreese KMJ: Flow cytometric analysis of platelet function to detect high on-treatment residual platelet reactivity in patients on dual antiplatelet therapy period ((\#co-first author, accepted by International Journal of Laboratory Hematology)

Li L, Roest M, Meijers JCM, de Laat B, Urbanus RT, de Groot PG, Huskens D. Platelet activation via glycoprotein $\mathrm{VI}$ initiates thrombin generation: a potential role for platelet-derived factor IX? (Submitted)

Li L, Roest M, Sang Y, Remijn J, Fijnheer R, Smit K, Huskens D, Wan J, de Laat B, Konings J. Patients with multiple myeloma have a disbalanced whole blood thrombin generation profile. (Submitted)

Sang Y, Remijn J, Fijnheer R, Smit K, Korporaal S, Urbanus RT, Huskens D, Joke Konings J, Li L, de Laat B, Roest M. High Prevalence of Acquired Platelet Secretion Defects in Multiple Myeloma. (submitted)

\section{PRESENTATIONS AT CONFERENCES}

2020 September, Platelet society ECR virtual symposium, Li L. Platelet activation via GPVI initiates FXII- and TF-independent thrombin generation. Oral presentation

2020 July, International Society on Thrombosis and Haemostasis (ISTH) annual congress,

Li L. Platelet activation via GPVI initiates FXII- and TF-independent thrombin generation. Poster presentation

2019 May, EAS (European Atherosclerosis Society) congress, Li L. Platelet activation by collagen can initiate coagulation, independent of factor XII and tissue factor. Poster presentation 


\section{ACKNOWLEDGEMENTS}

I received lots of support and help in the past four years. Without these, this thesis would not be possible and I would like to express my sincere gratitude.

I would like to thank members of the assessment committee, Prof. dr. Yvonne Henskens, Prof. em. Johan Heemskerk, Dr. Rory Koenen, Prof. dr. Ton Lisman, Prof. dr. Monique, thank you for taking your time to review this thesis and giving suggestions!

My promotion team has made great efforts to help me with our scientific output and this thesis. I would like to thank my promotor Prof. dr. Hugo ten Cate, for giving me the opportunity to finish my PhD with you! Thank you for your help on my extension and revising my thesis! Dear Dana, I am grateful for your help through my PhD, thank you for sparing time showing me lab skills, discussing protocols, revising manuscripts, thesis and giving advices on my presentations! I admire your efficiency and being critical in research, you can always spot the errors that I didn't see. I remember the colourful flags you hung up in the lab the first year I came and keep the small bull reminder. I very much appreciate your understanding and support in the last period of my PhD! Dear Mark, thank you for setting challenges in the past years and sparing time discussing my results, revising my manuscripts and thesis! I very much appreciate your help in the last period of my PhD! Though we sometimes have different opinions, they let me reconsider my thoughts and what I am not good at as well as what I might be suitable for.

I want to acknowledge help from all present and previous colleagues at Synapse! Dear Dr. Bas de Laat, thank you for offering help when I was in trouble and being supportive and flexible for my way to finish PhD! Dear Prof. dr Philip G. de Groot, I am grateful for your input on CRP project, I learned a lot from the meetings with you! Dear Joke, you are always calm and nice to work with, thank you for your help on TIMM project and the manuscript! Dear Lisa, it's a nice opportunity for me to work with you on the reference study and COPD study! Dear Eric, Tessa, Rob and Tea, thank you for offering the purified proteins, inhibitors and your help on my experiments! Dear Hilde, thank you for keeping the lab organized, drawing blood and encouraging us! Dear Iris and Caroline, thank you for handling my documents, ordering reagents and the nice talks! Dear Marisa and Romy, thank you for your daily help in the lab! Dear Adam, thank you for showing me plasmin generation! Dear Cecile, Saartje and Suzzane, thank you for your help on blood drawing! Dear Martijn, Walid and Eartha, nice chats with you in the past years! 翠翠师 姐, 佩服你的淡定从容, 谢谢你给我实验上的建议和生活上的帮助, 和你讨论时总是很有 启发, 希望你和Armando开心幸福, 小馒头健康成长! 亚秋师姐, 你是我在荷兰相处时间 最多的人, 在你身边总是觉得安稳, 佩服你的平和包容, 为别人着想, 万分感激在马城最 后的日子里你的帮助, 祝你在美国一切顺利, 我们国内见! 万俊师哥珊珊师姐, 冬梅师姐 军哥, 谢谢你们在学习生活方面的各种经验, 邀请我去你们家聚餐, 祝你们工作顺利生活 
幸福！秋婷, 黄盛世, 思雨和邹金幂, 谢谢你们在实验室的帮助, 祝你们毕业顺利! I want to acknowledge help from our collaborators! I want to thank Prof. dr Joost C.M. Meijers and Dr. Rolf T. Urbanus for providing crucial reagents and insights for CRP project from which I learned a lot! I also want to thank Dr. Rob Fijnheer, Dr. Jasper A. Remijn, Dr. Karel Smit, Mark, Bianca and other people in the clinical chemistry lab of Meander Medical center for your help in TIMM study! Lastly, I want to thank Prof. dr. Katrien M. J. Devreese and Lisa Florin for offering insightful suggestions and collecting data on WB-PACT Ghent project!

I want to thank Maike for supporting me on the platelet-monocyte complexes project! I also want to thank Oriona, Daphne, Nikita, Yves, Maikel, Melanie, Hilde, Harmen and many other previous interns, for the joy you brought!

I want to thank everyone who donated blood for the experiments in this thesis!

Friends in Maastricht and Friends back in China, thank you for the support you provided and the good times we had together! 谢谢王老师在我硕士阶段的包容和一直以来的关心 和鼓励！曼莉, 和你总是有很多可以聊, 在过去的一年, 你对我的帮助太多我感激不尽, 祝答辩顺利, 找到心仪的职位! 刘豫, 开心在你家聊天吃饭, 祝你回国工作顺利! 湘兰, 和你还有师姐一起出游的时光真是美好, 谢谢一路的陪伴! 于奎, 香香, 雅文, Claudia, 若汐, 张永刚, 王萍、张明师姐, 谢谢在马城时你们的帮助! 望, 夏夏, 在你们面前我还 是那个我, 谢谢你们给我信心! 蕾蕾, 珊珊, 冰洁, 朱兰, 谢谢这些年来的帮助!

Finally, I want to extend my gratitude to my family! 阿公阿婆, 爷爷奶奶, 妈, 否磊, 玲玲, 亮亮, 镇利, 老舅舅妈, 大伯姑妈们, 大阿姨姨夫, 二阿姨姨夫, 谢谢有你们在! 
